\author{
Universidade de São Paulo \\ Instituto de Física
}

\title{
Efeitos do movimento coletivo na evaporação e fissão nuclear
}

\author{
Motahareh Abbasi Shanbehbazari
}

Orientador: Prof. Dr. Airton Deppman

Coorientadora: Profa. Dra. Gayane Karapetyan

Tese de doutorado apresentada ao Instituto de Física da Universidade de São Paulo, como requisito parcial para a obtenção do título de Doutora em Ciências.

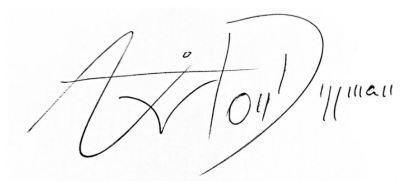

Banca Examinadora:

Prof. Dr. Airton Deppman - Orientador (IFUSP)

Prof. Dr. Luiz Carlos Chamon (IFUSP)

Prof. Dr. Adimir dos Santos (IPEN)

Prof. Dr. Pedro Carlos Russo Rossi (UFABC)

Prof. Dr. Arturo Rodolfo Samana (UESC) 


\section{FICHA CATALOGRÁFICA}

\section{Preparada pelo Serviço de Biblioteca e Informação}

do Instituto de Física da Universidade de São Paulo

Abbasi Shanbehbazari, Motahareh

Efeitos do movimento coletivo na evaporação e fissão nuclear. São Paulo, 2021.

Tese (Doutorado) - Universidade de São Paulo. Instituto de Física. Depto. de Física Experimental

Orientador: Prof. Dr. Airton Deppman

Área de Concentração: Física Nuclear

Unitermos: 1. Reação nuclear; 2. Método de Monte Carlo; 3. Fissão nuclear. 


\title{
University of São Paulo
}

Physics Institute

\section{Effects of Collective Motion on Nuclear Evaporation and Fission}

\author{
Motahareh Abbasi Shanbehbazari
}

Supervisor: Prof. Dr. Airton Deppman

Co-supervisor: Profa. Dra. Gayane Karapetyan

Thesis submitted to the Physics Institute of the

University of São Paulo in partial fulfillment of the requirements for the degree of Doctor of Science.

Examining Committee:

Prof. Dr. Airton Deppman - Advisor (IFUSP)

Prof. Dr. Luiz Carlos Chamon (IFUSP)

Prof. Dr. Adimir dos Santos (IPEN)

Prof. Dr. Pedro Carlos Russo Rossi (UFABC)

Prof. Dr. Arturo Rodolfo Samana (UESC) 


\section{Acknowledgment}

First and foremost I cannot but express my sincere gratitude to my advisor, Prof. Dr. Airton Deppman, for his continuing support and encouragement, inspiration, patience, and invaluable help and thoughtful advices he provided in developing my knowledge in Physics. It is an honor for me to be his student.

I am very thankful as well to my husband, Hamed Panjeh, for his constant encouragement throughout my study period and patiently supporting me at every stage of my research and standing behind me.

I would like to thank my colleagues and friends, Ramon Perez Varona and Evandro Oliveira Andrade Segundo for their help in my dissertation.

I thank profusely Prof. Dr. Luiz Carlos Chamon, Prof. Dr. Adimir dos Santos, Prof. Dr. Pedro Carlos Russo Rossi and Prof. Dr. Arturo Rodolfo Samana for serving on my $\mathrm{PhD}$ committee and their invaluable advices.

I deeply appreciate Prof. Dr. Arnold Gammal, Prof. Dr. Sergio Jose Barbosa Duarte and Prof. Dr. Luiz Carlos Chamon for their invaluable comments in my qualification exam.

I would like to thank the Coordenação de Aperfeiçoamento de Pessoal de Nível Superior - Brasil (CAPES), which supported this work through Finance Code 001.

Last but not least, I would like to express my gratitude to all the staff at graduate committee (CPG), specially Andrea Wirkus and Eber De Patto Lima, for their kind help and co-operation in this period. 
University of São Paulo

\section{Abstract}

Physics Institute

PhD student in Sciences

Study of heavy-ion-induced fusion-fission reactions is important for understanding the physics of nuclear matter and nuclear models. In this work, we study the mechanism of nuclear reactions of ${ }^{11} \mathrm{~B}+{ }^{181} \mathrm{Ta},{ }^{11} \mathrm{~B}+{ }^{197} \mathrm{Au},{ }^{11} \mathrm{~B}+{ }^{209} \mathrm{Bi}$ and ${ }^{7} \mathrm{Li}+{ }^{208} \mathrm{~Pb}$ at intermediate energy using CRISP model and also analyze the nuclear reaction processes starting from the initial nucleus-nucleus collision up to the fragments production. The experimental data are measured at the LNR Phasotron (JINR, Dubna, Russia) and also taken from Ref. [1]. The fission process is studied using multimodal fission model for ${ }^{7} \mathrm{Li}+{ }^{208} \mathrm{~Pb}$ at $245 \mathrm{MeV},{ }^{11} B+{ }^{209} \mathrm{Bi}$ at $146 \mathrm{MeV}$ and ${ }^{11} B+{ }^{197} A u$ at $137.5 \mathrm{MeV}$ energy, and pure symmetric distribution for ${ }^{11} B+{ }^{181} \mathrm{Ta}$ at 245.4 and $125.7 \mathrm{MeV}$ and ${ }^{11} B+{ }^{197} A u$ at 255.5 $\mathrm{MeV}$ energy. A new branch of CRISP model has been developed to describe heavy-ion induced reactions by implementation of the Rotating Finite Range model. Experimental mass-yield distributions are compared with the results of the Monte Carlo simulation code CRISP on the framework of the Random Neck Rupture model. The experimental data of spallation products are also described by the CRISP model. The fission cross sections, fissility and fission-fragment mass distributions are calculated and compared with experimental data. We conclude that CRISP model provides a good description of intermediate energy heavy-ion-induced fission.

Keywords: CRISP model; heavy-ion-induced fission; nucleus-nucleus collision; Rotating Finite Range model; Random Neck Rupture model 
Universidade de São Paulo

\section{Resumo}

Instituto de Física

Doutorando em Ciências

O estudo de reações de fusão-fissão induzidas por íons pesados é importante para intender a origem da matéria nuclear e de modelos nucleares. Neste trabalho, estudamos o mecanismo de reações nucleares de ${ }^{11} \mathrm{~B}+{ }^{181} \mathrm{Ta},{ }^{11} \mathrm{~B}+{ }^{197} \mathrm{Au},{ }^{11} \mathrm{~B}+{ }^{209} \mathrm{Bi} \mathrm{e}^{7} \mathrm{Li}+{ }^{208} \mathrm{~Pb}$ na energia intermediária usando o modelo CRISP e também analisamos os processos de reação nuclear a partir de colisão inicial de núcleo-núcleo até a produção dos fragmentos de reação. Os dados experimentais são medidos no LHR Phasotron (JINR, Dubná, Rússia) e tambem de Ref. [1]. O processo de fissão é estudado usando o modelo de fissão multimodal para ${ }^{7} \mathrm{Li}+{ }^{208} \mathrm{~Pb}$ na $245 \mathrm{MeV},{ }^{11} \mathrm{~B}+{ }^{209} \mathrm{Bi}$ na $146 \mathrm{MeV}$ e ${ }^{11} B+{ }^{197} A u$ na energia $137.5 \mathrm{MeV}$ e a distribuição simétrica pura para as reações de ${ }^{11} B+{ }^{181} \mathrm{Ta}$ na 245.4 e $125.7 \mathrm{MeV} \mathrm{e}{ }^{11} B+{ }^{197} A u$ na energia $255.5 \mathrm{MeV}$ foi considerada. Para descrever as reações induzidas por íons pesados, um ramo novo de modelo CRISP foi desenvolvida através da implementação de um modelo Rotating Finite Range. As distribuições de massa dos fragmentos experimentais são comparadas com os resultados do código de simulação de Monte Carlo, CRISP, na padrão do modelo Random Neck Rupture. Os dados experimentais de produtos de espalação também são descritos pelo modelo CRISP. As seções de choque dos fragmentos de fissão, a fissilidade e as distribuições de massa dos fragmentos são calculadas e comparadas com os dados experimentais. Concluímos que o modelo CRISP fornece uma boa descrição de fissão induzida por íons pesados á energia intermediária.

Palavras-chaves: modelo CRISP; fissão induzida por íons pesados; colisão de núcleonúcleo; modelo Rotating Finite Range; modelo Random Neck Rupture 


\section{Contents}

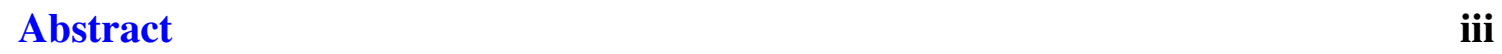

Resumo iv

List of Figures viii

List of Tables $\quad$ xii

1 Introduction 1

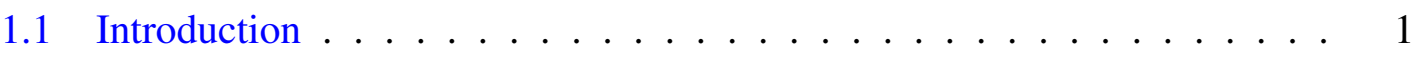

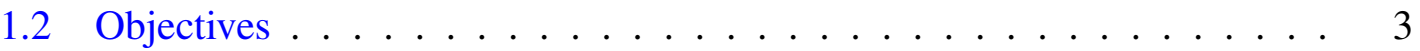

2 Nuclear Models 5

2.1 Liquid drop model $\ldots \ldots \ldots \ldots$. . . . . . . . . . . 5

2.2 Fermi Gas model . . . . . . . . . . . . . . . . 6

2.3 Shell model . . . . . . . . . . . . . . . . . . . . . 7

2.4 Collective model . . . . . . . . . . . . . . . . . . 8

2.5 Nilsson model . . . . . . . . . . . . . . . . . . . . . . . . 10

2.6 Myers-Swiatecki model . . . . . . . . . . . . . . . . . . . 13

2.7 Brosa model . . . . . . . . . . . . . . . . . . . . . . 16

2.7.1 Shape parameters .................. 17

2.7.2 A real flat neck representation (prescission shape) . . . . . . 18

2.7.3 The embedded spheroids . . . . . . . . . . . . . . . . . 18

2.7.4 Fundamentals of random neck rupture . . . . . . . . . . . . . 19

2.7.4.1 Scission as a sequence of instabilities . . . . . . . 22 
2.7.5 Standard, superlong and supershort . . . . . . . . . . 22

2.7.5.1 Cayley tree of nuclear fission . . . . . . . . . . 24

3 CRISP Model 2

3.1 MCMC model . . . . . . . . . . . . . . . . . . . . . 26

3.2 MCEF model . . . . . . . . . . . . . . . . . . . . . . . 27

3.3 Multi-modal fission . . . . . . . . . . . . . . . . . 30

3.4 Additions and improvements of CRISP model . . . . . . . . . . . . . . 34

3.4.1 Correction in MCEF (evaporation width ratios) . . . . . . . . . 34

3.4.1.1 Level density parameters in the old formulas . . . . . 35

3.4.1.2 Level density parameters in the new formulas . . . . 35

3.4.2 Implementation of heavy-ion reactions $\ldots \ldots . \ldots 38$

3.4.2.1 Ion Event Generator . . . . . . . . . . . . . 38

3.4.2.2 Angular-momentum-dependent fission barrier . . . . 38

4 Heavy-ion-induced nuclear reactions $\quad 39$

4.1 Nucleus-nucleus collision model in CRISP . . . . . . . . . . . . . . . . . 39

4.1.1 Initial conditions and point of contact . . . . . . . . . . 39

4.1.2 Nucleonic transference from projectile to target . . . . . . . . . 42

4.1.3 Peripheral collisions .................. 43

4.2 Excitation energy and angular momentum . . . . . . . . . . . 43

4.3 Angular momentum in heavy-ion-induced nuclear reactions . . . . . . . . 44

4.3.1 Rotating liquid drop model (RLDM) . . . . . . . . . . . . . . 45

4.3.2 Rotating finite range model $(\mathrm{RFRM}) \ldots \ldots . \ldots 46$

4.3.2.1 Extension of RFRM with Nix model . . . . . . . . 48

5 Results and Discussion $\quad 50$

5.1 Mass-yield distribution $\ldots \ldots \ldots \ldots$. . . . . . . . . . . . 50

5.2 Pre- and post-scission nucleons . . . . . . . . . . . . . . . 57

5.3 Fissility parameter ....................... 61

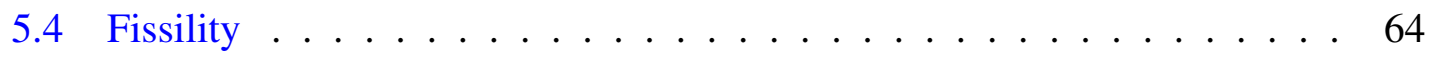

5.4.1 Possible mechanisms to the formation of IMFs . . . . . . . . 66 
6 Conclusion

7 Appendix 72

$7.1 \quad$ Appendix A . . . . . . . . . . . . . . 72

Bibliography 


\section{List of Figures}

2.1 Nuclear square wells for neutrons and protons. The well parameters are adjusted to give the observed binding energy $B^{\prime}[12] \ldots \ldots 6$

2.2 Three-dimensional harmonic oscillator and its energy levels [12]. . . . . 8

2.3 Splitting of the states with a given value of $l$ into two states. The spin-orbit interaction depresses the state with total angular momentum $j=l+\frac{1}{2}$ and raises the one with $j=l-\frac{1}{2}[12] \ldots \ldots \ldots . \ldots \ldots$

2.4 Approximate level pattern for nucleons. The number of nucleons in each level and the cumulative totals are shown. Neutrons and protons have essentially the same level pattern up to 50. From then on, some deviations

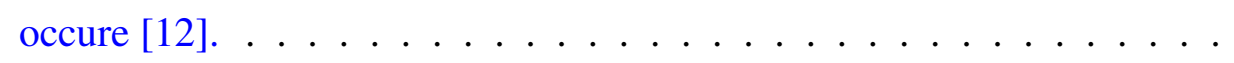

2.5 The more realistic potential resembling the actual nuclear density distribution is replaced by a harmonic oscillator potential or a square well [12].

2.6 Level diagram in the Nilsson model. Each state can accept two nucleons

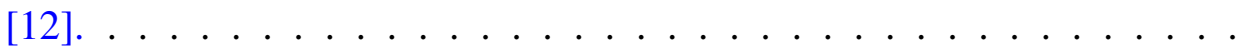

2.7 The top part of the figure shows schematically how a negative shell correction leads to a spherical shape with special stability, whereas a positive shell correction leads to a deformed equilibrium shape. In the bottom left-hand part, the amplitude of the shell-correction bump along the valley of stability is shown. When the amplitude exceeds a certain critical value, indicated by the dashed line, deformed equilibrium shapes appear. The shell correction is a consequence of the bunching of levels into bands corresponding to magic numbers, as shown schematically on the right [18]. 14 
2.8 This is a plot of the revised shell damping function which replaces the Gaussian function in the original mass formula. Its equation is $(1-$ $\left.2 \theta^{2}\right) e^{-\theta^{2}}$, where $\theta$ is a deformation variable [18] . . . . . . . . 14

2.9 The deformation energy is illustrated when the new shell correction is included. In (a) shell correction for the sphere is positive, and this leads to a deformed equilibrium shape whose mass sags below the liquid drop value. In (b) the case of a magic nucleus is illustrated, where a secondary deformed equilibrium shape is predicted [18] . . . . . . . . . . . 15

2.10 Visualization of the degrees of freedom $l, r, z, c$ and $s$. The surface depicted is a special Lawrencian shape displayed in the coordinates $\rho$ and $\zeta$ [20] . . . . . . . . . . . . . . . . . . 17

2.11 The flat-neck representation (Eq. 2.18), upper part; and the embedded spheroids (Eqs. 2.19 and 2.20), lower part. Note the different origin of the coordinates $\rho$ and $\zeta$ compared to that in Fig. 2.10 [20]. . . . . . . . .

2.12 Some important correlations that a prescission shape mediates. Shown are mass yields $Y$ (dotted lines, right-hand-side scales) and neutron multiplicities $\bar{\nu}$ (solid lines, left-hand-side scales) as functions of fragment mass number A. Part (a) shows a supershort prescission shape and its products, part (b) the symmetric, part (c) the standard and part (d) the superasymmetrical prescission shape [20] . . . . . . . . . . . . . 20

2.13 Random neck rupture and the sawtooth shape of neutron multiplicity $\bar{\nu}(A)$

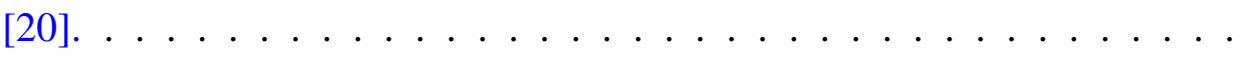

2.14 Channel probabilities $p_{c}$ as it varies with the size of fissioning system. This is a schematic picture. We have no precise method to compute the channel weights $p_{c}[20] . \ldots \ldots \ldots \ldots$

2.15 Cayley tree of nuclear fission. Emphasized are distinguished points of the potential energy as minima $\circ$, barriers $\times$, bifurcation points $\bullet$, prescission shapes + and their connections (heavy lines) $[20] \ldots \ldots$. . . . . . .

3.1 Comparing the results of the spallation for a fixed atomic number $Z=88$ using the old formulas in CRISP and the corrected formulas. . . . . . . 
3.2 Comparing the results of the fission for a fixed atomic number $Z=90$ using the old formulas in CRISP and the corrected formulas. Both results are identical. . . . . . . . . . . . . . . . . . . 37

4.1 System of coordinates used to describe the nucleus-nucleus collisions in CRISP [56] . . . . . . . . . . . . . . . . . . . . 40

4.2 General predictions of fission barriers for different angular momenta [54]. 45

5.1 Mass-yield distributions for the reaction of ${ }^{11} \mathrm{~B}$ ions with ${ }^{181} \mathrm{Ta}$ target at 245.4 and $125.7 \mathrm{MeV}$ energy. The blue solid line indicates the results of the fission cross sections as a function of fragment mass $A$ and the red dotted line corresponds to the spallation cross section, both calculated by the CRISP code, (a) and (c) without any modification in CRISP with the fission barrier at $L=0$, and (b) and (d) after inserting RFRM and Nix models. The experimental data (solid squares) are taken from Ref. [1] . . .

5.2 Mass-yield distributions for the reaction of ${ }^{11} B$ ions with ${ }^{197} A u$ target at 255.5 and $137.5 \mathrm{MeV}$ energy. The blue solid line indicates the results of the fission cross sections as a function of fragment mass $A$ and the red dotted line corresponds to the spallation cross section, both calculated by the CRISP code, (a) and (c) without any modification in CRISP with the fission barrier at $L=0$, and (b) and (d) after inserting RFRM and Nix models. . . . . . . . . . . . . . . . . . .

5.3 Mass-yield distributions for the reaction of ${ }^{11} \mathrm{~B}$ ions with ${ }^{209} \mathrm{Bi}$ target at $146 \mathrm{MeV}$ energy and the reaction of ${ }^{7} \mathrm{Li}$ ions with ${ }^{208} \mathrm{~Pb}$ target at 245 $\mathrm{MeV}$ energy. The blue solid line indicates the results of the fission cross sections as a function of fragment mass $A$ and the red dotted line corresponds to the spallation cross section, both calculated by the CRISP code, (a) and (c) without any modification in CRISP with the fission barrier at $L=0$, and (b) and (d) after inserting RFRM and Nix models. The experimental data (solid squares) for the reaction of ${ }^{11} \mathrm{~B}+{ }^{209} \mathrm{Bi}$ at $146 \mathrm{MeV}$ is taken from Ref. $[1] \ldots \ldots \ldots \ldots$. . . . . . . . . . . . 53 
5.4 Color map distribution of the fissility parameter $Z_{f}^{2} / A_{f}$ for the fissioning systems of the reactions ${ }^{11} \mathrm{~B}+{ }^{181} \mathrm{Ta}$ at 245.4 and $125.7 \mathrm{MeV}$ energies, ${ }^{11} \mathrm{~B}+{ }^{197} \mathrm{Au}$ at 255.5 and $137.5 \mathrm{MeV},{ }^{7} \mathrm{Li}+{ }^{208} \mathrm{~Pb}$ at $245 \mathrm{MeV}$ and ${ }^{11} B+{ }^{209} \mathrm{Bi}$ at $146 \mathrm{MeV}$. The solid red line corresponds to the critical fissility parameter as a function of $Z_{f}[81,82] \ldots \ldots$. . . . . . . 63

5.5 The same as the right column of Figs. 5.1 and 5.3. The black dash-dotted line corresponds to the results of the Gaussian fit to the IMF experimental data and the black dashed line is obtained by summing contribution from this mechanism to spallation distribution and indicates the addition of IMF cross section to the counterpart pair of products in spallation region. 69 


\section{List of Tables}

3.1 Values of the relevant parameters in Dostrovsky's empirical formulas. . . 28

3.2 Parameters used to determine the calculated charge distribution. . . . . . 32

5.1 Parameter values of Eq. (3.13) used to determine experimental and calculated mass distributions; and fission $\left(\sigma_{f}\right)$ and total $\left(\sigma_{t o t}\right)$ cross sections of ${ }^{11} \mathrm{~B}+{ }^{181} \mathrm{Ta}$ reaction at 245.4 and $125.7 \mathrm{MeV}$ energies. . . . . . . . . 55

5.2 Parameter values of Eq. (3.13) used to determine experimental and calculated mass distributions; and fission $\left(\sigma_{f}\right)$ and total $\left(\sigma_{t o t}\right)$ cross sections of ${ }^{11} B+{ }^{197} A u$ reaction at 255.5 and $137.5 \mathrm{MeV}$ energies. . . . . . . . . . 56

5.3 Parameter values of Eq. (3.13) used to determine experimental and calculated mass distributions; and fission $\left(\sigma_{f}\right)$ and total $\left(\sigma_{t o t}\right)$ cross sections of ${ }^{7} \mathrm{Li}+{ }^{208} \mathrm{~Pb}$ reaction at $245 \mathrm{MeV}$ and ${ }^{11} \mathrm{~B}+{ }^{209} \mathrm{Bi}$ reaction at $146 \mathrm{MeV}$ energy. .......................... 56

5.4 Mean mass number $\left(\bar{A}_{S}\right)$; mean mass of the fissioning nucleus $\left(\bar{A}_{f}\right)$ after evaporation of pre-scission nucleons from the compound nucleus; mean mass of the summation of binary-fission fragments $\left(\bar{A}_{f f}\right)$ after evaporation of post-scission nucleons from the fragments; average number of prescission neutrons $\left(\nu_{\text {pre_n }}\right)$, pre-scission protons $\left(\nu_{\text {pre }-p}\right)$, post-scission neutrons $\left(\nu_{\text {post_n }}\right)$, and post-scission protons $\left(\nu_{\text {post_p }}\right)$; and the average number of total emitted nucleons $\left(\nu_{T}\right)$ of ${ }^{11} B+{ }^{181} \mathrm{Ta}$ reaction at 245.4 and 125.7 $\mathrm{MeV}$ energies. . . . . . . . . . . . . . . . . . 58 
5.5 Mean mass number $\left(\bar{A}_{S}\right)$; mean mass of the fissioning nucleus $\left(\bar{A}_{f}\right)$ after evaporation of pre-scission nucleons from the compound nucleus; mean mass of the summation of binary-fission fragments $\left(\bar{A}_{f f}\right)$ after evaporation of post-scission nucleons from the fragments; average number of prescission neutrons $\left(\nu_{\text {pre_n }}\right)$, pre-scission protons $\left(\nu_{\text {pre_p }}\right)$, post-scission neutrons $\left(\nu_{\text {post_n }}\right)$, and post-scission protons $\left(\nu_{\text {post_p }}\right)$; and the average number of total emitted nucleons $\left(\nu_{T}\right)$ of ${ }^{11} B+{ }^{197} A u$ reaction at 255.5 and 137.5 $\mathrm{MeV}$ energies. . . . . . . . . . . . . . . . . . 59

5.6 Mean mass number $\left(\bar{A}_{S}\right)$; mean mass of the fissioning nucleus $\left(\bar{A}_{f}\right)$ after evaporation of pre-scission nucleons from the compound nucleus; mean mass of the summation of binary-fission fragments $\left(\bar{A}_{f f}\right)$ after evaporation of post-scission nucleons from the fragments; average number of prescission neutrons $\left(\nu_{\text {pre_n }}\right)$, pre-scission protons $\left(\nu_{\text {pre_p }}\right)$, post-scission neutrons $\left(\nu_{\text {post_n }}\right)$, and post-scission protons $\left(\nu_{\text {post_p }}\right)$; and the average number of total emitted nucleons $\left(\nu_{T}\right)$ of ${ }^{7} \mathrm{Li}+{ }^{208} \mathrm{~Pb}$ reaction at $245 \mathrm{MeV}$ and ${ }^{11} \mathrm{~B}+{ }^{209} \mathrm{Bi}$ reaction at $146 \mathrm{MeV}$ energy. . . . . . . . . . . . .

5.7 Charge and mass number of the fissioning system and the average fissility parameter $\left(Z_{f}^{2} / A_{f}\right)$ calculated by CRISP for the ${ }^{11} B+{ }^{181} \mathrm{Ta}$ reaction at 245.4 and 125.7 MeV energies, ${ }^{11} B+{ }^{197} A u$ reaction at 255.5 and 137.5 $\mathrm{MeV},{ }^{7} \mathrm{Li}+{ }^{208} \mathrm{~Pb}$ reaction at $245 \mathrm{MeV}$ and ${ }^{11} \mathrm{~B}+{ }^{209} \mathrm{Bi}$ reaction at 146 $\mathrm{MeV}$ energy. The critical fissility parameter $\left(Z_{f}^{2} / A_{f}\right)_{c r}$. is also presented.

5.8 Fissility of the ${ }^{11} \mathrm{~B}+{ }^{181} \mathrm{Ta}$ and $p+{ }^{181} \mathrm{Ta}$ reactions at 245.4 and 125.7 MeV energies, ${ }^{11} B+{ }^{197} A u$ and $p+{ }^{197} A u$ reactions at 255.5 and 137.5 $\mathrm{MeV}$ energies, ${ }^{7} \mathrm{Li}+{ }^{208} \mathrm{~Pb}$ reaction at $245 \mathrm{MeV}$ and the fission probability of the ${ }^{11} B+{ }^{209} B i$ and $p+{ }^{209} B i$ reactions at $146 \mathrm{MeV}$. . . . . . . . 65

5.9 Parameters used to determine the distribution of the light fragment mass. . 68 


\section{Chapter 1}

\section{Introduction}

\subsection{Introduction}

Although nuclear fission was discovered about eighty years ago (in 1938), it has continued to pose challenges in the last eight decades $[2,3,4]$. One of these challenges is heavy-ioninduced fission reactions at intermediate energies which gives us a deep insight into the angular momentum transferred in nucleus-nucleus collisions and the lowering of fission barriers with increasing angular momentum. While many investigations have been done on actinide nuclei with different projectiles in a wide range of energy, the study of heavyion-induced fission on pre-actinide targets is limited.

Among the whole processes of nucleus-nucleus interaction, fission represents the most interesting phenomenon. Fission is a slow process in the nuclear time-scale, involving deformation of the whole nucleus. Studies of the mass and charge distributions of the fission fragments at intermediate energies provide important information about the dynamics of the reaction. Cross sections determination for the interaction of charged particles and heavy ions with nuclei reveals that the reaction mechanism for compound system formation varies with incident particle energy. The dependence of experimental data of fission at medium excitation energies shows that at the time of determination of the charge, mass and excitation energy, the angular momentum of the fissioning nucleus transferred in the entrance channel of the reaction plays an essential role. Thus, the process of scission and formation of fragments is influenced not only by the temperature, but also by the total angular momentum of the fissioning nucleus. 
While fission of actinides has been investigated in details using different projectiles as photons, protons, and heavy-ions in the large energetic scale, the study of pre-actinide nuclei is limited. In the present work, for the first time, a systematic analysis of heavy-ioninduced reaction and its fission fragment production is modeled with a developed branch of Monte Carlo CRISP (acronym for Collaboration Rio-Ilhéus-São Paulo) [5] code with multimodal fission model to describe heavy-ion-induced fission on ${ }^{197} \mathrm{Au},{ }^{181} \mathrm{Ta},{ }^{208} \mathrm{~Pb}$, and ${ }^{209} \mathrm{Bi}$ targets at intermediate energies. Heavy-ion-induced fission has been studied by the recently developed Monte Carlo CRISP code extended to include the rotating liquid drop model.

Compared to CRISP model, the new version of the Liege intra-nuclear cascade model (INCL++) $[6,7]$ is an equivalent code which has also been recently redesigned and now supports heavy-ion projectiles up to mass $18[8,9,10]$. CRISP and INCL++ models have many similarities, such as implementation of multi-collisional approach, Pauli blocking and conservation of energy and momentum after each event. Besides, the extension of the INCL++ to heavy-ion induced reactions needed the preparation of projectile and target nuclei and to take into account the binding of the projectile nucleus which are described in details in ref. [6], and the method of implementing nucleus-nucleus collision in CRISP model is also discussed in Chapter 4.

The comparison of CRISP calculations with the experimental mass distribution of the fragments provides information about the fission process and the mechanism of fragments formation. Also, the probability of symmetric and asymmetric fission is investigated using the so-called fissility parameter, defined as $Z^{2} / A$. Besides, since heavy-ion projectiles transfer a significant angular momentum to the system, compared to the nucleon-nucleus collisions, in this study, the effect of imparted angular momentum on the fission barrier and the fissility has been studied.

It is important to observe that low energy fission is determined not only by the properties of the bulk of nuclear matter as described by mass models or similar approaches, but also the shell effects play an important role. As the excitation energy of the fissioning nucleus increases, those shell effects decrease [11]. For the problem studied in the present work, shell effects may appear, for instance, in the formation of asymmetric mass fission fragments, and the effects are summarized by the parameters related to the mass 
asymmetry. It will be shown here that for the present study, the shell effects are negligible since the mass fragment distributions are mostly symmetric.

The Monte Carlo method used in CRISP model consists of the simulation of real situations from the generation of random numbers and allows to simulate very complex problems that involve the transport of particles through nuclear matter. Nuclear reactions present a very favorable field of study for the application of Monte Carlo techniques, with two fundamental approaches to their simulation, intranuclear cascade and evaporationfission competition depending mainly on the degrees of freedom involved and the energy of the reaction.

The first step of reaction is described by an intranuclear cascade where all the nucleons of the nucleus are treated as independent particles, and their movements are considered in straight lines and each two of them interact with each other when they reach the relative distances of the order of nuclear force interaction. When this stage ends, the nucleus reaches a state of thermodynamic equilibrium and can be described by macroscopic variables, such as temperature and density of levels. From the nucleus mass number, atomic energy, excitation energy and angular momentum, one can obtain a good statistical description of the system and can obtain expressions for the calculation of its particle emission and nuclear fission widths.

\subsection{Objectives}

The main objective of this thesis is to study intermediate nucleus-nucleus reactions using CRISP model. For this purpose, the following works have been done:

- An improvement in some formulas in Monte Carlo for Evaportion-Fission (MCEF) stage,

- Study of heavy-ion-induced fusion-fission processes,

- Study of the effects associated with transferred angular momentum at the analysis of fission induced by the accelerated heavy ions

- Study of different models presented to simulate nucleus-nucleus collisions, 
- Implementation of Rotating Finite Range model (RFRM) into CRISP model

- Convertion of BARFIT Fortran 77 subroutine to $\mathrm{C}++$ language and making it compatible with CRISP code

- Insertion of BARFIT into CRISP code to acquire the correct angular-momentumdependent fission barrier,

- The results of CRISP are compared with experimental data. 


\section{Chapter 2}

\section{Nuclear Models}

Nuclear models used in CRISP are briefly described here.

\subsection{Liquid drop model}

One of the most striking facts about nuclei is the "approximate constancy of nuclear density": the volume of a nucleus is proportional to the number of A of constituents [12].

The same fact holds for liquids, and one of the early nuclear models introduced by Bohr and Weizsacker was patterned after liquid drops; "nuclei are considered to be incompressible liquid droplets of extremely high density."

The liquid drop model leads to an understanding of the trend of binding energies with atomic number, and it also gives a physical picture of fission process. Consider a nucleus consisting of $A$ nucleons, $Z$ protons, and $N$ neutrons. The total mass of such a nucleus is somewhat smaller than the sum of the masses of its components because of the binding energy $B$ which holds the nucleons together.

$$
\frac{B}{c^{2}}=Z m_{p}+N m_{n}-m_{\text {nuclear }}(Z, N) .
$$

Bethe-Weizsacker relation for binding energy of a nucleus $(A, N)$ is:

$$
B=a_{v} A-a_{s} A^{\frac{2}{3}}-a_{s y s} \frac{(Z-N)^{2}}{A}-a_{c} \frac{Z^{2}}{A^{\frac{1}{3}}} .
$$

A typical set of constants (determined by fitting the experimentally observed binding 
energies) is:

$$
\begin{array}{ll}
a_{v}=15.6 \mathrm{MeV}, & a_{s}=16.8 \mathrm{MeV}, \\
a_{\text {sys }}=23.3 \mathrm{MeV}, & a_{c}=0.72 \mathrm{MeV} .
\end{array}
$$

\subsection{Fermi Gas model}

The semi-empirical binding-energy relation obtained in the previous section is based on treating the nucleus like a liquid drop. Such an analogy is an oversimplification, and the nucleus has many properties that can be explained more simply in terms of independentparticle behavior rather than in terms of the strong-interaction picture implied by the liquid drop model.

The most primitive independent-particle model is obtained "if the nucleus is treated as a degenerate Fermi gas of nucleons". The nucleons are assumed to move freely, except for effects of exclusion principle, throughout a sphere of radius $R=R_{0} A^{1 / 3}, R_{0} \approx 1.2$ fm. The situation is represented in Fig. 2.1 by two wells, one for neutrons and one for protons.

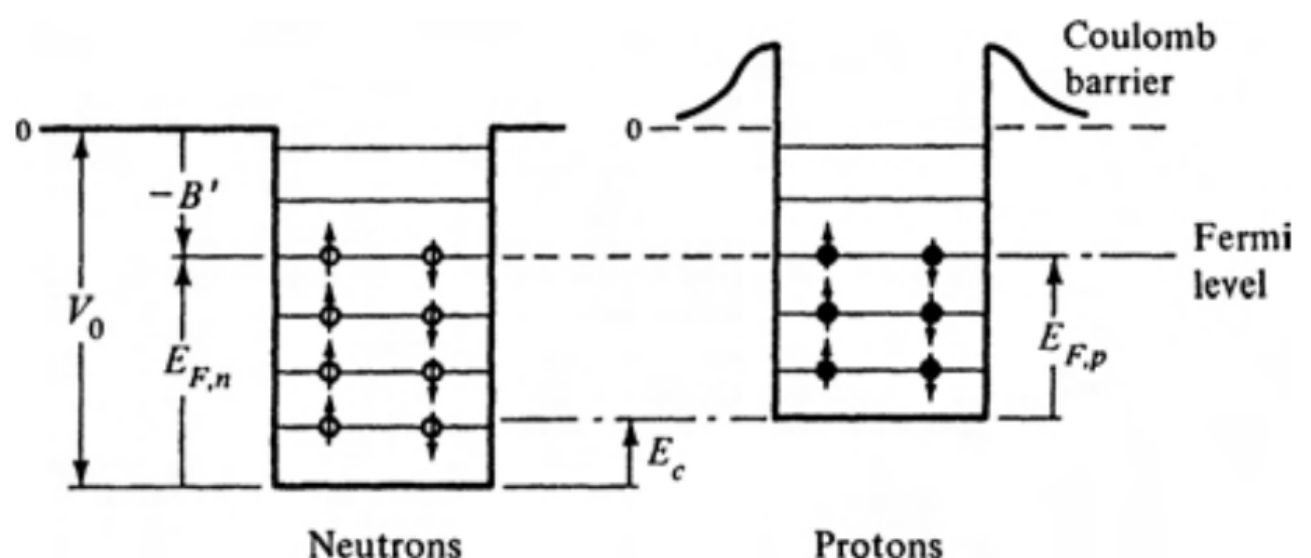

Figure 2.1: Nuclear square wells for neutrons and protons. The well parameters are adjusted to give the observed binding energy $B^{\prime}[12]$.

Free neutrons and free protons, far away from the wells, have the same energy, and the zero levels for the two wells are the same. The two wells, however, have different shapes and different depths because of the Coulomb energy:

$$
E_{c}=\frac{3}{5} \frac{(Z e)^{2}}{R}
$$


The bottom of the proton well is higher than the bottom of the neutron well by an amount $E_{c}$, and the proton potential has a Coulomb barrier. Protons that try to enter the nucleus from the outside are repelled by the positive charge of the nucleus; they must either "tunnel" through the barrier or have enough energy to pass over it.

The wells contain a finite number of levels. Each level can be occupied by two nucleons, one with spin up and one with spin down. It is assumed that, under normal conditions, the nuclear temperature is so low that the nucleons occupy the lowest states available to them. Such a situation is described by the term degenerate Fermi gas [13].

"Fermi gas model" considers the nucleus as a collection of non-interacting fermions whose density is fixed somehow from the outside. The purpose of this model is to clarify which of the nuclear properties is due just to the "Pauli exclusion principle" at the given density, and how much of these properties remain to be explained by the detailed dynamics of the interacting nucleons.

\subsection{Shell model}

The liquid drop and the Fermi gas models cannot explain specific properties of excited nuclear states. Nuclei display particularly stable configurations if $Z$ or $N$ (or both) is one of the "magic numbers" [12]:

$2,8,20,28,50,82,126$.

Magic numbers say that some kind of shells exist in nuclei. Nuclides with either $Z$ or $N$ equal to one of the magic numbers $2,8,20,28,50,82,126$ are "particularly stable".

Consider first the harmonic oscillator whose energy levels are shown in Fig. 2.2. The group of degenerate levels corresponding to one particular value of $N$ is called an oscillator shell. The degeneracy of each shell is given by equation: $\frac{1}{2}(N+1)(N+2)$. In the application to nuclei, each level can be occupied by two nucleons, and consequently the degeneracy is given by $(N+1)(N+2)$.

A nucleon with orbital angular momentum $l$ can be in two states, with total angular momenta $l \pm \frac{1}{2}$ (Fig. 2.3). The splitting between states $j=l+\frac{1}{2}$ and $j=l-\frac{1}{2}$ is caused 


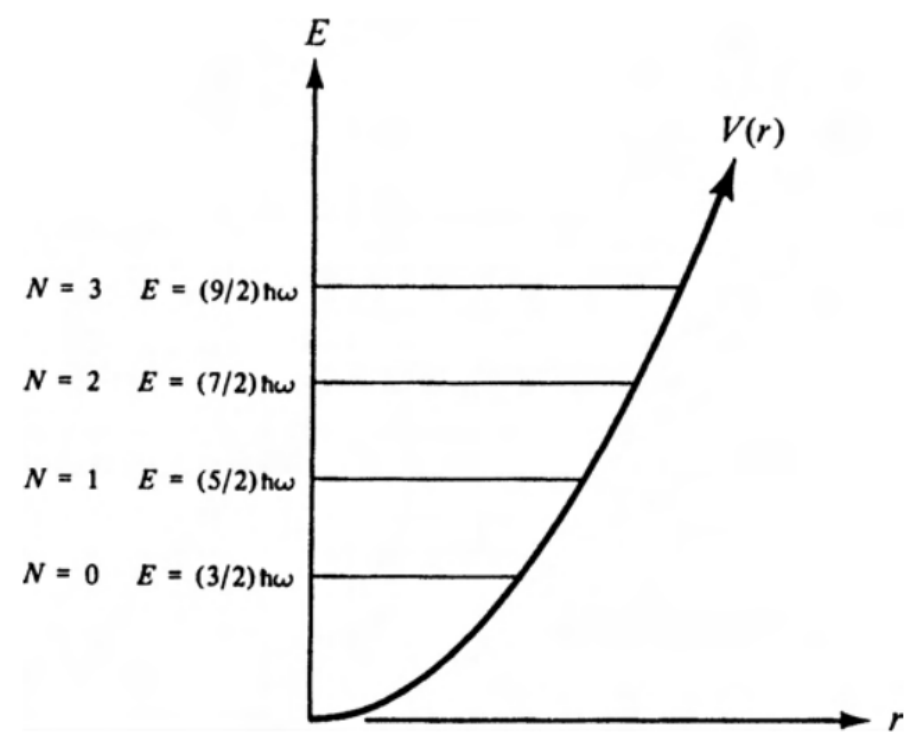

Figure 2.2: Three-dimensional harmonic oscillator and its energy levels [12].

by the interaction between the nucleon spin and its orbital angular momentum. Fig. 2.4 shows the level pattern for nucleons based on "shell model".

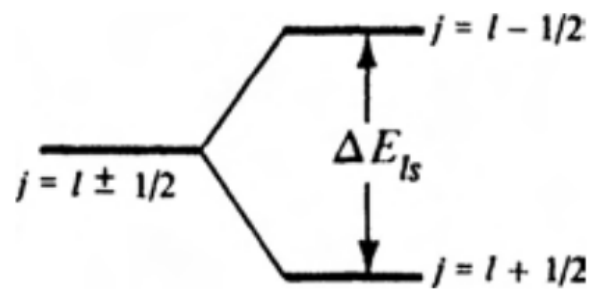

Figure 2.3: Splitting of the states with a given value of $l$ into two states. The spin-orbit interaction depresses the state with total angular momentum $j=l+\frac{1}{2}$ and raises the one with $j=l-\frac{1}{2}$ [12].

\subsection{Collective model}

Although shell model describes the magic numbers and the properties of many levels very well, it has a number of failures. The most outstanding one is that many quadrupole moments are much larger than those predicted by shell model [14]. Such large quadrupole moments can be explained within the concept of a shell model if the closed-shell core is assumed to be deformed [15]. Indeed, if the core is ellipsoidal, it acquires a quadrupole moment proportional to deformation. Quadrupole moment measures the deviation of the shape of the nuclear charge distribution from a sphere. The existence of a quadrupole moment implies non-spherical (deformed) nuclei. The quadrupole moments far away from 


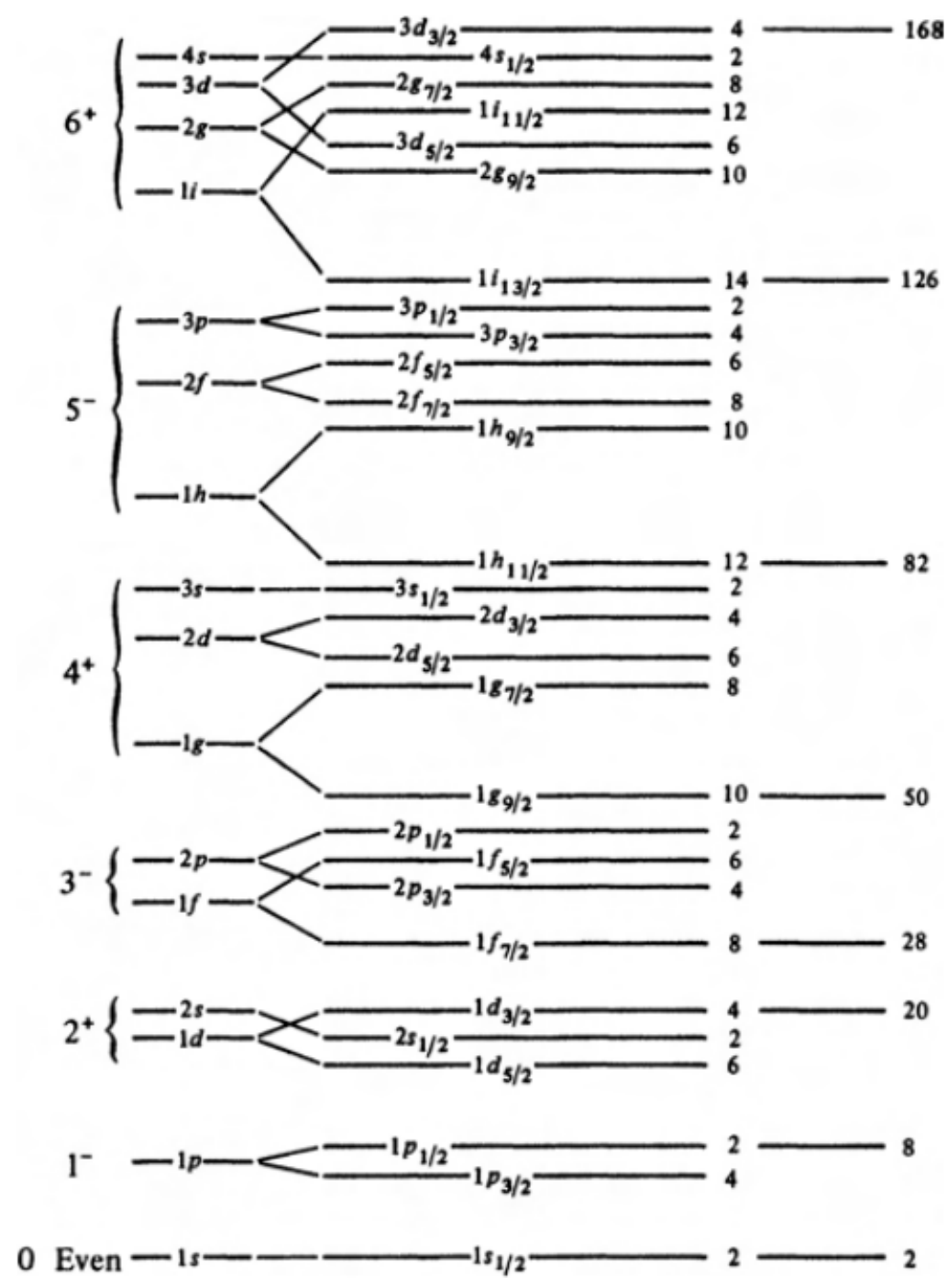

Figure 2.4: Approximate level pattern for nucleons. The number of nucleons in each level and the cumulative totals are shown. Neutrons and protons have essentially the same level pattern up to 50. From then on, some deviations occure [12].

closed shells are so large that they cannot be due to a single particle and thus cannot be explained by the naive shell model [12]. A deformation of core is evidence for many-body effects, and collective modes of excitation are possible. From 1950, Bohr and Mottelson started a systematic study of collective motions in nuclei; over the years, they and their collaborators have improved the treatment so that today the model combines the desirable features of shell and collective models and is called the unified nuclear model [16].

Closed shell nuclei are spherically symmetric and not deformed. The primary collective motions of such nuclei are surface oscillations, like the surface waves on a liquid drop. For small oscillations, harmonic restoring forces are assumed, and equally spaced 
vibrational levels result. Far away from closed shells, the nucleons outside the core polarize the core, and the nucleus can acquire a permanent deformation. The entire deformed nucleus can rotate, and this type of collective excitation leads to the appearance of rotational bands. The deformed nucleus acts as a non-spherical potential for the much more rapid single-particle motion; the energy levels of a single particle in such a potential can be investigated, and the result is the Nilsson model [17].

\subsection{Nilsson model}

The nucleus can be treated by a shell model. Nuclei can also be considered as collective systems that can rotate. Is there a way to weld the two models into one? Yes, the unified model called Nilsson model. Nilsson model considers a deformed nucleus as consisting of independent particles moving in a deformed well.

Since many nuclei possess large permanent deformations, nucleons do not always move in a spherical potential. A well-known generalization is due to Nilsson, who wrote:

$$
V_{d e f}=\frac{1}{2} m\left[\omega_{\perp}^{2}\left(x_{1}^{2}+x_{2}^{2}\right)+\omega_{3}^{2} x_{3}^{2}\right]+C \boldsymbol{l} \cdot \boldsymbol{s}+D l^{2} .
$$

This potential describes an axially symmetric situation - the one that applies to most deformed nuclei. The coordinates $x_{1}, x_{2}$, and $x_{3}$ are fixed in the nucleus: $x_{3}$ lies along the symmetry axis, 3 . $C$ determines the strength of the spin-orbit interaction. The term $D l^{2}$ corrects the radial dependence of the potential: the oscillator potential differs markedly from the realistic potential near the nuclear surface, as shown in Fig. 2.5.

States with large orbital angular momentum are most sensitive to this difference, and the term $D l^{2}$, with $D<0$, lowers the energy of these states. Nuclear matter is nearly incompressible: For a given form of the deformation, the coefficients $\omega_{\perp}$ and $\omega_{3}$ are thus related. For a pure quadrupole deformation, the relation between the coefficients $\omega_{\perp}$ and $\omega_{3}$ is expressed in terms of a deformation parameter $\epsilon$ :

$$
\omega_{3}=\omega_{0}\left(1-\frac{2}{3} \epsilon\right), \quad \omega_{\perp}=\omega_{0}\left(1+\frac{1}{3} \epsilon\right)
$$




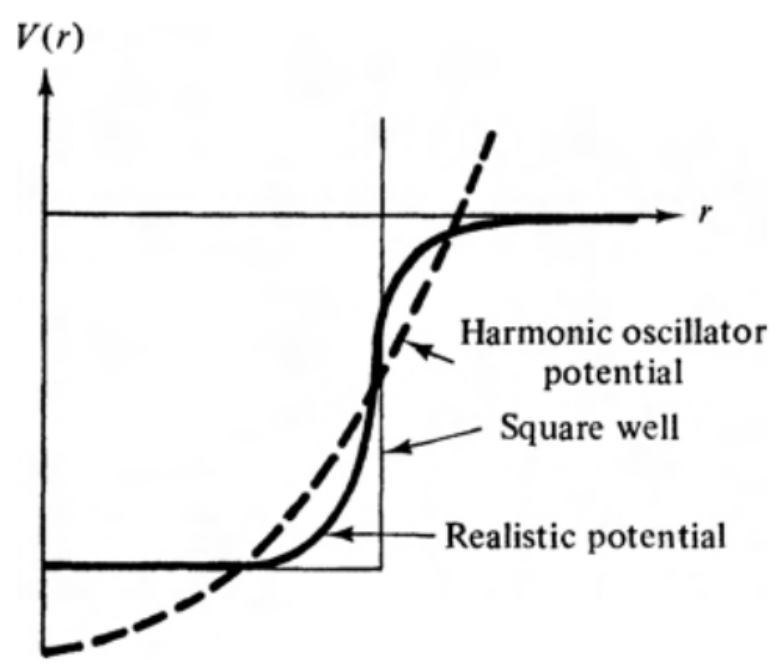

Figure 2.5: The more realistic potential resembling the actual nuclear density distribution is replaced by a harmonic oscillator potential or a square well [12].

For $\epsilon^{2} \ll 1, \omega^{2}$ and $\omega_{3}$ satisfy:

$$
\omega_{\perp}^{2} \omega_{3}=\omega_{0}^{3}
$$

and this relation expresses the constancy of the nuclear volume on deformation. The parameter $\epsilon$ is connected to the deformation parameter $\delta\left(\delta=\frac{\Delta R}{\bar{R}}\right)$ by:

$$
\delta=\epsilon\left(1+\frac{1}{2} \epsilon\right)
$$

The intrinsic quadrupole moment can be written as:

$$
Q=\frac{4}{3} Z\left\langle r^{2}\right\rangle \epsilon\left(1+\frac{1}{2} \epsilon\right)
$$

Eqs. 2.6 and 2.7 show that $V_{d e f}$ is determined by four parameters, $\omega_{0}, C, D, \epsilon$. Only $\epsilon$ depends strongly on the nuclear shape. For a given nuclide, $\epsilon$ is found by measuring $Q$ and $\left\langle r^{2}\right\rangle$. The first three parameters, $\omega_{0}, C$, and $D$, are independent of the nuclear shape for $\epsilon^{2} \leq 1$, and they are determined from the spectra and radii of spherical nuclei, where $\epsilon=0$. Approximate values of these parameters are:

$$
\hbar \omega_{0} \approx 41 A^{-1 / 3}(M e V), \quad C \approx-0.1 \hbar \omega_{0}, \quad D \approx-0.02 \hbar \omega_{0} .
$$


In the Nilsson model, as in the spherical single-particle model, it is assumed that all nucleons except the last odd one are paired and do not contribute to the nuclear moments.

To find the wave function and the energy of the last nucleon, the Schrodinger equation with the potential $V_{d e f}$ is solved numerically with the help of a computer. A typical result for small A is shown in Fig. 2.6. The deformation lifts the degeneracy. State $p_{\frac{3}{2}}$ splits into two and state $d_{\frac{5}{2}}$ into three levels. A nucleon with total angular momentum $j$ in the spherical case gives rise to $\frac{1}{2}(2 j+1)$ different energy levels, with $K$ values $j, j-1, j-2, \ldots, \frac{1}{2}$.

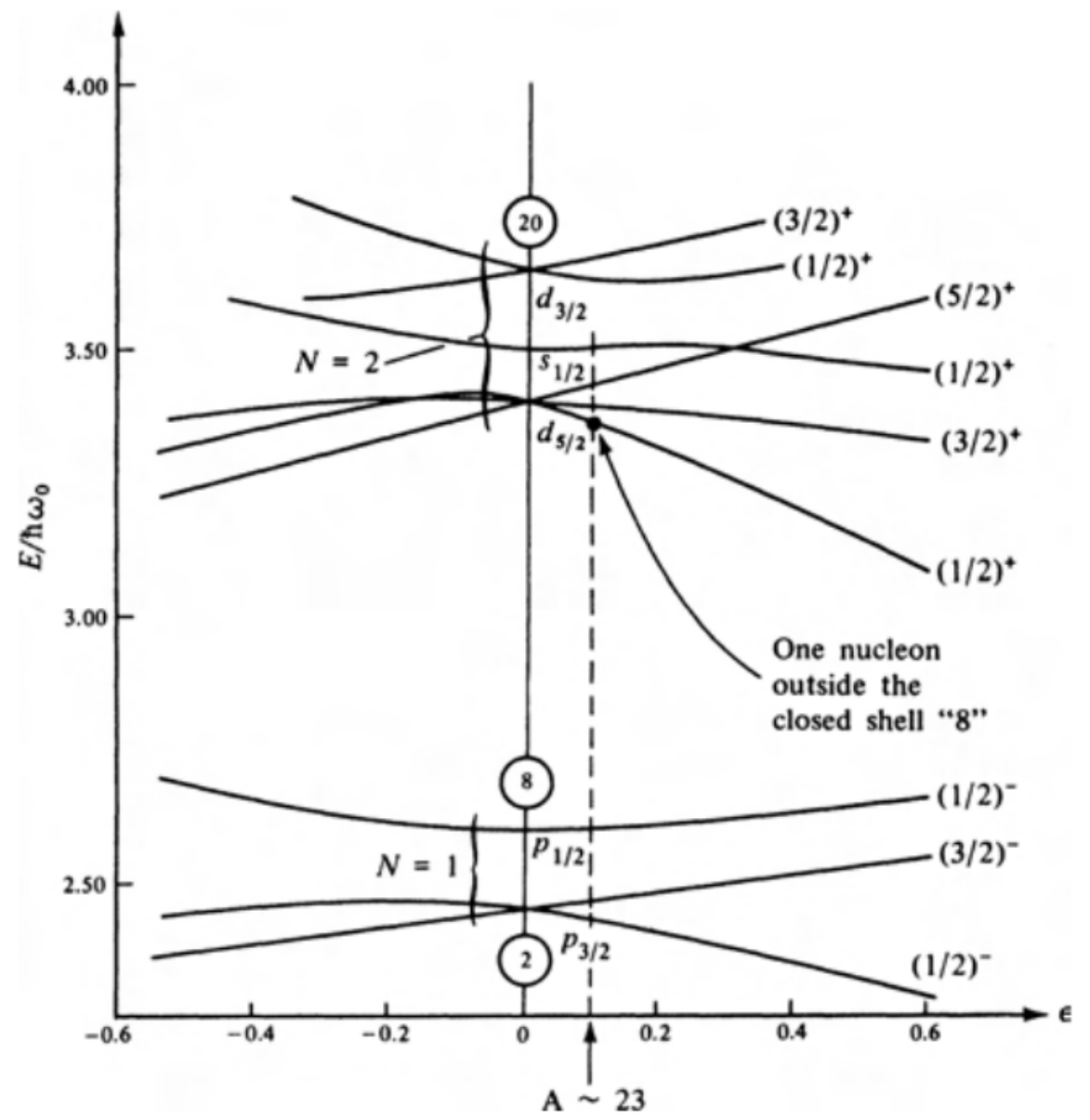

Figure 2.6: Level diagram in the Nilsson model. Each state can accept two nucleons [12]. 


\subsection{Myers-Swiatecki model}

The central idea in Myers-Swiatecki model is that the energy of a nucleus is made up of a liquid-drop part and of a shell correction, the shell correction disappearing for a sufficiently large deformation of the nucleus [18].

From Fig. 2.7, we see that if the shell correction for the sphere is negative, as is the case for a magic nucleus, there results a spherical equilibrium shape with unusual stability. On the other hand, if the shell correction is sufficiently positive (as in a region away from magic numbers), there results automatically a deformed nucleus. The amplitude of the bump goes up and down as one moves across the periodic table in a way indicated in Fig. 2.7. This is the result of assuming the single-particle levels in the spherical potential to be bunched into bands corresponding to the magic numbers. The shape of the bump was assumed to be a Gaussian function of deformation, and is supposed to represent the way in which the bunching disappears as the nucleus is distorted.

Finally, Myers and Swiatecki found the shell damping fiunction is more like the function in Fig. 2.8 than a Gaussian. The difference is that there is one wiggle (or more) following the central bump.

Hence, a function is invented that is like a Gaussian bump in being rapidly damped, but possesses one extra wiggle. The function is the second derivative of a Gaussian:

$$
e^{-\theta^{2}} \text { is replaced by }-\frac{1}{2}\left[e^{-\theta^{2}}\right]^{\prime \prime}=\left(1-2 \theta^{2}\right) e^{-\theta^{2}},
$$

where $\theta$ is a deformation variable, a measure of the eccentricity of the spheroidal nuclear shape.

For a deformed nucleus, the deformation energy now looks like Fig. 2.9.a and the mass sags below the liquid-drop value, which is what we want.

A semi-empirical theory of nuclear masses and deformations is presented. The potential energy of a nucleus, considered as a function of $N, Z$ and the nuclear shape, is assumed to be given by the liquid-drop model, modified by a shell correction. The shell correction is a simple function of $N$ and $Z$ and is supposed to disappear as the nucleus is distorted away from the spherical shape. The resulting semi-empirical expression for the nuclear deformation energy has seven adjustable parameters, four in the liquid-drop part 


\section{Nuclear mass as a function of distortion when the shell correction is:}

(a) negative

(b) positive

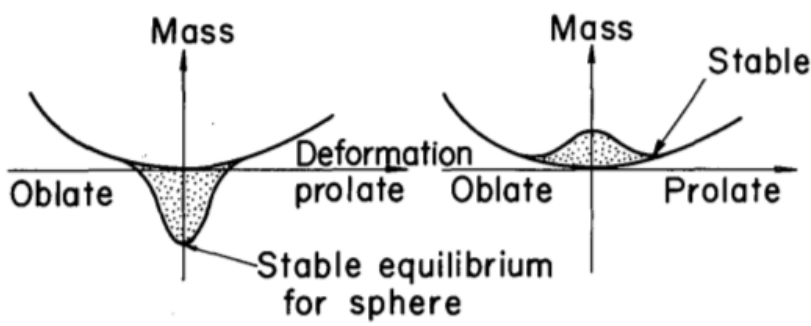

The shell correction $\mathrm{S}(\mathrm{N}, \mathrm{Z})$ along the valley of stability

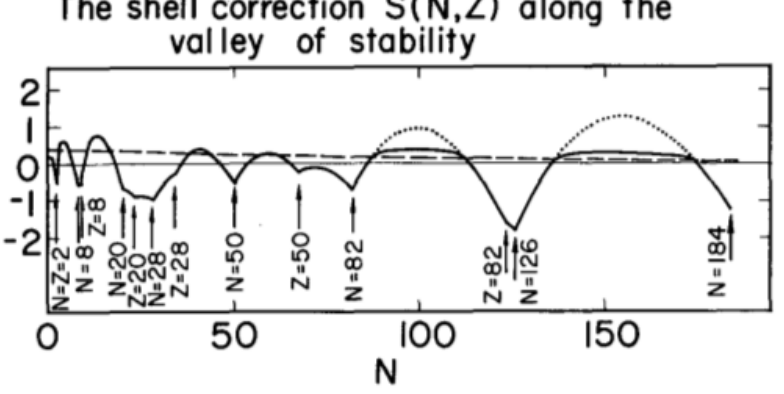

The bunching of levels

in the nuclear well

leads to a shell

correction $\mathrm{S}(\mathrm{N}, \mathrm{Z})$

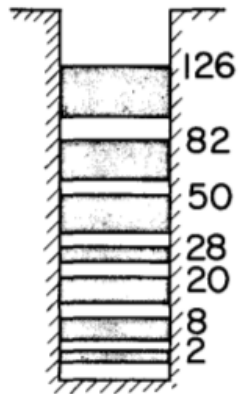

Figure 2.7: The top part of the figure shows schematically how a negative shell correction leads to a spherical shape with special stability, whereas a positive shell correction leads to a deformed equilibrium shape. In the bottom left-hand part, the amplitude of the shell-correction bump along the valley of stability is shown. When the amplitude exceeds a certain critical value, indicated by the dashed line, deformed equilibrium shapes appear. The shell correction is a consequence of the bunching of levels into bands corresponding to magic numbers, as shown schematically on the right [18].

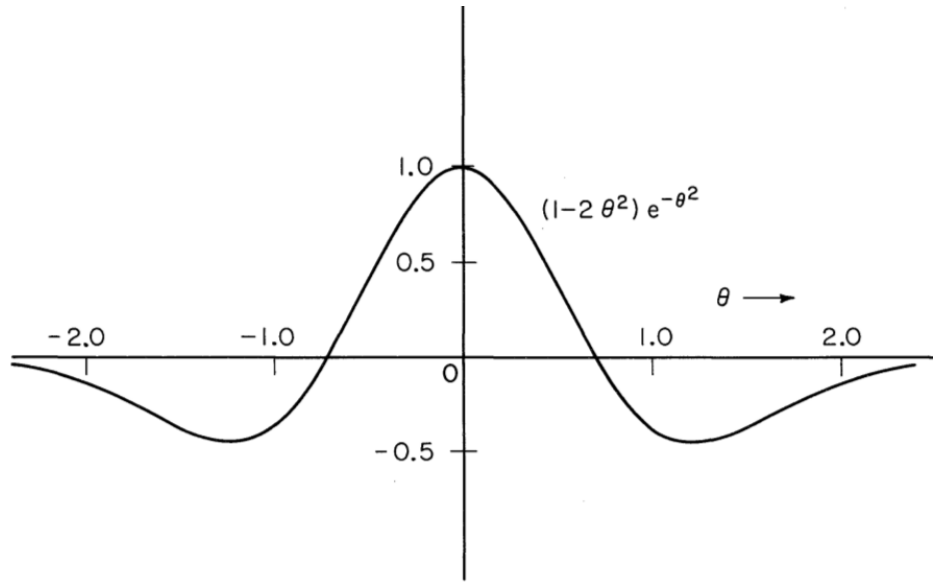

Figure 2.8: This is a plot of the revised shell damping function which replaces the Gaussian function in the original mass formula. Its equation is $\left(1-2 \theta^{2}\right) e^{-\theta^{2}}$, where $\theta$ is a deformation variable [18].

and three in the shell correction. Myers-Swiatecki Revised Mass Formula is [19]:

$$
M=M_{0}+E \theta^{2}-F \theta^{3} \operatorname{Cos}(3 \gamma)+S\left(1-2 \theta^{2}\right) e^{-\theta^{2}},
$$



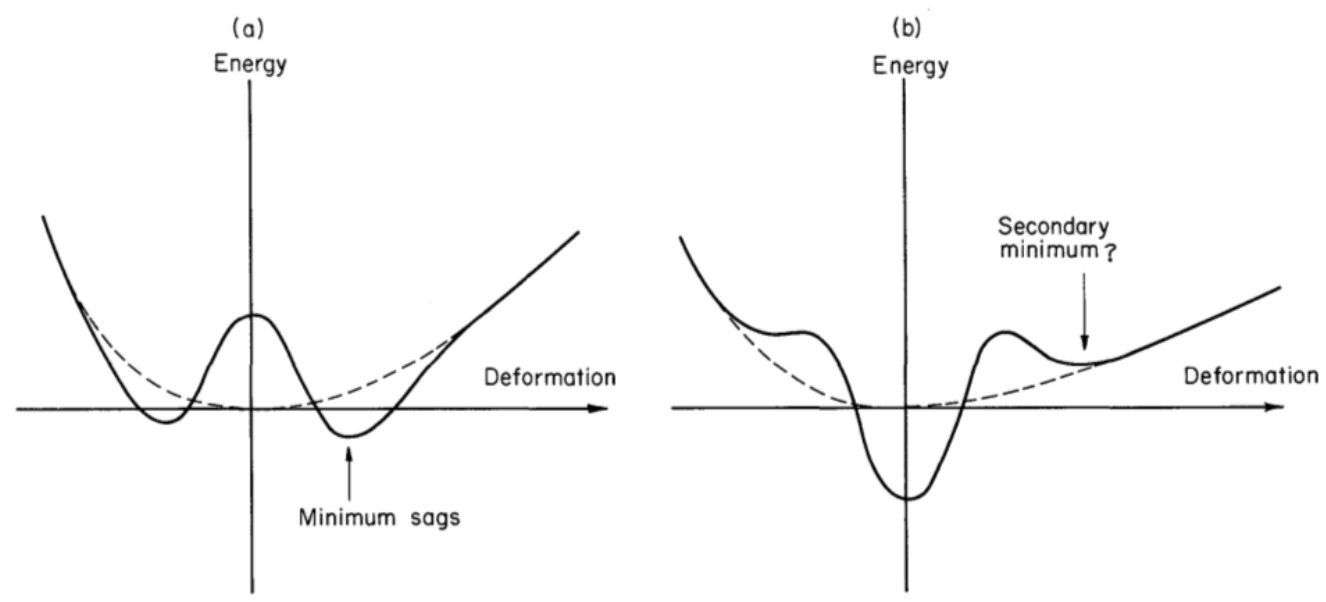

Figure 2.9: The deformation energy is illustrated when the new shell correction is included. In (a) shell correction for the sphere is positive, and this leads to a deformed equilibrium shape whose mass sags below the liquid drop value. In (b) the case of a magic nucleus is illustrated, where a secondary deformed equilibrium shape is predicted [18].

where $\theta$ is the deformation magnitude and:

$$
\begin{aligned}
M_{0} & =M_{n} N+M_{H} Z-c_{1} A+c_{2} A^{2 / 3}+c_{3} \frac{Z^{2}}{A^{1 / 3}}-c_{4} \frac{Z^{2}}{A}+\delta, \\
E & =\left(\frac{2}{5} c_{2} A^{2 / 3}-\frac{1}{5} c_{3} \frac{Z^{2}}{A^{1 / 3}}\right) \alpha_{0}^{2}=\frac{2}{5} c_{2} A^{2 / 3}(1-x) \alpha_{0}^{2}, \\
F & =\frac{4}{105}\left(c_{2} A^{2 / 3}+c_{3} \frac{Z^{2}}{A}\right) \alpha_{0}^{3}=\frac{4}{105} c_{2} A^{2 / 3}(1+2 x) \alpha_{0}^{3}, \\
c_{1} & =a_{1}\left[1-\kappa\left(\frac{N-Z}{A}\right)^{2}\right], \\
c_{2} & =a_{2}\left[1-\kappa\left(\frac{N-Z}{A}\right)^{2}\right], \\
\alpha_{0}^{2} & =5\left(\frac{a}{r_{0}}\right) A^{-\frac{2}{3}}
\end{aligned}
$$

$M_{0}$ is the mass of an undistorted liquid drop, $E$ a coefficient specifying the stiffness of the liquid drop against small spheroidal distortions (and hence related to the fissility parameter $x$, defined as the Coulomb energy term $c_{3} \frac{Z^{2}}{A^{\frac{1}{3}}}$ divided by twice the surface energy $c_{2} A^{\frac{2}{3}}$ ). The coefficient $F$ specifies the cubic term in the liquid-drop formula (through which also the $\gamma$-dependence enters). 
The parameters for Myers-Swiatecki revised mass formula are:

$$
\begin{aligned}
a_{1} & =15.4941 \mathrm{MeV}, & a_{2} & =17.9439 \mathrm{MeV}, \\
c_{3} & =0.7053 \mathrm{MeV}\left(r_{0}=1.2249 \mathrm{fm}\right), & c_{4} & =1.21129 \mathrm{MeV}, \\
\kappa & =1.7826, & C & =5.8 \mathrm{MeV}, \\
c & =0.325, & \frac{a}{r_{0}} & =0.444 .
\end{aligned}
$$

\subsection{Brosa model}

In Brosa model, two models of nuclear exit channel reactions are examined: random neck rupture and multichannel fission. Fission was pictured as a sequence of equilibrium states: the ground or compound state was deemed to be in equilibrium. The same property was attributed to the nucleus at its saddle point. And even at the moment of most violent disintegration, equilibrium was invoked. However, nuclear fission is rather an evolution by instabilities. The word "scission" expresses violent motion somewhat better than "fission". Actually "scission" is intended to denote the instant of rupture [20].

There are several exit channels in spontaneous fission or low-energy induced fission. Leaving the compound state, the nucleus may choose between various paths to disintegration. The term "channel" suggests a guided evolution, and this is exactly what the new channels are for. Instead of one fission barrier - maybe doubly humped - we see that every nuclide has a system of them. Also at rupture, instead of one nuclear shape, several of them can be distinguished. Since there is, in most cases, only one way out of the compound state, fluctuations disregarded, the fission channels must fork. The new objects in multichannel fission are thus bifurcation points.

The main item in random neck rupture is the prescission shape. It looks like two heads connected by a thick neck. Neck rupture means the neck snaps when the nucleus stretches beyond the prescission shape. Random neck rupture means it is not decided where the neck breaks. Knowing random neck rupture, one may compute the most important exit channel observables if the prescission shape is given. However, random neck rupture does not itself deliver the prescission shape. Fortunately, with the fission channel calculations we can find the desired shape (in general several of them). Hence, multichannel calculations and random neck rupture supplement each other. 
Representations of nuclear shapes are suitable for fission if the following conditions are satisfied:

(i) A shape representation must have three essential degrees of freedom: stretching of the nucleus, thinning of the neck and deformation to asymmetry.

(ii) A single sphere and two fragments should be among the allowed configurations.

(iii) The flatness of the neck must be an independent variable.

\subsubsection{Shape parameters}

Suitable set of shape parameters with obvious geometric meanings are (see Fig. 2.10):

$$
l, r, z, c, s
$$

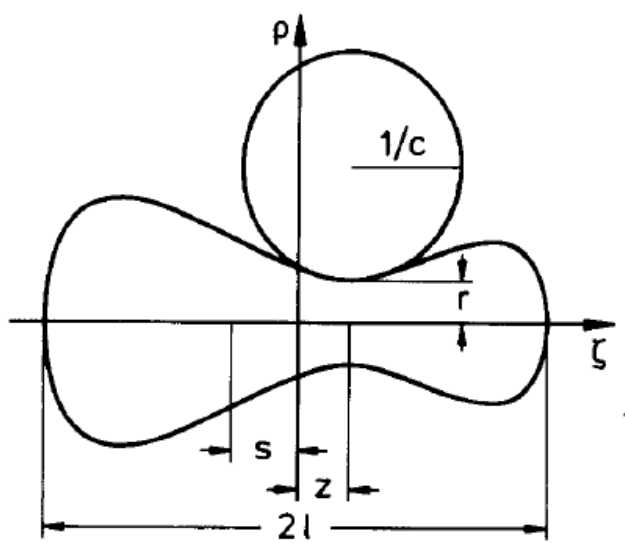

Figure 2.10: Visualization of the degrees of freedom $l, r, z, c$ and $s$. The surface depicted is a special Lawrencian shape displayed in the coordinates $\rho$ and $\zeta$ [20].

The semilength $l$ measures the elongation of the nucleus; we take the semilength instead of the total extension since we want $l$ to coincide with the radius $r_{c n}$ when the shape is a sphere. $r$ is the radius of the neck. As long as there is no neck, $r$ indicates the size of the shape's belly; again, for the spherical "compound nucleus" $r$ agrees with $r_{c n}$. $z$ gives the position on the neck where the neck is thinnest or where the shape is thickest if the neck does not yet exist. $c$ is the curvature of the neck, with positive values if a constriction exists and negative ones in the opposite case. $c$ can be visualized as the inverse of the 
curvature radius. To be precise, we define $c$ as $\frac{r_{c n}^{2}}{r_{c u r}}$. This is not more than the multiplication with a constant and has the advantage of giving $c$ the same dimension as all these parameters, namely length. Finally, $s$ describes the position of the centroid. Hence, $z$ and $s$ are both parameters of asymmetry, and they are both measured relative to the geometric center of the shape.

The parameters $l, r, z, c$ and $s$ have the advantage of being defined for all shapes. The shape function has the form:

$$
\rho=\rho(\zeta) \equiv \rho_{\text {shape }}(\zeta: l, r, z, c, s)
$$

The angle $\phi$ known from cylindrical coordinates $\rho, \phi, \zeta$ does not occur.

\subsubsection{A real flat neck representation (prescission shape)}

A representation which guarantees a "globally flat neck" is:

$$
\rho(\zeta)= \begin{cases}\left(r_{1}^{2}-\zeta^{2}\right)^{\frac{1}{2}} & ;-r_{1} \leq \zeta \leq \zeta_{1} \\ r+a^{2} c\left[\cosh \left(\frac{\zeta-z+l-r_{1}}{a}\right)-1\right] & ; \zeta_{1} \leq \zeta \leq \zeta_{2} \\ \left(r_{2}^{2}-\left[2 l-r_{1}-r_{2}-\zeta\right]^{2}\right)^{\frac{1}{2}} & ; \zeta_{2} \leq \zeta \leq 2 l-r_{1}\end{cases}
$$

This class of shapes is defined for $-r_{1} \leq \zeta \leq 2 l-r_{1}$. An example is depicted in Fig. 2.11. Several of the parameters met in Eq. 2.18 are familiar: the semilength $l$, the neck radius $r$, the position $z$ of the "dent" on the neck, and the neck curvature $c$. A new parameter is the extension $a$ of the neck. With a large $a$, one may keep all higher derivatives small and hence provide for a globally flat neck. The radii $r_{1}$ and $r_{2}$ of the spherical heads are also new, as are the transitional points $\zeta_{1}$ and $\zeta_{2}$ where the three parts of Eq. 2.18 join.

\subsubsection{The embedded spheroids}

When a nucleus scissions, it decays into fragments on which the strong surface tension quickly smooths all the corners and edges. Therefore, we model the newborn fragments as two spheroids in contact (see also Fig. 2.11). 


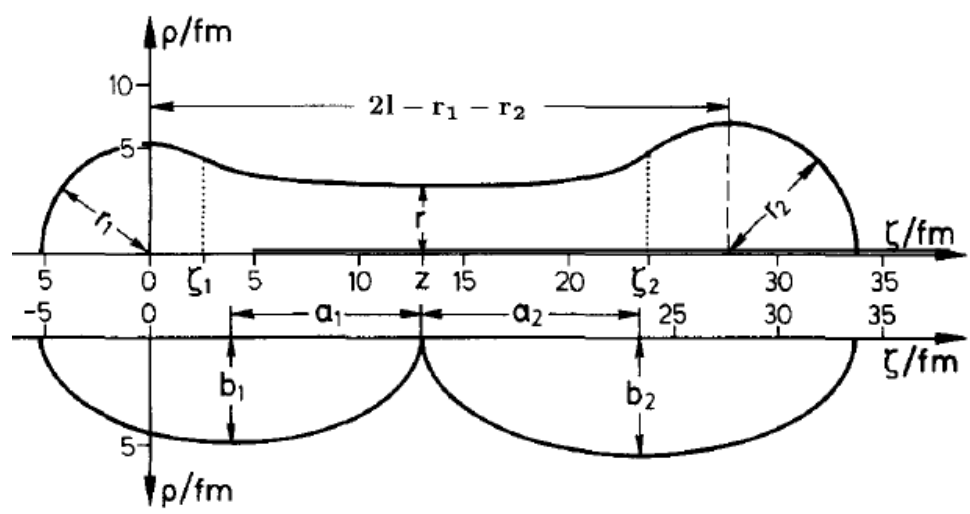

Figure 2.11: The flat-neck representation (Eq. 2.18), upper part; and the embedded spheroids (Eqs. 2.19 and 2.20), lower part. Note the different origin of the coordinates $\rho$ and $\zeta$ compared to that in Fig. 2.10 [20].

Their major axes $a_{1}$ and $a_{2}$ are fixed by the total length $2 l$ and the actual rupture point $z_{r}:$

$$
a_{1}=\frac{1}{2}\left(r_{1}+z_{r}\right), \quad a_{2}=l-\frac{1}{2}\left(r_{1}+z_{r}\right)
$$

The minor axes $b_{1}$ and $b_{2}$ follow from the volume conservation:

$$
b_{1}^{2}=\frac{3}{4 a_{1}} \int_{-r_{1}}^{z_{r}} \rho^{2} d \zeta, \quad b_{2}^{2}=\frac{3}{4 a_{2}} \int_{z_{r}}^{2 l-r_{1}} \rho^{2} d \zeta
$$

These formulas are valid if the coordinate origin is as shown in Fig. 2.11. The embedded spheroids are used to estimate the repulsion between the fragments and the energies of deformation that the fragments have immediately after formation.

\subsubsection{Fundamentals of random neck rupture}

Quantities such as mass yield $Y(A)$, neutron multiplicity $\bar{\nu}(A)$ and total kinetic energy $\overline{T K E}(A)$ are the slaves of the prescission shape. The total kinetic energy $\overline{T K E}$ is an inverse measure of the prescission shape's length. High kinetic energies indicate a short prescission shape, low $\overline{T K E}$ s a long one. The idea behind this is that the nucleus stretches slowly until rupture. The prescission shape is the "last halt". Then the rupture takes place, and Coulomb repulsion accelerates the fragments without any hindrance.

Almost as simple is the relation of the prescission shape to the variance $\sigma_{A}^{2}$ of the mass distribution $Y(A)$. It too measures the prescission shape's length (see Figs. 2.12.a and 2.12.b). More precisely: it measures the length of the neck. Random neck rupture 
produces different fragments by chopping the neck at different positions. The longer the neck, the more possibilities to chop it and the larger the variety of fragments.

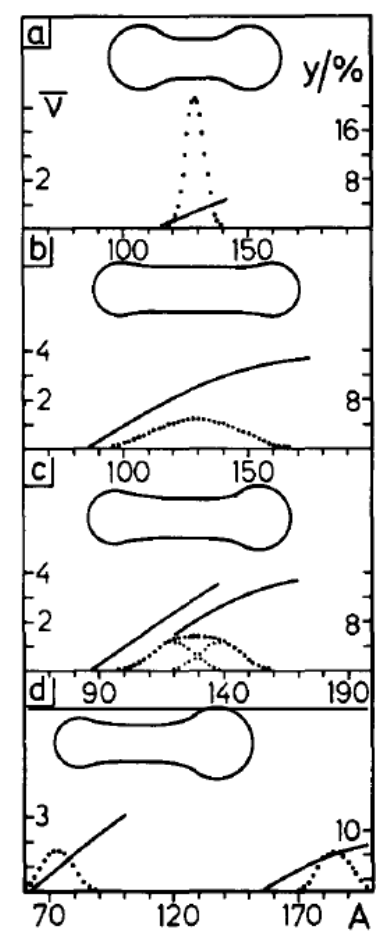

Figure 2.12: Some important correlations that a prescission shape mediates. Shown are mass yields $Y$ (dotted lines, right-hand-side scales) and neutron multiplicities $\bar{\nu}$ (solid lines, left-handside scales) as functions of fragment mass number A. Part (a) shows a supershort prescission shape and its products, part (b) the symmetric, part (c) the standard and part (d) the superasymmetrical prescission shape [20].

The average mass number $A$ of $Y(A)$ expresses the asymmetry of the prescission shape. We expect the most frequent rupture at the place where the neck is thinnest. When the prescission shape is asymmetric, this place is shifted away from the center. Consequently, mostly one light and one heavy fragment are produced, and a double-humped yield $Y(A)$ is obtained, as shown in Fig. 2.12.d.

To understand the neutron multiplicities $\bar{\nu}(A)$, we first state the relations as rules: 1) a large average neutron multiplicity $\bar{\nu}$ is caused by a long prescission shape (see Figs. 2.12.a and 2.12.b); 2) a symmetric prescission shape gives rise to a multiplicity $\bar{\nu}(A)$, which increases steadily with the fragment mass number $A$, (Fig. 2.12.b), whereas an asymmetric prescission shape causes a sawtooth, (Fig. 2.12.d). These rules are based on the embedded spheroids to model the newborn fragments. Their deformations turn into an excitation, and this excitation is finally released by evaporation of neutrons. 
Since long prescission shapes make fragments with large deformations, it is now clear that long prescission shapes give rise to more neutrons, (see rule 1 above). The meaning of rule 2 is detailed in Fig. 2.13: in an asymmetric prescission shape fragments are embedded with about equal masses but very different deformations. They generate the sawtooth.
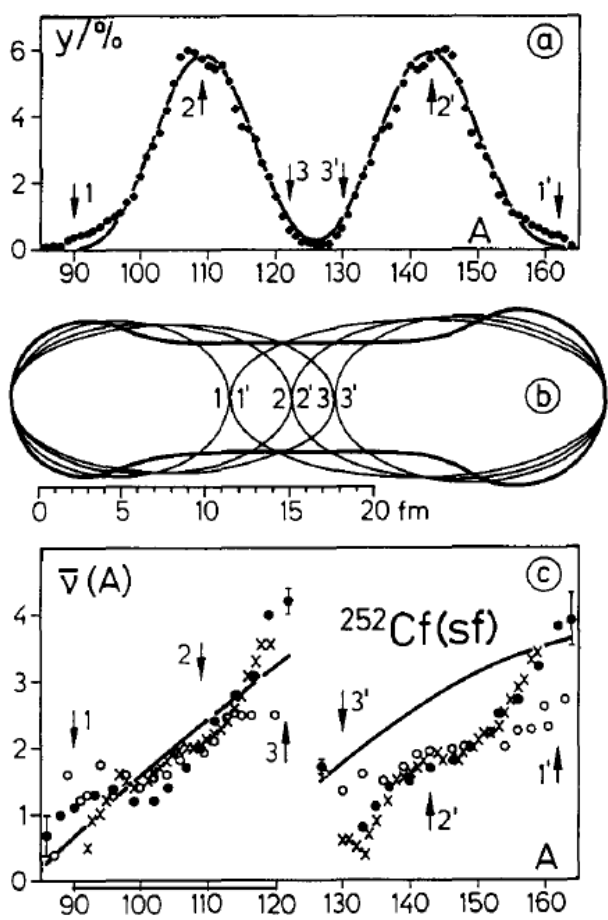

Figure 2.13: Random neck rupture and the sawtooth shape of neutron multiplicity $\bar{\nu}(A)$ [20].

As can be seen in Fig. 2.13: In the central part, (b), the prescission shape valid for the spontaneous fission of ${ }^{252} C f$ is depicted. More precisely, it is approximately the standard prescission shape. Some embedded spheroids are inserted. They are marked by numbers, 2 and $2^{\prime}$, for example. The 2 and $2^{\prime}$ fragments are produced with a large probability because the neck of the prescission shape is thinnest at the $2-2^{\prime}$ position. Therefore, in part (a), arrows 2 and $2^{\prime}$ point at the maxima of the yield $Y(A)$. Rupture at $3-3^{\prime}$, in contrast, rarely happens due to the increased thickness of the neck. It is now most important to notice that the split at $3-3^{\prime}$ gives rise to fragments that are about equal by mass but very different by deformation. As the neutron multiplicity $\bar{\nu}(A)$ increases with deformation, the data shown in part (c) become understandable. Lines represent the results of random neck rupture while experimental material is displayed by symbols. 


\subsubsection{Scission as a sequence of instabilities}

Ordinary fission needs at least three instabilities for its evolution:

(i) passing the barriers

Surmounting the barrier(s) is the element of fission, which was considered from 1939 on. Even today it is sometimes considered as the explanation of fission, though it is only the first step of a complicated walk.

(ii) the shift instability

Shortly behind the last barrier, the neck starts to appear. At first it still has a bump in the middle. Under further stretching the neck becomes perfectly flat, and after this, it will thin in its central part. Shift instability arises, because for fission the nucleus has to change from a spheroidal to a necked-in configuration.

(iii) the capillarity (Rayleigh) instability

Finally, the capillarity or Rayleigh instability ramps. This is the time of constriction. The shift is stopped or, in other words, the asymmetry is frozen, and the nucleus disrupts. Capillarity instability accomplishes what the shift instability prepares. It takes the dent where it is and deepens it until two fragments appear.

\subsubsection{Standard, superlong and supershort}

There are three different prescission shapes: Standard, superlong and supershort. Standard is slightly asymmetric and of "normal" length, while superlong and supershort are both almost symmetrical and appreciably longer or shorter than standard. These differences, being differences in mean length, are usually somewhat larger than those caused by the fluctuations. Hence we expect separable components in the exit-channel observables.

Potential-energy calculations give us the prescission shapes. From prescission shapes, individual yields $Y_{c}(A)$, total kinetic energies $\overline{T K E}_{c}(A)$ and neutron multiplicities $\bar{\nu}_{c}(A)$ are obtained. Subscript $c$ labels the various channels. When we wish to compare with 
measurements, we form the superpositions:

$$
\begin{aligned}
Y(A) & =\sum_{c} p_{c} Y_{c}(A), \quad\left(\mathrm{p}_{c}: \text { channel probability }\right) \\
\overline{T K E}(A) & =\sum_{c} p_{c} \overline{T K E}_{c}(A) Y_{c}(A) / Y(A), \\
\bar{\nu}(A) & =\sum_{c} p_{c} \bar{\nu}_{c}(A) Y_{c}(A) / Y(A) .
\end{aligned}
$$

where channel probabilities $p_{c}$ indicate how much channel $c$ is frequented. It is normalized as $\sum_{c} p_{c}=1$. All functions $Y_{c}(A), \overline{T K E}_{c}(A)$ and $\bar{\nu}_{c}(A)$ are output of procedures of random neck rupture. For information reduction, one may write without loss of accuracy:

$$
\begin{aligned}
Y_{c}(A) & =\frac{1}{\left(2 \pi \sigma_{A, c}^{2}\right)^{1 / 2}}\left[\exp \left(-\frac{\left(A-\bar{A}_{c}\right)^{2}}{2 \sigma_{A, c}^{2}}\right)+\exp \left(-\frac{\left(A-A_{c n}+\bar{A}_{c}\right)^{2}}{2 \sigma_{A, c}^{2}}\right)\right], \\
\overline{T K E}_{c}(A) & =\frac{A\left(A_{c n}-A\right)}{\bar{A}_{c}\left(A_{c n}-\bar{A}_{c}\right)-\sigma_{A, c}^{2}} \overline{T K E}_{c} .
\end{aligned}
$$

so that two functions are defined by four numbers: the average mass $\bar{A}_{c}$, the mass variance $\sigma_{A, c}^{2}$, the average total kinetic energy $\overline{T K E}_{c}$, and the system size $A_{c n}$ given by $\bar{A}_{L}+\bar{A}_{H}$. Random neck rupture delivers these numbers as byproducts.

In Fig. 2.14, channel probabilities $p_{c}$ for the three main fission channels are depicted. The probability of the standard channel is shown by the full lines, the dashed line is related to the superlong channel and the supershort channel is distinguished by the dots.

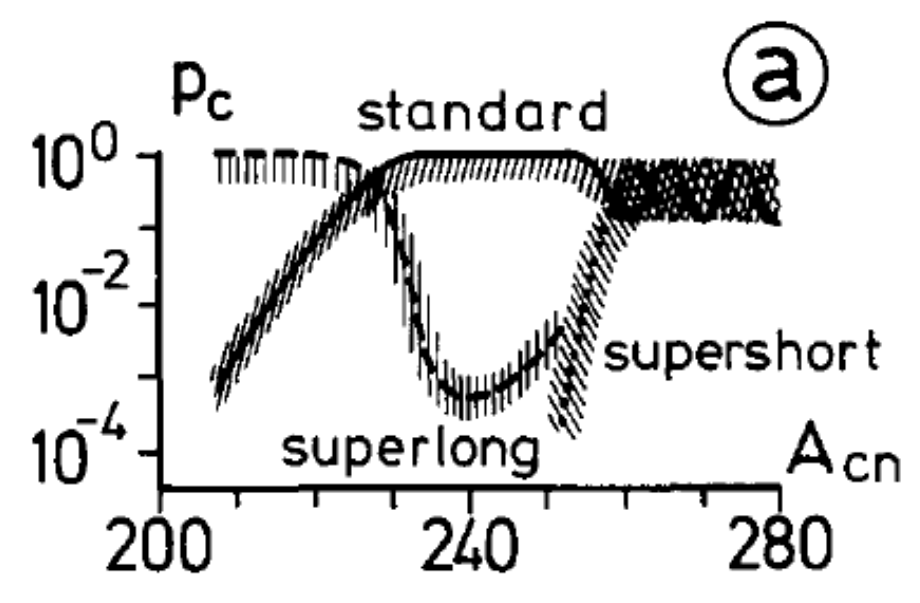

Figure 2.14: Channel probabilities $p_{c}$ as it varies with the size of fissioning system. This is a schematic picture. We have no precise method to compute the channel weights $p_{c}$ [20].

This diagram tells us that for systems smaller than $A_{c n} \approx 250$, the supershort channel 
disappears and the respective probability $p_{s s}$ is zero. For systems larger than $A_{c n} \approx 260$, the superlong channel breaks up. The standard channel, in contrast, exists everywhere. The drastic change of the observables is caused by drastic variation of the population probabilities $p_{c}$. This variation can be related to the relative height of barriers.

For systems smaller than $A_{c n} \approx 250$, where we have a competition between superlong and standard channels, the highest barriers of the channels lie behind the bifurcation, and hence the channels have separate barriers. For systems larger than $A_{c n} \approx 260$, where we have a competition between supershort and standard channels, there is only one highest barrier for both channels. It is located before the bifurcation. Behind the bifurcation, lower secondary barriers may be met. That explains why superlong and standard channels can displace each other, whereas supershort and standard channels must coexist.

\subsubsection{Cayley tree of nuclear fission}

Fig. 2.15 shows the Cayley tree of nuclear fission. Downward motion means an increase of semilength $l$, while motion to the right refers to the growth of asymmetry $z$. We see that the standard channel is slightly asymmetric, while superlong and supershort channels are almost symmetric. There is a splitting of the standard channel into standard I, standard II and perhaps even standard III (ordered with respect to asymmetry). In Fig. 2.15, supershort bifurcation, superlong bifurcation and second standard barrier are shown to be sequential. As can be seen from the figure, the standard II prescission shape is longer than that of the standard I shape. And the biggest shortcoming is the position of the superlong barrier, which has much less elongation than this figure suggests. At the bifurcation points, the nucleus might decide to enter the supershort or superlong channels and to rupture rather at the supershort or the superlong prescission shapes. 


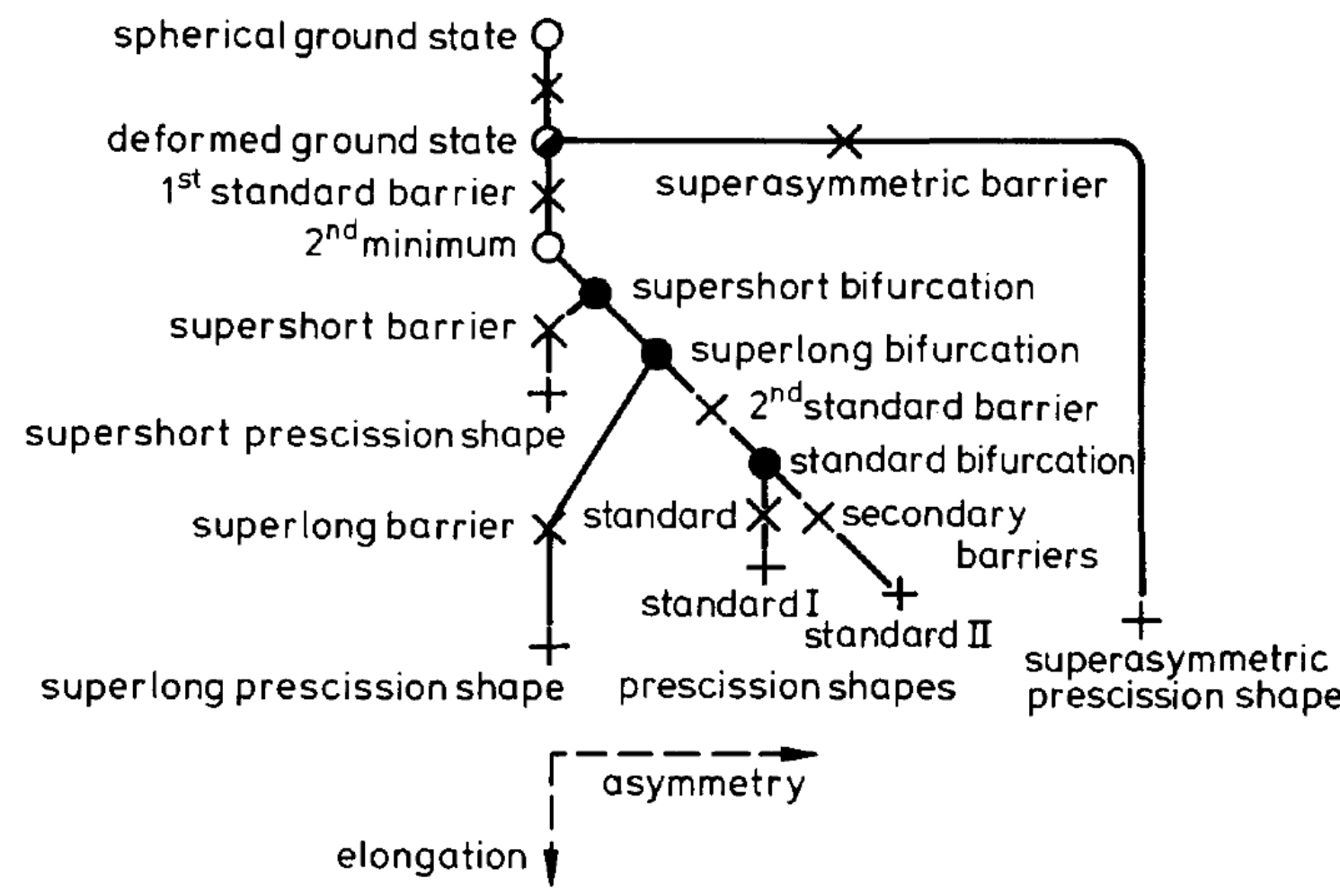

Figure 2.15: Cayley tree of nuclear fission. Emphasized are distinguished points of the potential energy as minima $\circ$, barriers $\times$, bifurcation points $\bullet$, prescission shapes + and their connections (heavy lines) [20]. 


\section{Chapter 3}

\section{CRISP Model}

CRISP model is a simulation code which describes complex characteristics of nuclear reactions using the Monte Carlo techniques [5]. This code has been developed for more than three decades $[5,21,22,23,24,25]$ and has been used to study very different nuclear reactions with success. A lot of good agreements are obtained between experiment and CRISP results for photon-, proton- and electron-induced fission reactions [26, 27, 28, $29,30,31,32,33,34,35,36]$. Also there are some applications of CRISP, especially for nuclear reactions, where we apply the model with success to develop new concepts of nuclear reactors $[37,38,39,40,41]$. Recently, the mechanism of heavy-ion-induced fission has been also added to CRISP model.

At intermediate and high energies, the nuclear reactions can be considered as a twostep process, namely, the Monte Carlo Multi-Collisional (MCMC) intra-nuclear cascade and the Monte Carlo Evaporation-Fission (MCEF) competition mechanism over the excited nuclei resulted from the cascade process.

\subsection{MCMC model}

At the intra-nuclear cascade step, all the processes from the first interaction of projectile with target up to the compound nucleus thermalization are implemented. Since the CRISP model applies the multi-collisional approach in this step, all the nucleons of the target nucleus are involved in the process leading to a more realistic implementation of nuclear reaction mechanisms such as Pauli exclusion principle, nuclear density fluctuations and 
pre-equilibrium emissions [5, 28, 31, 37].

The fast intra-nuclear cascade transfers most of the projectile energy to a few nucleons inside the target nucleus. The implemented time-ordered sequence of collisions considers the probability of interaction with all particles, based on their respective cross sections. Multi-collisional approach makes it possible to verify the full nuclear dynamics such as nuclear density fluctuations and Pauli exclusion principle in a square-well nuclear model $[5,31]$. Without Pauli blocking mechanism, stopping intranuclear cascade is not possible. During the cascade, some particles can escape from the nucleus. When the kinetic energy of each particle inside the nucleus is below its separation energy, the intra-nuclear cascade finishes [28]. At this point, the nucleus thermalization process begins, in which the excitation energy is uniformly distributed among all its nucleons.

\subsection{MCEF model}

In the second step, the evaporation-fission competition is determined by their respective width ratios using the Weisskopf mechanism [42] for evaporation of neutrons, protons and $\alpha$ particles [23, 27, 29] and Bohr-Wheeler model [43] for fission with the fission width calculated according to Vandenbosch and Huizenga [2], and with the help of Pearson nuclear mass formula [44]. The evaporation widths are calculated relative to the neutron width and are recently modified as:

$$
\frac{\Gamma_{p}}{\Gamma_{n}} \simeq\left(\frac{E_{p}}{E_{n}}\right)\left(\frac{a_{n}}{a_{p}}\right) \exp \left\{2\left[\left(a_{p} E_{p}\right)^{\frac{1}{2}}-\left(a_{n} E_{n}\right)^{\frac{1}{2}}\right]\right\}
$$

and

$$
\frac{\Gamma_{\alpha}}{\Gamma_{n}} \simeq\left(\frac{2 E_{\alpha}}{E_{n}}\right)\left(\frac{a_{n}}{a_{\alpha}}\right) \exp \left\{2\left[\left(a_{\alpha} E_{\alpha}\right)^{\frac{1}{2}}-\left(a_{n} E_{n}\right)^{\frac{1}{2}}\right]\right\}
$$

for proton and alpha particle emissions, respectively. And the width ratio for fission process is:

$$
\frac{\Gamma_{f}}{\Gamma_{n}} \simeq K_{f} \exp \left\{2\left[\left(a_{f} E_{f}\right)^{\frac{1}{2}}-\left(a_{n} E_{n}\right)^{\frac{1}{2}}\right]\right\}
$$

where

$$
K_{f}=K_{0} a_{n} \frac{\left[2\left(a_{f} E_{f}\right)^{1 / 2}-1\right]}{\left(4 A^{2 / 3} a_{f} E_{n}\right)}
$$


Table 3.1: Values of the relevant parameters in Dostrovsky's empirical formulas.

\begin{tabular}{cc}
\hline \hline Parameter & Value \\
\hline$a_{1}$ & 18.81 \\
$a_{2}$ & 1.30 \\
$a_{3}$ & 20.07 \\
$a_{4}$ & 3.84 \\
$a_{5}$ & 18.68 \\
$a_{6}$ & 2.02 \\
\hline \hline
\end{tabular}

with $K_{0}=14.39 \mathrm{MeV}$. The level density parameters $a_{n}, a_{p}$ and $a_{\alpha}$ for neutrons, protons and alpha particles are also calculated by Dostrovsky's empirical formulas [45]:

$$
\begin{aligned}
& a_{n}=\frac{A}{a_{1}}\left(1-a_{2} \frac{A-2 Z}{A^{2}}\right)^{2}, \\
& a_{p}=\frac{A}{a_{3}}\left(1-a_{4} \frac{A-2 Z}{A^{2}}\right)^{2}, \\
& a_{\alpha}=\frac{A}{a_{5}}\left(1-\frac{a_{6}}{A}\right)^{2},
\end{aligned}
$$

where the relevant parameters $a_{1}$ to $a_{6}$ for evaporation process are shown in Table 3.1; and $a_{f}=r_{f} \times a_{n}$ where $a_{f}$ is the fission level density parameter with $r_{f}$ being an adjustable parameter.

The energies $E_{i}$ are given by:

$$
\begin{aligned}
& E_{n}=E-B_{n}, \\
& E_{p}=E-B_{p}-V_{p}, \\
& E_{\alpha}=E-B_{\alpha}-V_{\alpha}, \\
& E_{f}=E-B_{f},
\end{aligned}
$$

where $E$ is the excitation energy of the compound nucleus, and $B_{n}, B_{p}$ and $B_{\alpha}$ are the separation energies for neutrons, protons and $\alpha$ particles, respectively, and $B_{f}$ is the fis- 
sion barrier. The Coulomb potentials [46] for proton and alpha particle are, respectively:

$$
V_{p}=C \frac{K_{p}(Z-1) e^{2}}{r_{0}(A-1)^{1 / 3}+R_{p}} \quad \text { and } \quad V_{\alpha}=C \frac{2 K_{\alpha}(Z-2) e^{2}}{r_{0}(A-4)^{1 / 3}+R_{\alpha}}
$$

where the Coulomb barrier penetrabilities for protons and alpha particles are $K_{p}=0.70$ and $K_{\alpha}=0.83$, respectively. Also, the proton radius is $R_{p}=1.14 \mathrm{fm}$ and the alpha particle radius is $R_{\alpha}=2.16 \mathrm{fm}$. $C$ is the charged-particle Coulomb barrier correction due to the nuclear temperature [46]

$$
C=1-\frac{E}{B}
$$

with $B$ being the nuclear binding energy.

After the particle emission at each step $i$ of the evaporation process, the excitation energy of the compound nucleus is recalculated by:

$$
E_{i+1}=E_{i}-B_{i}-V_{i}-\epsilon_{i}
$$

where $\epsilon$ is the kinetic energy of the emitted particles in the evaporation-fission stage. The kinetic energies are calculated by CRISP via Monte Carlo simulation and according to the Weisskopf distribution. The nuclear fission probability, $F_{i}$, is also defined as:

$$
F_{i}=\frac{\left(\frac{\Gamma_{f}}{\Gamma_{n}}\right)_{i}}{1+\left(\frac{\Gamma_{f}}{\Gamma_{n}}\right)_{i}+\left(\frac{\Gamma_{p}}{\Gamma_{n}}\right)_{i}+\left(\frac{\Gamma_{\alpha}}{\Gamma_{n}}\right)_{i}} .
$$

MCEF process continues until the compound nucleus fissions or a heavy residue (spallation product) remains at the end of evaporation phase (since the nucleus excitation energy is not enough to evaporate any other particle). At this point, we can calculate the nuclear fissility [23]:

$$
W=\sum_{i}\left[\prod_{j=0}^{i-1}\left(1-F_{j}\right)\right] F_{i} .
$$

In the evaporation-fission stage, no $Q$-value is calculated because the model does not need it to calculate the probabilities of emission and fission. For fission, it is necessary to know only the total available energy after fission, i.e., final energy after fission $=$ current 
excitation energy - fission barrier.

Both at the evaporation-fission competition stage and at the evaporation of the fission fragments (after the fission happens, the primary fragments may evaporate some nucleons to reach the final fission products), the mass values of nuclides are calculated using the semi-empirical mass formula of Pearson [44]:

$M(A, Z)=Z m_{p}+N m_{n}+a_{v} A+a_{s f} A^{2 / 3}+\frac{3 e^{2}}{5 r_{0}} \frac{Z^{2}}{A^{1 / 3}}+\left(a_{s y m}+a_{s s} A^{-1 / 3}\right) \frac{(N-Z)^{2}}{A}$,

where $e$ is the elementary charge, $a_{v}=-15.65 \mathrm{MeV}, a_{s f}=17.63 \mathrm{MeV}, a_{\text {sym }}=27.72$ $\mathrm{MeV}$ and $a_{s s}=-25.60 \mathrm{MeV}$.

\subsection{Multi-modal fission}

In CRISP, if the nucleus undergoes fission, fission-fragment mass distributions are described by the multimodal random neck rupture (MM-RNR) model [20]. In this model, the prescission shape resembles two heads connected by a thick neck. The nucleus deforms and stretches after the prescission shape. What follows is the rupture of the system at some point along the narrowing. Using MM-RNR model, the fission process continues along several fission channels created by the collective effects of fissioning nucleus deformation in the liquid-drop model and single-particle effects through shell-model corrections which create valleys on the potential energy surface of the fissioning nucleus, each corresponding to one particular fission mode [20, 47, 48].

MM-RNR model combines the picture of multimodal fission with the so-called random neck-rupture phenomenon at scission and also explains the characteristic fission fragment properties successfully. In this model, the fission process is considered to proceed along several deformation channels, and fragment mass distributions are finally calculated by the uncorrelated sum of these individual fission modes, namely, symmetric (S) and asymmetric (AS) modes. Each mode is described by a Gaussian function with the position centered on the most probable mass fragment. The symmetric mode (S) requires only one Gaussian, while the asymmetric (AS) mode is characterized by two Gaussians, one for the heavy fragment and the other for the light one. The positions of the Gaussians, 
dispersion and normalization constants are determined through comparison with the experimental mass distribution. In the symmetric mode, at the saddle-point, the fissioning nucleus (with mass $A_{f}$ ) has an extremely deformed shape with a long neck connecting the two fragments with the most probable masses about $A_{f} / 2$. In the asymmetric mode, the most probable values for heavy and light fragment masses $\left(A_{H}\right.$ and $\left.A_{L}\right)$ are close to one of the shell numbers, due to the single-particle and structure effects in the fission fragments. Fission cross section as a function of mass number $\left(\sigma_{A}\right)$ is obtained by the sum of three Gaussian functions corresponding to the symmetric and asymmetric modes $[49,50,51]:$

$$
\begin{aligned}
\sigma_{A}= & \frac{1}{\sqrt{2 \pi}}\left[\frac{K_{A S}}{\Gamma_{A S}} \exp \left(-\frac{\left(A-\bar{A}_{S}-D_{A S}\right)^{2}}{2 \Gamma_{A S}^{2}}\right)+\frac{K_{A S}^{\prime}}{\Gamma_{A S}^{\prime}} \exp \left(-\frac{\left(A-\bar{A}_{S}+D_{A S}\right)^{2}}{2 \Gamma_{A S}^{\prime 2}}\right)\right. \\
& \left.+\frac{K_{S}}{\Gamma_{S}} \exp \left(-\frac{\left(A-\bar{A}_{S}\right)^{2}}{2 \Gamma_{S}^{2}}\right)\right],
\end{aligned}
$$

where $A$ is the fragment mass number; the positions of the most probable mass for heavy and light fragments are determined as $A_{H}=\bar{A}_{S}+D_{A S}$ and $A_{L}=\bar{A}_{S}-D_{A S}$, respectively, where $\bar{A}_{S}$ is the mean mass number which determines the center of symmetric mode's Gaussian functions; $K_{i}$ and $\Gamma_{i}$ are the intensity and dispersion parameters of each Gaussian function; and the shift $D_{A S}$ determines the center of asymmetric mode's Gaussian functions. The parameter $D_{A S}$ in Eq. (3.13) depends on the nuclear shell structure and on the nuclear excitation energy. In the present work, $D_{A S}$ is used as a free parameter to adjust the calculated distribution to the experimental data.

The Monte Carlo CRISP code follows the history of the entire process from the first interaction of projectile with target up to the fission and spallation, considering all stages in between. Since the fission fragments are unstable nuclei, they are allowed to evaporate by the Weisskopf statistical evaporation model. At the end, the final fission and spallation products shall be compared to the experimental data. Therefore, contrary to experiment, using CRISP model, we can calculate two types of fissioning nuclei: before $\left(A_{f}\right)$ and after $\left(A_{f f}\right)$ post-fission evaporation, where $A_{H}+A_{L}=A_{f f}$ and $\bar{A}_{S}=A_{f f} / 2$.

The fragment charge distribution can also be estimated by considering the Gaussian 
Table 3.2: Parameters used to determine the calculated charge distribution.

\begin{tabular}{cc}
\hline \hline Parameter & Value \\
\hline$\mu_{1}$ & 2.53 \\
$\mu_{2}$ & 0.395 \\
$\gamma_{1}$ & 0.92 \\
$\gamma_{2}$ & 0.003 \\
\hline \hline
\end{tabular}

functions in the form $[52,53]$ :

$$
\sigma(A, Z)=\frac{\sigma(A)}{\Gamma_{Z} \pi^{1 / 2}} \exp \left(-\frac{\left(Z-Z_{p}\right)^{2}}{\Gamma_{Z}^{2}}\right)
$$

where $\sigma(A, Z)$ is the independent cross section of the nuclide $(Z, A), \sigma(A)$ is the total isobaric cross section of the mass chain $A, Z_{p}$ is the most-probable charge for that isobar, and $\Gamma_{Z}$ is the width of the charge distribution. Parameters of charge distribution determine the position of residue nucleus concerning stable isotopes with maximum yield in isobaric chain. The parameters $Z_{p}$ and $\Gamma_{Z}$ are the linear functions of the mass numbers of fission fragments:

$$
Z_{p}=\mu_{1}+\mu_{2} A
$$

and

$$
\Gamma_{Z}=\gamma_{1}+\gamma_{2} A
$$

where the parameter values for $\mu_{1}, \mu_{2}, \gamma_{1}$ and $\gamma_{2}$ are shown in Table 3.2. The CRISP code has adapted to consider the multimodal model by the use of Eqs. (3.13) and (3.14).

Once the fragments $\left(A_{1}, Z_{1}\right)$ and $\left(A_{2}, Z_{2}\right)$ are determined, the excitation energy $E$ is distributed as the excitation energy of the fragments depending on their respective mass numbers. To calculate the kinetic energy of each fragment, we start from the conservation of energy and linear momentum:

$$
T=E+Q=E+M(A, Z)-m_{1}\left(A_{1}, Z_{1}\right)-m_{2}\left(A_{2}, Z_{2}\right),
$$


where $M$ is the mass of the fissioning nucleus, and $m_{1}$ and $m_{2}$ are the masses of fission fragments, all calculated by the Pearson semi-empirical mass formula. The $Q$-value is only implemented here in the calculation of the kinetic energy of fission fragments.

The kinetic energy of fission fragments is calculated by:

$$
T=T_{1}+T_{2}=\frac{p_{1}^{2}}{2 m_{1}}+\frac{p_{2}^{2}}{2 m_{2}},
$$

where $p_{1}$ and $p_{2}$ are the linear momenta of the fragments $\left(p_{1}=p_{2}\right)$. Consequently:

$$
T=T_{1}+T_{2}=\frac{p_{1}^{2}}{2}\left(\frac{1}{m_{1}}+\frac{1}{m_{2}}\right)=\frac{p_{1}^{2}}{2}\left(\frac{m_{1}+m_{2}}{m_{1} m_{2}}\right) .
$$

Therefore, the kinetic energy of each fragment is:

$$
T_{1}=\frac{m_{2}}{m_{1}+m_{2}} T \quad \text { and } \quad T_{2}=\frac{m_{1}}{m_{1}+m_{2}} T .
$$

CRISP model successfully describes nuclear reactions triggered by probes such as photons, protons and electrons [27, 21, 22, 23, 24, 25, 37, 35], and recently, has been extended to consider heavy ions as the incident projectiles. The mechanism of heavy-ion induced nuclear reactions has been covered in a new branch of the Monte Carlo CRISP code in the framework of the rotating liquid drop model.

In the intranuclear cascade stage, we are restricted to the collision of spherical-spherical nuclei. In the evaporation-fission phase, we use the statistical models of Weisskopf and Bohr-Wheeler. They allow the calculation of the probability of emission of protons, neutrons, alpha particles or nuclear fission as a function of $E, A, Z$ and angular momentum $L$ (for nucleus-nucleus collisions). In the equations entered in the latter phase (evaporationfission), CRISP does not consider any specific form of nuclei, it is only necessary to use the values of $E, A, Z$ and $L$. 


\subsection{Additions and improvements of CRISP model}

In this section, I explain the improvement I have done in CRISP model, in the evaporation width ratios formulas.

\subsubsection{Correction in MCEF (evaporation width ratios)}

Based on Weisskopf model, the relative probability of emission of two particles $i$ and $j$ is obtained by:

$$
\frac{\Gamma_{i}}{\Gamma_{j}}=\frac{\gamma_{i}}{\gamma_{j}}\left(\frac{E_{i}}{E_{j}}\right) \frac{a_{j}}{a_{i}} \exp \left\{2\left[\left(a_{i} E_{i}\right)^{\frac{1}{2}}-\left(a_{j} E_{j}\right)^{\frac{1}{2}}\right]\right\}
$$

where $a_{i}$ and $a_{j}$ are the level density parameters; and $\gamma_{i}$ is calculated by:

$$
\gamma_{i}=\frac{g m}{\pi^{2} \hbar^{3}}
$$

where $g$ is the number of states for the spin of particle. $g=2$ for neutrons and protons and $g=1$ for $\alpha$-particles. And $m$ is mass of particle $i . m_{p} \approx m_{n}$ and $m_{\alpha} \approx 4 m_{n}$. Hence:

$$
\begin{aligned}
& \frac{\gamma_{p}}{\gamma_{n}}=\frac{g_{p}}{g_{n}} \times \frac{m_{p}}{m_{n}}=\frac{2}{2} \times \frac{1}{1}=1, \\
& \frac{\gamma_{\alpha}}{\gamma_{n}}=\frac{g_{\alpha}}{g_{n}} \times \frac{m_{\alpha}}{m_{n}}=\frac{1}{2} \times \frac{4}{1}=2 .
\end{aligned}
$$

Consequently, the ratio for proton emission is:

$$
\frac{\Gamma_{p}}{\Gamma_{n}}=\left(\frac{1 E_{p}}{E_{n}}\right)\left(\frac{a_{n}}{a_{p}}\right) \exp \left\{2\left[\left(a_{p} E_{p}\right)^{\frac{1}{2}}-\left(a_{n} E_{n}\right)^{\frac{1}{2}}\right]\right\}
$$

and for $\alpha$-particle emission is:

$$
\frac{\Gamma_{\alpha}}{\Gamma_{n}}=\left(\frac{2 E_{\alpha}}{E_{n}}\right)\left(\frac{a_{n}}{a_{\alpha}}\right) \exp \left\{2\left[\left(a_{\alpha} E_{\alpha}\right)^{\frac{1}{2}}-\left(a_{n} E_{n}\right)^{\frac{1}{2}}\right]\right\} .
$$




\subsubsection{Level density parameters in the old formulas}

The primary level density parameters used in CRISP were based on the following equations [25]. Level density parameter for neutron emission was:

$$
a_{n}=(0.134 A-1.21) \times 10^{-4} A^{2} \quad M e V^{-1} .
$$

And level density parameter for proton and $\alpha$-particle emissions were:

$$
a_{j}=r_{j} a_{n}
$$

where $r_{p}=r_{\alpha}=1$, hence: $a_{p}=a_{\alpha}=a_{n}$. And we see that:

$$
a_{p}=a_{\alpha}=a_{n} \quad \Longrightarrow \quad \frac{a_{n}}{a_{p}}=\frac{a_{n}}{a_{\alpha}}=1
$$

Consequently, the ratios become:

$$
\begin{aligned}
& \frac{\Gamma_{p}}{\Gamma_{n}}=\left(\frac{E_{p}}{E_{n}}\right) \exp \left\{2\left[\left(a_{p} E_{p}\right)^{\frac{1}{2}}-\left(a_{n} E_{n}\right)^{\frac{1}{2}}\right]\right\}, \\
& \frac{\Gamma_{\alpha}}{\Gamma_{n}}=\left(\frac{2 E_{\alpha}}{E_{n}}\right) \exp \left\{2\left[\left(a_{\alpha} E_{\alpha}\right)^{\frac{1}{2}}-\left(a_{n} E_{n}\right)^{\frac{1}{2}}\right]\right\} .
\end{aligned}
$$

\subsubsection{Level density parameters in the new formulas}

In the last few years, Dostrovsky's empirical formulas [45] are applied in CRISP model to calculate level density parameters:

$$
\begin{aligned}
& a_{n}=\frac{A}{a_{1}}\left(1-a_{2} \frac{A-2 Z}{A^{2}}\right)^{2}, \\
& a_{p}=\frac{A}{a_{3}}\left(1+a_{4} \frac{A-2 Z}{A^{2}}\right)^{2}, \\
& a_{\alpha}=\frac{A}{a_{5}}\left(1-\frac{a_{6}}{A}\right)^{2},
\end{aligned}
$$

which means:

$$
a_{n} \neq a_{p} \neq a_{\alpha}
$$

Therefore, $\frac{a_{n}}{a_{p}}$ and $\frac{a_{n}}{a_{\alpha}}$ should not be omitted! 
Hence, the evaporation width ratios are modified as the following:

The ratio for proton emission becomes:

$$
\frac{\Gamma_{p}}{\Gamma_{n}}=\left(\frac{E_{p}}{E_{n}}\right)\left(\frac{a_{n}}{a_{p}}\right) \exp \left\{2\left[\left(a_{p} E_{p}\right)^{\frac{1}{2}}-\left(a_{n} E_{n}\right)^{\frac{1}{2}}\right]\right\}
$$

and for $\alpha$-particle emission becomes:

$$
\frac{\Gamma_{\alpha}}{\Gamma_{n}}=\left(\frac{2 E_{\alpha}}{E_{n}}\right)\left(\frac{a_{n}}{a_{\alpha}}\right) \exp \left\{2\left[\left(a_{\alpha} E_{\alpha}\right)^{\frac{1}{2}}-\left(a_{n} E_{n}\right)^{\frac{1}{2}}\right]\right\}
$$

To compare between the old formulas used in CRISP (Eqs. (3.30) and (3.31)) and the corrected formulas (Eqs. (3.34) and (3.35)), we considered the projectile proton with initial energy of $1000 \mathrm{MeV}$ colliding with the target nucleus ${ }^{238} \mathrm{U}$, and compared the results of two formulas for the spallation for a fixed atomic number $Z=88$ (Fig. 3.1). We also compared the results of fission for a fixed atomic number $Z=90$ (Fig. 3.2).

As expected, due to the small differences in the values of level density parameters, their ratios $\left(\frac{a_{n}}{a_{p}}\right.$ and $\left.\frac{a_{n}}{a_{\alpha}}\right)$ are near 1 , and hence, the difference between the old formulas and the corrected formulas is not significant. Although the correct formulas have been now inserted in CRISP. 
- old formula
— new formula

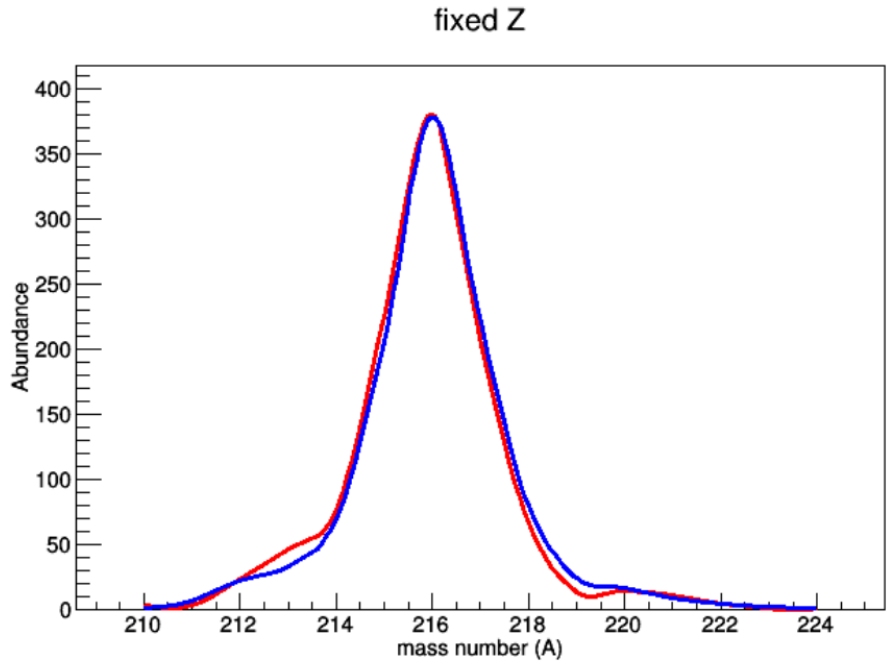

Figure 3.1: Comparing the results of the spallation for a fixed atomic number $Z=88$ using the old formulas in CRISP and the corrected formulas.

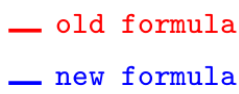

fixed $Z$

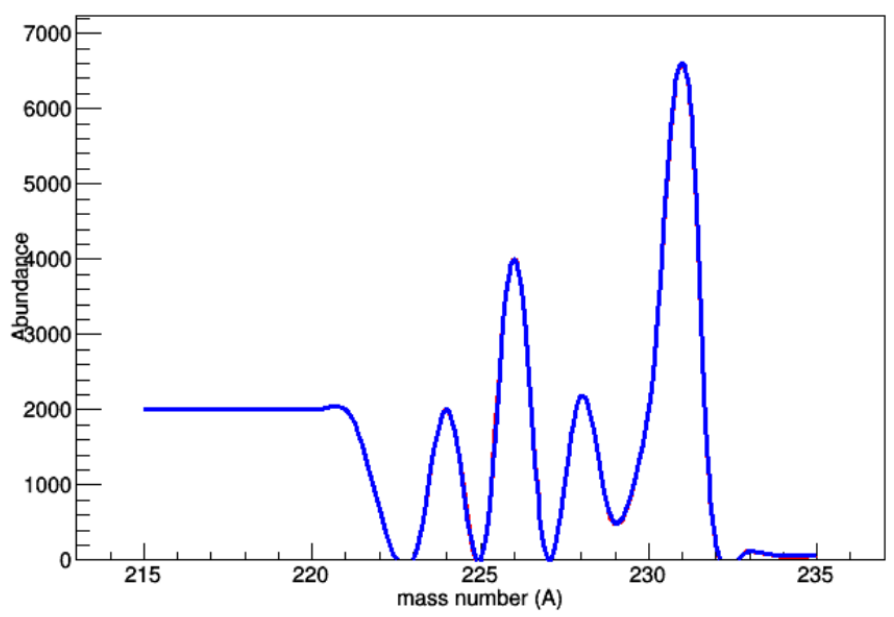

Figure 3.2: Comparing the results of the fission for a fixed atomic number $Z=90$ using the old formulas in CRISP and the corrected formulas. Both results are identical. 


\subsubsection{Implementation of heavy-ion reactions}

\subsubsection{Ion Event Generator}

A new research line is started in CRISP model to investigate the heavy-ion collisions. For this aim, a new event generator is created by one the collaborators to provide the possibility to run intra-nuclear cascade models for ion incident beams. The advantage of the implemented model is that it works much better for light ions, compared to past that we considered the incident ion as a bunch of particles that could be added to the nuclear structure of the target.

\subsubsection{Angular-momentum-dependent fission barrier}

In the present study, using the ion event generator added in the cascade stage, I improved the mcef stage by introducing the rotating finite range model (RFRM) $[54,55]$ (which is a model to calculate the angular-momentum-dependent fission barrier, especially for heavyion collisions) and adding the angular-momentum-dependent fission barrier to CRISP by implementing RFRM model in C++ language and making it compatible with CRISP code and finally, extending it with Nix model. The details of this improvement and the results of the CRISP model are expressed in the following chapters. 


\section{Chapter 4}

\section{Heavy-ion-induced nuclear reactions}

\subsection{Nucleus-nucleus collision model in CRISP}

In heavy-ion-induced nuclear reactions, we have a projectile nucleus $\left(a_{1}, z_{1}\right)$ with energy $T$ that is launched against a target nucleus $(A, Z)$. The trajectory of the projectile nucleus is influenced by the Coulomb potential between the colliding nuclei, given by:

$$
V_{c}=\frac{z_{1} Z e^{2}}{r}
$$

where $r$ is the distance between the centers of charge. The trajectory of the projectile nucleus is classically determined by this potential until the moment of contact. At this moment, the process of nucleonic transference starts in the direction projectile-target [56].

\subsubsection{Initial conditions and point of contact}

The first part of the problem consists of determining the coordinates of the nuclei when contact is made. This can be done in the relative coordinate system of the nuclei, which is represented in Fig. 4.1, where $\vec{r}$ is the relative position vector and $\phi$ is angle between $\vec{r}$ and the positive $z$-direction which is the direction in which the projectile moves.

In this system, the trajectory is described by [57]

$$
\frac{A}{r}=\epsilon \cos \left(\phi-\phi_{0}\right)-1
$$




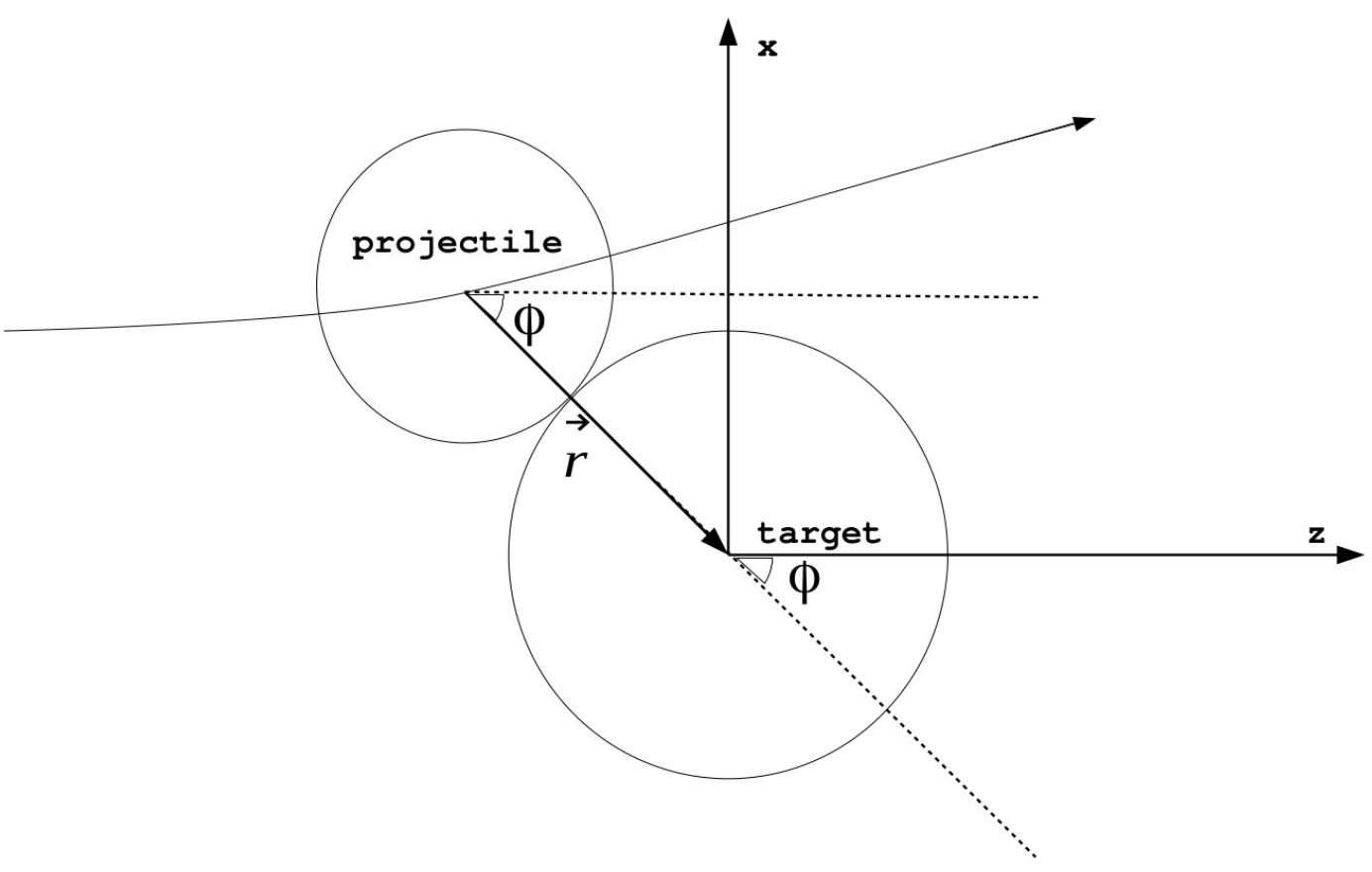

Figure 4.1: System of coordinates used to describe the nucleus-nucleus collisions in CRISP [56].

with parameters given by

$$
\begin{aligned}
A & =\frac{l^{2}}{r \alpha}, \\
\epsilon & =\sqrt{1+\frac{2 T l^{2}}{\mu \alpha}}, \\
\phi_{0} & =\cos ^{-1}\left(\frac{1}{\epsilon}\right) .
\end{aligned}
$$

Here, $l$ is the angular momentum, $\alpha=z_{1} Z e^{2}, T$ is the kinetic energy of the two-body system in relation to its relative velocity. As we are only interested in the classical solution of the Rutherford dispersion, we consider the reduced mass of the system $\mu=\frac{A_{1} A_{2}}{A_{1}+A_{2}}$. In the intranuclear cascade, we are considering transferring nucleons, one by one, from the projectile nucleus to the target nucleus. Since the energy of all nucleons and the potentials of the nuclei are known, it is possible to balance the energy without explicitly using the $Q$-value.

The angular mometum is randomly chosen between 0 and the maximum value $l_{\max }$ in 
such a way that [58]

$$
l=l_{\max } \sqrt{s}
$$

where $s$ is a random number uniformly distributed in the interval $[0,1]$. The maximum angular momentum, $l_{\max }$, refers to the maximum impact parameter $b=R_{1}+R_{2}+d r$ such that

$$
l_{\text {max }}=b P
$$

where $P$ is the momentum of the two-body system in the relative reference frame and $R_{1}$ and $R_{2}$ are the radii of the nuclei. $d r=2 \mathrm{fm}$ is considered for the peripheral collisions, explained in detail in Sec. 4.1.3.

In the relative reference frame, there are four generalized variables, $r, p_{r}, \phi$ and $l$. They obey the following initial conditions:

$$
\begin{aligned}
& r_{0}=\infty, \\
& p_{0}=\sqrt{2 \mu T}, \\
& \phi_{0}=0, \\
& l_{0}=l .
\end{aligned}
$$

In this way, the nuclei make contact if Eq. (4.2) has a solution for the angular coordinate when $r=R_{c}=R_{1}+R_{2}+d r$. In this point, the generalized coordinates assume the values

$$
\begin{aligned}
r & =R_{c}, \\
p & =\sqrt{2 \mu\left(T-V_{c}\left(R_{c}\right)\right)}, \\
\phi & =\phi_{0}-\cos ^{-1}\left(\frac{\frac{A}{R_{c}}+1}{\epsilon}\right), \\
l & =l .
\end{aligned}
$$


The cartesian coordinates are expressed as follows

$$
\begin{aligned}
& \vec{r}=(-r \sin \phi, 0, r \cos \phi), \\
& \vec{p}=(p \sin (\theta-\phi), 0, p \cos (\theta-\phi)), \\
& \theta=\sin \left(\frac{l}{r p}\right) .
\end{aligned}
$$

In order to take into account the three dimensions of the cartesian space, a random rotation around the $z$-direction is performed.

\subsubsection{Nucleonic transference from projectile to target}

At the moment of contact between the nuclei, the possibility that the nucleons from the projectile reach the target surface and initiate an intranuclear cascade must be considered. For each nucleon of the projectile that reaches the target, the interaction is similar to the nucleon-induced reactions already calculated by CRISP.

At the moment of contact, two events are taken into account regarding the nucleons from the projectile: their interaction with the surface of the projectile and with the surface of the target. If a nucleon of the projectile interacts with the projectile's surface, it is reflected in such a way as to conserve the tangencial component of the linear momentum and invert the radial component. On the other hand, if the nucleon hits the target, the transference is done in a way that guarantees energy conservation and follows the Pauli exclusion principle. In addition, a Lorentz transformation is performed since, by default, the movements of the nucleons are described according to a reference frame located always at the center of their own nucleus. In other words, at the moment of nucleonic transference, the entire description is moved to the reference frame at the center of the target. The intranuclear cascade inside the target nucleus then follows.

No disipative process is considered in the initial phase of the reaction. Indirectly, when a nucleon is transferred, the Lorentz equations have to be applied to calculate the momentum in the target nucleus referential, thus the momentum of that nucleon (after being transferred) depends on its initial momentum in the Fermi gas of the projectile nucleus and of the collective momentums of the nuclei. Therefore, when that nucleus 
transfers momentum in future collisions, it transfers energy between the collective and intrinsic degrees of freedom as well.

\subsubsection{Peripheral collisions}

During the calculation of the interaction between two nuclei, one could choose to select the interaction of only those nucleons from the projectile that make geometric contact with the target's surface, considerting a radius $R=r_{0} A^{1 / 3}$ for the target. However, this strict selection leaves out the interaction of nucleons that pass very close to the target, a situation not very realistic. After careful study of the nucleon-nucleon interaction cross sections already implemented in CRISP, it was observed that above $100 \mathrm{MeV}$ the interaction can occur at an impact parameter no larger than $2 \mathrm{fm}$. This fact was taken into account by considering an effective radius for the target given by $R+d r$, with $d r=2 \mathrm{fm}$. This consideration is made only in the context of the initial interaction.

\subsection{Excitation energy and angular momentum}

Let's suppose the following reaction:

$$
\left(a_{1}, z_{1}\right)+(A, Z)=\left(A_{f}, Z_{f}\right)+\text { nucleons. }
$$

Let's assume that $A_{f}<A$. In order to calculate the excitation energy, a new nucleus $(A, Z)$ is constructed at ground state and $A-A_{f}+Z-Z_{f}$ neutrons and $Z-Z_{f}$ protons are removed. The result is necessarily a $A_{f}, Z_{f}$ nucleus at ground state. The excitation energy is the difference between the kinetic energy of all nucleons in the actual residual nucleus and the ground state version. This calculation is expressed by

$$
E^{*}=\sum_{i=1}^{A_{f}} T_{i}-\sum_{i=1}^{A_{f}} T_{i}^{\prime},
$$

where $T_{i}$ is the kinetic energy of the $i$ th nucleon of the residual nucleus and $T_{i}^{\prime}$ is the kinetic energy of the $i$ th nucleon of the ground state version of the same nucleus.

In case $A_{f}>A$, the excitation energy is the difference between the kinetic energy of all nucleons that entered the target and the ones that left. 
The angular momentum is calculated as:

$$
L=\sum l_{\text {in }}-\sum l_{\text {out }}
$$

where $l_{\text {in }}$ is the angular momentum of the entering nucleon, coming from the projectile nucleus, and $l_{\text {out }}$ is the angular momentum of the emitted nucleon.

\subsection{Angular momentum in heavy-ion-induced nuclear re- actions}

The angular momentum imparted by the heavy-ion projectile to the target nucleus at intermediate energies is considerable compared to the nucleon-nucleus and photonuclear interactions. Based on the dynamic model described in [59], in heavy-ion induced fusionfission processes, the initial relative kinetic energy and angular momentum of the projectile are converted into the intrinsic excitation energy and spin of the fused compound system leading to the increase of fission probability [60]. The concept of the dynamical force equilibrium is considered to determine the critical, maximum and average angular momenta in the entrance reaction channel. This equilibrium is determined by the balance of the macroscopic surface energy, Coulomb energy, and the rotational energy. The critical angular momentum for the compound system is the critical value up to which projectile and target can still fuse. For angular momenta higher than this critical value, the contact configuration of projectile and target nuclei cannot fuse and will separate so the compound nucleus is not formed.

Since the angular momentum transferred in heavy-ion reactions is significant, the rotating liquid drop model and the effects of angular momentum on lowering fission barriers have been studied since 1960s [61, 62, 63, 64, 65], although it took one decade so that heavy-ion research gains its popularity among the nuclear physicists. At that time, the fission barrier dependence on angular momenta of rotating nuclei was discussed by PikPichak [63] showing that by the increase of angular momentum, fission-barrier height decreases. Fig. 4.2 depicts a general behaviour of this dependency of fission barriers on angular momenta in heavy-ion induced nuclear reactions. Several models present this 
fission barrier dependence on angular momenta of rotating nuclei $[59,60,66,54,55]$.

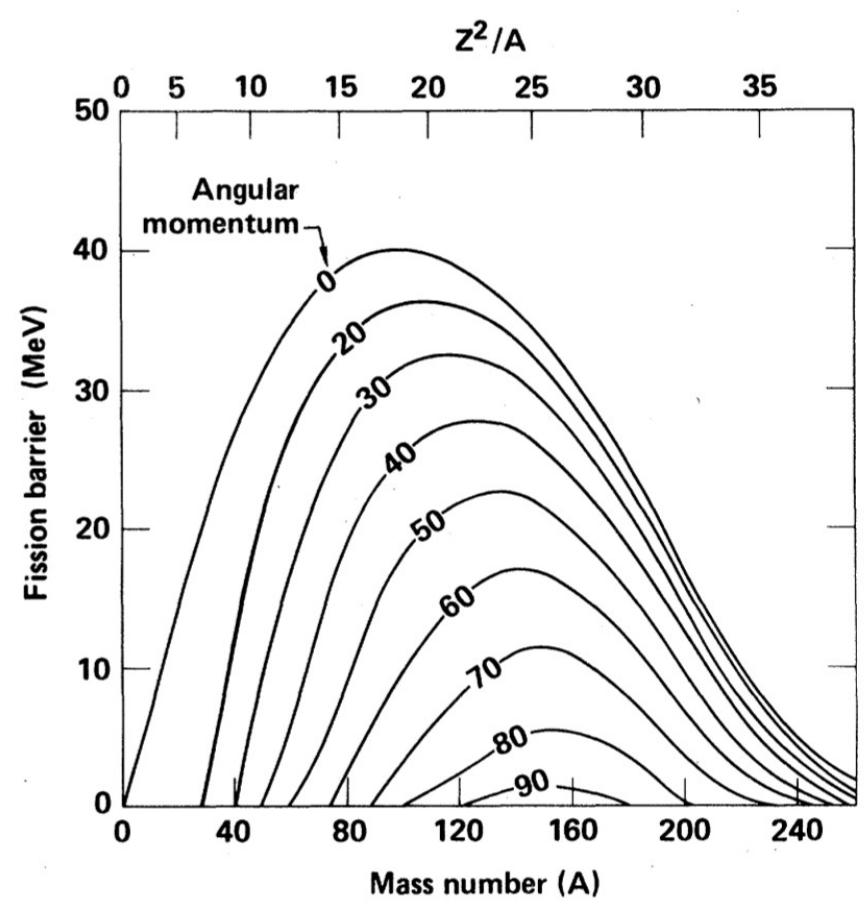

Figure 4.2: General predictions of fission barriers for different angular momenta [54].

\subsubsection{Rotating liquid drop model (RLDM)}

One of these models is the standard rotating liquid drop model (RLDM) introduced by Cohen, Plasil and Swiatecki [66]. In this model, it is assumed that a rotating nucleus can be described by a uniformly rotating, macroscopic liquid drop. This model predicts that, for nucleus-nucleus interactions, the angular momentum transferred by the accelerated heavy ion during the reaction leads to the decrease of fission barrier and consequently increase of fission cross section. However, this dependence can be neglected in the case of nucleon-nucleus and gamma-nucleus interactions at intermediate energies, since only a small angular momentum is imparted to the nucleus by incident nucleons.

The angular-momentum-dependent fission barrier height, $B_{f}(A, Z, L)$, can be calculated by the rotating liquid drop model. However, the analyses performed by this model resulted in higher fission barriers compared to the experimental data showing that for heavy-ion induced fission and spallation, the rotating liquid drop model written by Cohen et al. [66] cannot reproduce the experimental data, and the calculated fission-barrier heights had to be modified by an appropriate scaling factor which has been reported to 
be between about 0.5 and $0.9[67,68,69,70,71,72,73,74]$. This reduced factor value changes rather arbitrarily for different reactions and no systematic trend has been derived among them [75].

\subsubsection{Rotating finite range model (RFRM)}

In 1980s, the rotating finite range model (RFRM) presented by Mustafa et al. [54] and Sierk [55] was revealed as a refinement in RLDM. The improvements in RFRM in comparison with RLDM are in the nuclear shapes and in the calculations of the surface, Coulomb, and rotational energies. In RFRM model, equilibrium shapes and fission barriers of rotating nuclei have been calculated using a macroscopic two-center model, with a finite-range nuclear force and a diffuse nuclear surface $[54,55]$. The two-center-model shape parametrization allows for triaxial shape variations and a continuous transition from one-center to two-center shapes with a smooth neck. The surface energy is calculated with the Yukawa-plus-exponential folding function of Krappe, Nix, and Sierk [76], which incorporates the effects of the finite range of the nuclear force and the diffuse nuclear surface, and both the Coulomb and rotational energies are calculated with surface diffuseness described by a Yukawa folding function.

To itemize the changes of the Sierk macroscopic model of rotating nuclei (RFRM) relative to the RLDM:

- The surface energy of the liquid-drop model is replaced by the Yukawa-plus-exponential nuclear energy, which models effects of the finite range of the nuclear force, nuclear saturation, and the finite surface thickness of real nuclei;

- The Coulomb energy is calculated for a charge distribution with a realistic surface diffuseness;

- The rotational moments of inertia are calculated for rigidly rotating nuclei with realistic surface density profiles;

- The parameters of the model for the various contributions to the energy of the nucleus provide a better fit than do those of the liquid-drop model to nuclear groundstate masses and fission barriers of non-rotating nuclei; 
- A flexible shape parametrization is used which allows accurate estimation of the convergence of results as a function of the number of degrees of freedom of the nuclear shapes considered;

- Accurate modeling of the triaxial nuclear shapes occurs; and

- A precise calculation is made of the value, slope, and curvature of the potentialenergy surface by means of numerical quadrature.

With this model, the properties of points of equilibrium corresponding to nuclear ground states and fission saddle points can be calculated. The results are qualitatively similar to those of the rotating-liquid-drop model, but there are significant quantitative differences in fission-barrier heights and moments of inertia.

Insertion of Yukawa-plus-exponential finite-range macroscopic model [76] has led to lower fission barriers in RFRM. This decrease in barriers is the result of the strong attraction between the surfaces of the two nascent fragments at the saddle point which are about to separate $[77,78]$. Using RFRM, the calculated data are consistent with the experimental value of fission-barrier heights and thus, scaling factors are no longer required.

To study heavy-ion induced nuclear reactions using CRISP model, we have implemented RFRM to calculate the angular momentum dependent fission barrier, $B_{f}(A, Z, L)$, as a function of mass $(A)$ and charge $(Z)$ number and angular momentum $(L)$ [55]:

$$
B_{f}(A, Z, L)=B_{f}^{0}(A, Z) \times h(A, Z, L),
$$

where $B_{f}^{0}(A, Z)$ is the fission barrier at $L=0$ and:

$$
h(A, Z, L)= \begin{cases}1+\delta_{2} L^{2}+\delta_{3} L^{3} & : L \leq L_{20} \\ 1+\gamma_{2} l^{2}+\gamma_{3} l^{3}+\gamma_{4} l^{4}+\gamma_{5} l^{5} & : L>L_{20}\end{cases}
$$

where $l=\frac{L}{L_{\max }}, L_{\max }$ is the value of $L$ where the barrier disappears, $L_{20}$ is the value of $L$ where calculated barrier height is $20 \%$ of $B_{f}^{0}(A, Z)$, and $L_{80}$ is the value of $L$ where calculated barrier height is $80 \%$ of $B_{f}^{0}(A, Z)$. The four quantities $B_{f}^{0}(A, Z), L_{20}, L_{80}$ 
and $L_{\max }$ are approximated by fitting to calculated values functions of the form:

$$
p_{m}(A, Z)=\sum_{i=0}^{N_{A}-1} \sum_{j=0}^{N_{Z}-1} C_{i j}^{(m)} P_{i}(\eta) P_{j}(\mu)
$$

where $p_{m}$ represents $B_{f}^{0}(A, Z), L_{20}, L_{80}$ or $L_{\max } ; \eta=A / 400 ; \mu=Z / 100 ; P_{k}$ is an ordinary Legendre polynomial; and $N_{A}$ and $N_{Z}$ are the number of terms in the resulting $A$ and $Z$ series for fixed $Z$ and $A$, respectively. The values of $\delta_{i}$ and $\gamma_{i}$ are expressible in terms of the last three of these $p_{m}$ 's [55].

To calculate the angular momentum dependent fission barrier using RFRM model, a FORTRAN 77 computer subroutine called BARFIT is written by Sierk [55] which calculates the height of the barrier and the energy of the rotating ground state for given values of $Z, A$ and $L$. To be able to insert this code in CRISP, I have converted it from Fortran 77 to $\mathrm{C}++$ language, made it compatible with CRISP and implemented it in CRISP code. The converted BARFIT code into $\mathrm{C}++$ can be found in Appendix A.

\subsubsection{Extension of RFRM with Nix model}

In BARFIT subroutine code, fission barriers at $L=0$ are calculated from a $7^{\text {th }}$ order fit in two variables for $Z$ values from 20 to 110 , resulting to 638 fitted barriers. These 638 barriers are fit with an RMS deviation $0.10 \mathrm{MeV}$ by this 49-parameter function.

Although the results of this code for angular momentum dependent fission barrier are relatively reliable, to apply a nuclear physics model instead of using fitted values of Sierk's code, as the next step of refining fission-barrier height in CRISP model, instead of using the fitted values of BARFIT for $B_{f}^{0}(A, Z)$, I implemented the Nix model for $B_{f}^{0}(A, Z)$ and merged it to Eq. (4.12).

Fission-barrier height at zero angular momentum calculated by the Nix formula [79] has the form:

$$
B_{f}^{0}(A, Z)=a_{s} \times\left[1-k\left(\frac{N-Z}{A}\right)^{2}\right] \times A^{2 / 3} F(x)
$$


where $a_{s}=17.9439 \mathrm{MeV}, k=1.7826$, and $N=A-Z$. The function $F(x)$ with:

$$
x=\frac{Z^{2} / A}{p \times\left\{1-k[(N-Z) / A]^{2}\right\}},
$$

and $p=50.88$ is defined in Ref. [79]. By this work, I was able to interpret the experimental data within the theoretical framework of rotating liquid drop model by inserting RFRM and Nix models into CRISP. Results of CRISP and its comparison with experimental data are discussed in chapter 5 . 


\section{Chapter 5}

\section{Results and Discussion}

In this work, we have studied the reaction of ${ }^{11} \mathrm{~B}$ ions with ${ }^{181} \mathrm{Ta}$ target at 245.4 and 125.7 MeV, with ${ }^{197} A u$ target at 255.5 and $137.5 \mathrm{MeV}$ and with ${ }^{209} \mathrm{Bi}$ target at $146 \mathrm{MeV}$ energy, and also the reaction of ${ }^{7} \mathrm{Li}$ ions with ${ }^{208} \mathrm{~Pb}$ target at $245 \mathrm{MeV}$ energy. The results of CRISP model are compared with the experimental data measured at the LNR Phasotron (JINR, Dubna, Russia) and for ${ }^{181} \mathrm{Ta}$ and ${ }^{209} \mathrm{Bi}$ taken from Ref. [1].

\subsection{Mass-yield distribution}

The mass-yield distribution determined for the reactions induced by heavy-ion projectiles at intermediate energies are presented in Figs. 5.1, 5.2 and 5.3, with inclusion the fission product range, spallation and the light fragment production on either side. From these figures, one can see the clear-cut distinction between spallation or deep spallation and fission mass ranges.

The CRISP results before applying any modification in fission-barrier height, when only fission barrier at zero angular momentum $\left(B_{f}^{0}(A, Z)\right)$ was performed are shown in Figs. 5.1a, c, 5.2a, c and 5.3a, c. And the results of CRISP after inclusion of angular momentum concept for the heavy-ion induced collisions based on RFRM and Nix model are shown in Figs. 5.1b, d, 5.2b, d and 5.3b, d.

A large hump consisting of the heavy residues in the mass range close to the target mass can be observed. And the processes connected to the production of such nuclei would be spallation or deep spallation. The region of light nuclides with mass $A<40$ 


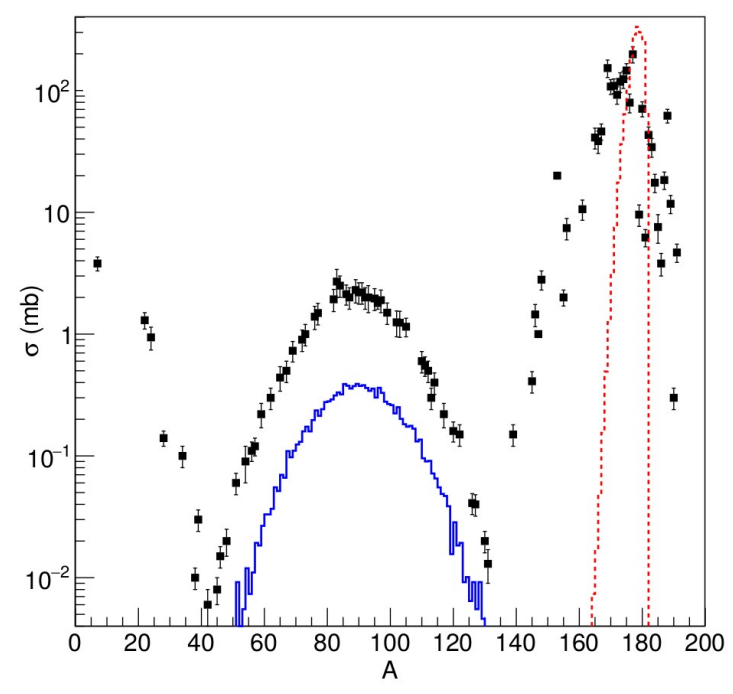

(a) ${ }^{181} \mathrm{Ta}+245.4 \mathrm{MeV}^{11} B$ with $B_{f}^{0}(A, Z)$

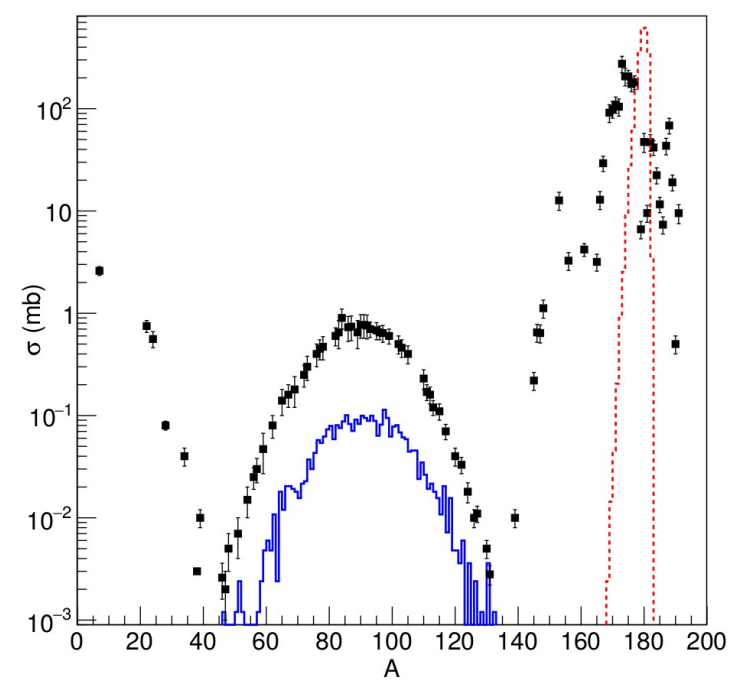

(c) ${ }^{181} \mathrm{Ta}+125.7 \mathrm{MeV}^{11} B$ with $B_{f}^{0}(A, Z)$

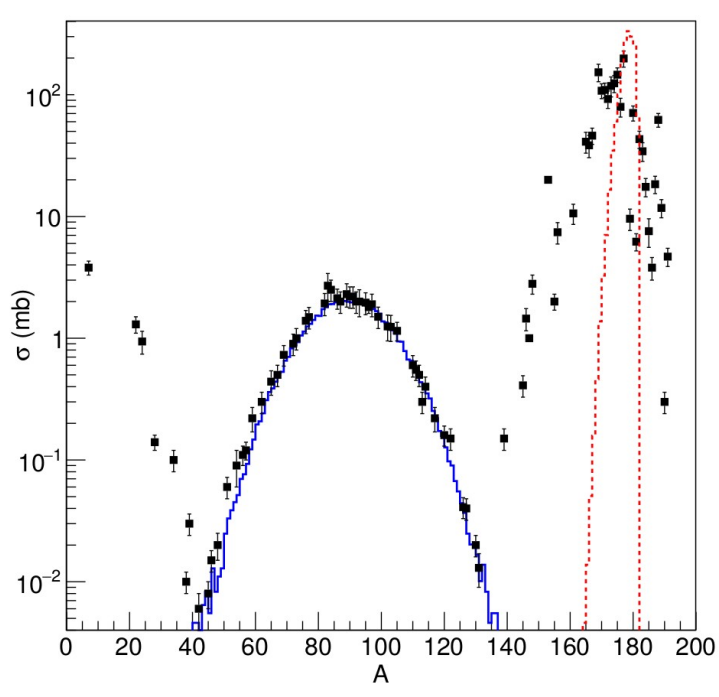

(b) ${ }^{181} \mathrm{Ta}+245.4 \mathrm{MeV}^{11} \mathrm{~B}$ with RFRM + Nix

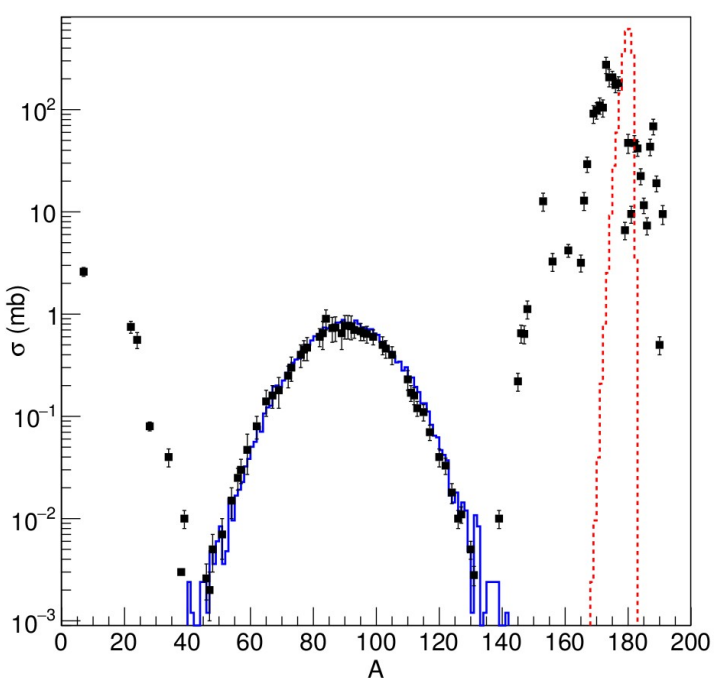

(d) ${ }^{181} \mathrm{Ta}+125.7 \mathrm{MeV}{ }^{11} \mathrm{~B}$ with $\mathrm{RFRM}+\mathrm{Nix}$

Figure 5.1: Mass-yield distributions for the reaction of ${ }^{11} \mathrm{~B}$ ions with ${ }^{181} \mathrm{Ta}$ target at 245.4 and 125.7 MeV energy. The blue solid line indicates the results of the fission cross sections as a function of fragment mass $A$ and the red dotted line corresponds to the spallation cross section, both calculated by the CRISP code, (a) and (c) without any modification in CRISP with the fission barrier at $L=0$, and (b) and (d) after inserting RFRM and Nix models. The experimental data (solid squares) are taken from Ref. [1]. 


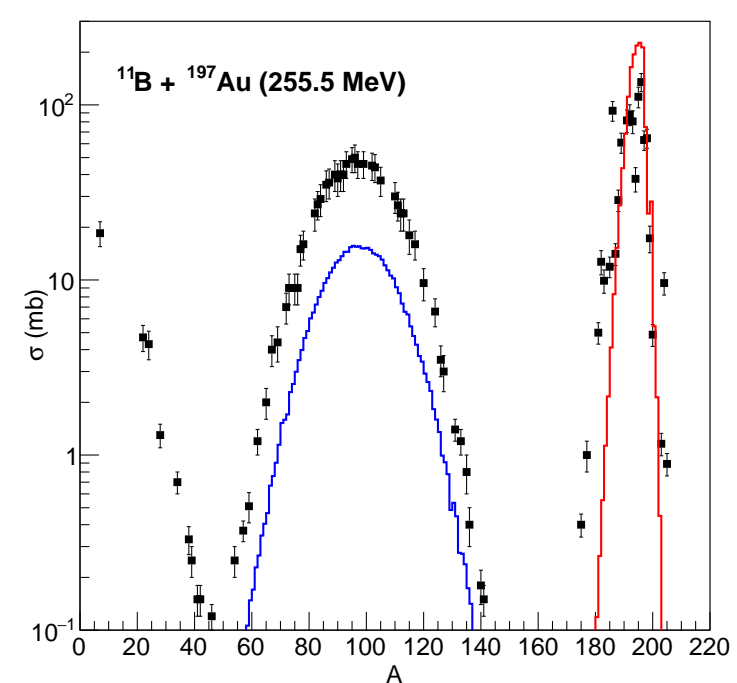

(a) ${ }^{197} A u+255.5 \mathrm{MeV}^{11} B$ with $B_{f}^{0}(A, Z)$

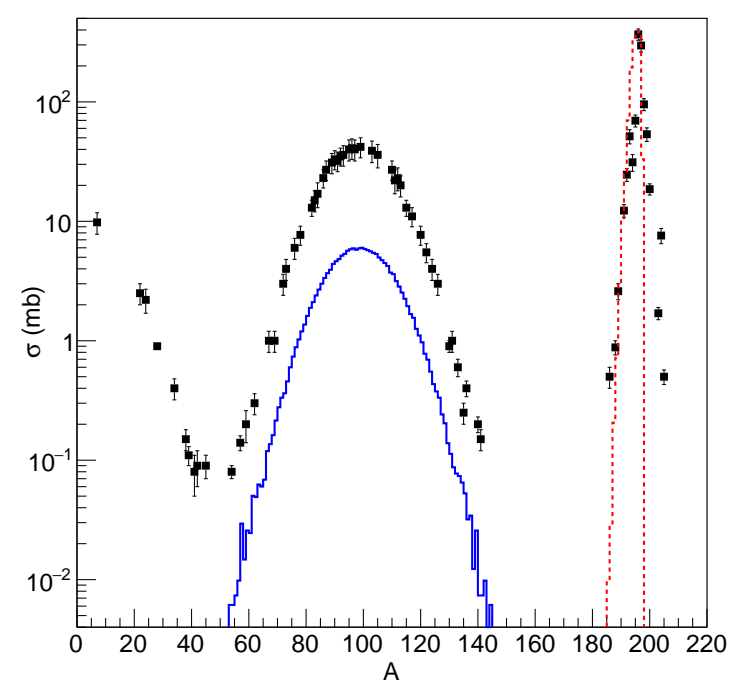

(c) ${ }^{197} A u+137.5 \mathrm{MeV}{ }^{11} B$ with $B_{f}^{0}(A, Z)$

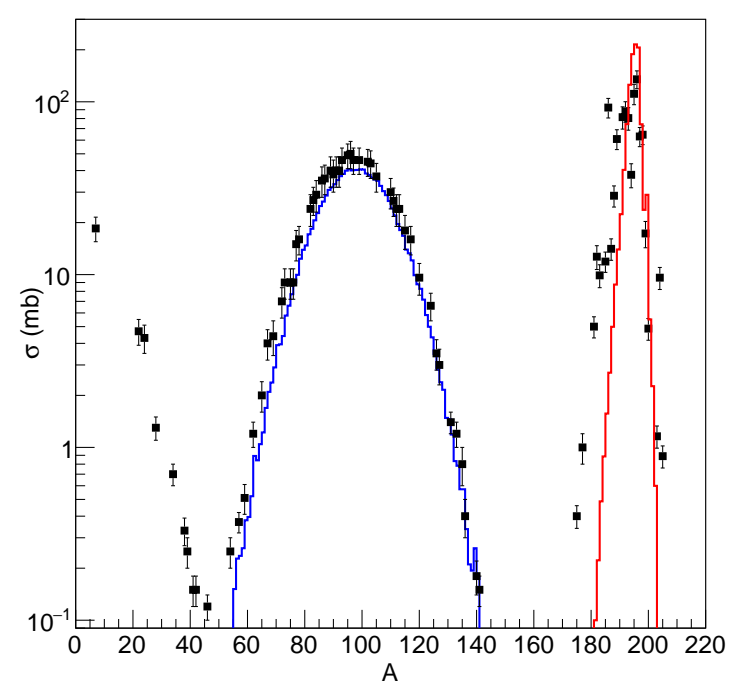

(b) ${ }^{197} A u+255.5 \mathrm{MeV}{ }^{11} B$ with RFRM + Nix

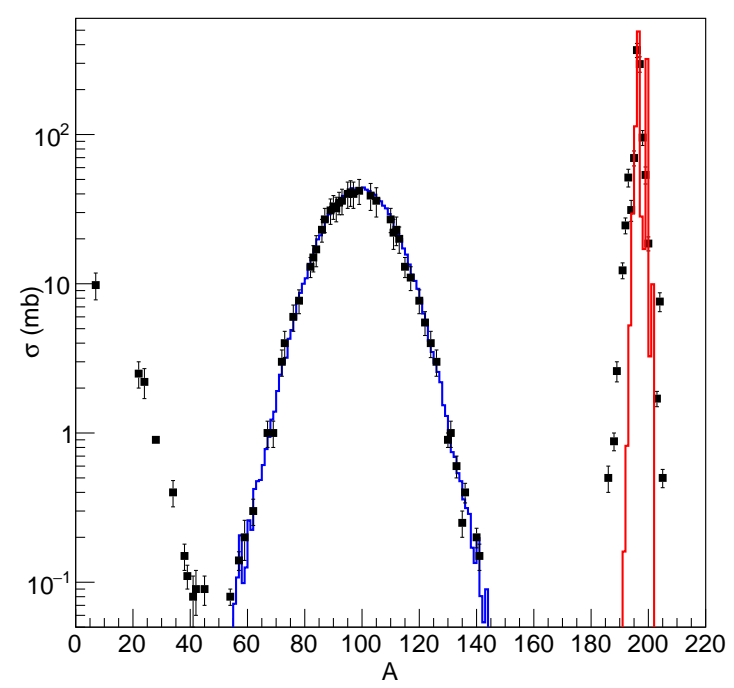

(d) ${ }^{197} A u+137.5 \mathrm{MeV}^{11} B$ with $\mathrm{RFRM}+\mathrm{Nix}$

Figure 5.2: Mass-yield distributions for the reaction of ${ }^{11} B$ ions with ${ }^{197} A u$ target at 255.5 and 137.5 MeV energy. The blue solid line indicates the results of the fission cross sections as a function of fragment mass $A$ and the red dotted line corresponds to the spallation cross section, both calculated by the CRISP code, (a) and (c) without any modification in CRISP with the fission barrier at $L=0$, and (b) and (d) after inserting RFRM and Nix models. 


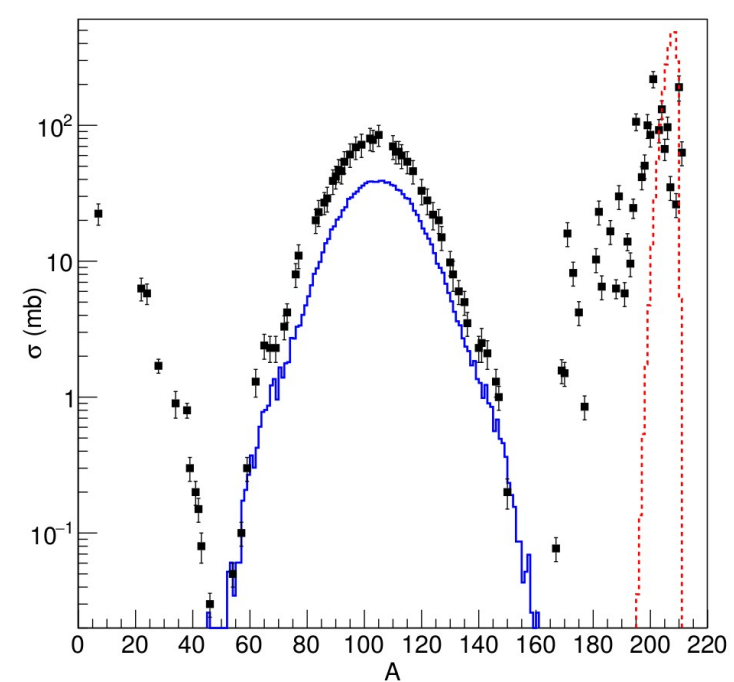

(a) ${ }^{209} B i+146 \mathrm{MeV}^{11} B$ with $B_{f}^{0}(A, Z)$

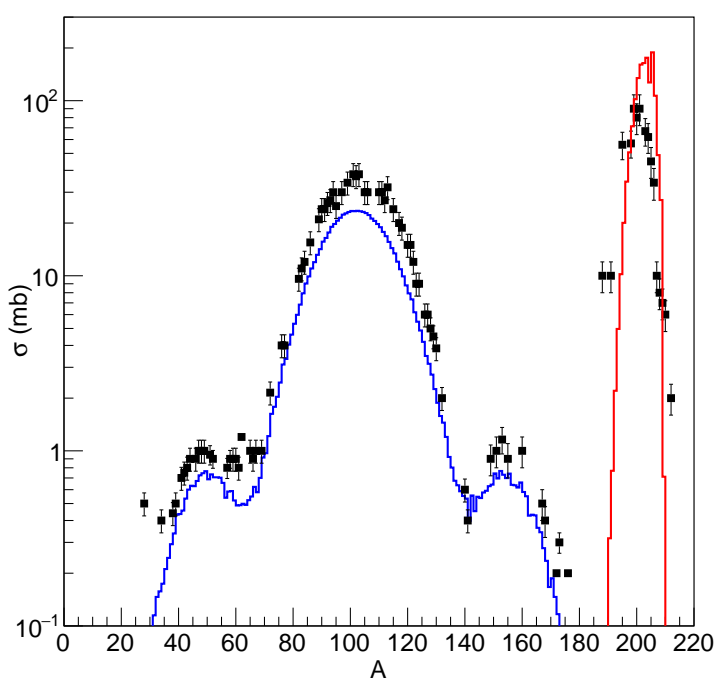

(c) ${ }^{208} \mathrm{~Pb}+245 \mathrm{MeV}^{7} \mathrm{Li}$ with $B_{f}^{0}(A, Z)$

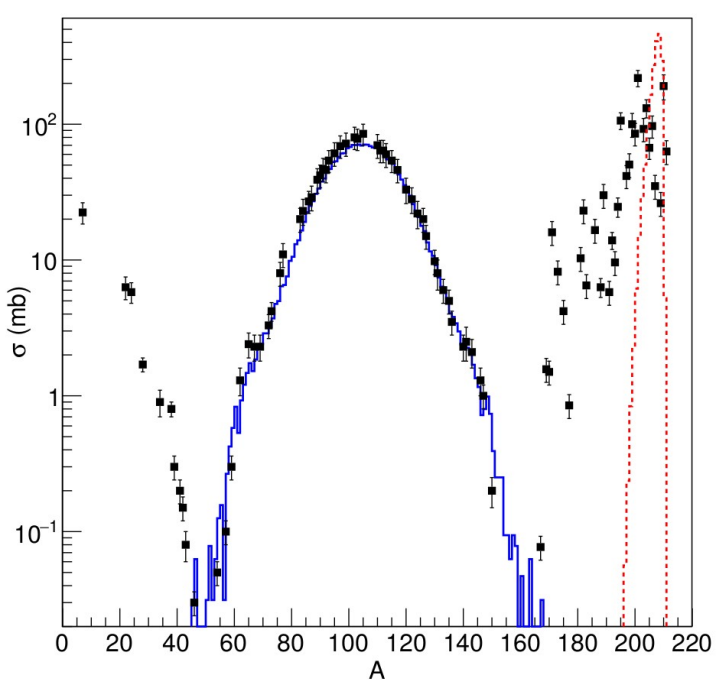

(b) ${ }^{209} \mathrm{Bi}+146 \mathrm{MeV}{ }^{11} B$ with $\mathrm{RFRM}+\mathrm{Nix}$

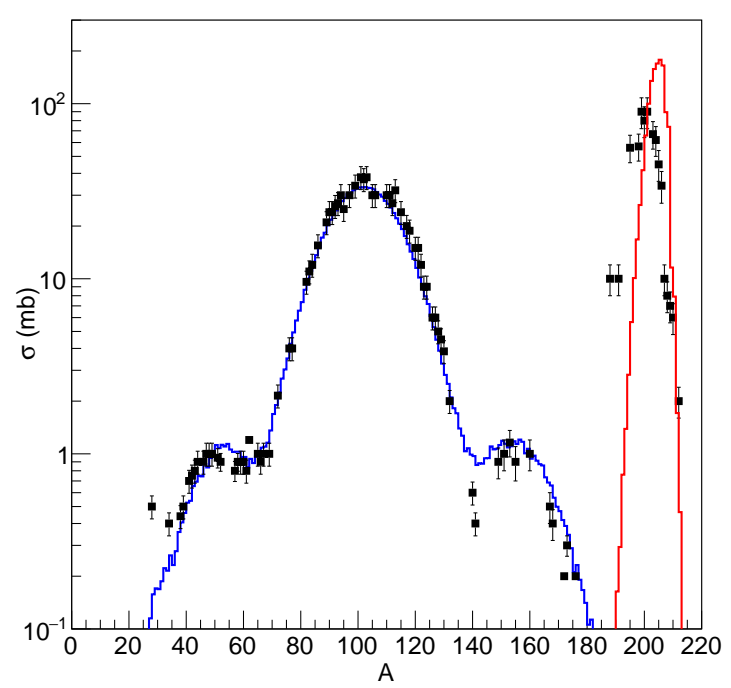

(d) ${ }^{208} \mathrm{~Pb}+245 \mathrm{MeV}{ }^{7} \mathrm{Li}$ with RFRM + Nix

Figure 5.3: Mass-yield distributions for the reaction of ${ }^{11} \mathrm{~B}$ ions with ${ }^{209} \mathrm{Bi}$ target at $146 \mathrm{MeV}$ energy and the reaction of ${ }^{7} \mathrm{Li}$ ions with ${ }^{208} \mathrm{~Pb}$ target at $245 \mathrm{MeV}$ energy. The blue solid line indicates the results of the fission cross sections as a function of fragment mass $A$ and the red dotted line corresponds to the spallation cross section, both calculated by the CRISP code, (a) and (c) without any modification in CRISP with the fission barrier at $L=0$, and (b) and (d) after inserting RFRM and Nix models. The experimental data (solid squares) for the reaction of ${ }^{11} B+{ }^{209} \mathrm{Bi}$ at $146 \mathrm{MeV}$ is taken from Ref. [1]. 
$\mathrm{u}$ contains the intermediate mass fragments (IMFs). IMFs are particles with $A>4 \mathrm{u}$ but lighter than fission fragments and can be formed through the following processes: i) they could be the counterpart pair of products in the mass region of $A \sim 140-185 \mathrm{u}$ for ${ }^{181}$ Ta target, $A \sim 180-205$ u for ${ }^{197} A u$ target, $A \sim 170-215$ u for ${ }^{209} B i$ target, or $A \sim 195-215 \mathrm{u}$ for ${ }^{208} \mathrm{~Pb}$ target, due to spallation or deep spallation processes, where the excited nuclei shall evaporate not only nucleons and light charged particles but also heavier particles in the IMF region; ii) according to the intranuclear cascade model [80], IMFs could be produced by the disintegration of highly excited residual heavy nuclei after evaporation of some nucleons. As can be seen from Figs. 5.1, 5.2 and 5.3, CRISP model cannot reproduce the IMF region yet.

The major region in mass-yield distribution is at the mass range of $A=40-135$ $\mathrm{u}$ for ${ }^{181} \mathrm{Ta}$ target, $A=55-145 \mathrm{u}$ for ${ }^{197} \mathrm{Au}, \mathrm{A}=45-165 \mathrm{u}$ for ${ }^{209} \mathrm{Bi}$ and $A=$ $40-180 \mathrm{u}$ for ${ }^{208} \mathrm{~Pb}$ which are the results of binary fissions. The mass and energy distributions of fission fragments in this area are caused by the valley structure of the deformation potential energy surface of a fissioning nucleus, successfully described by MM-RNR model [20]. The same analysis is also done with other reactions [35] at high energies where the shoulders of asymmetry were more obvious.

As explained in Sec. 4.3, CRISP model uses Eq. (4.12) to consider the dependency of compound nucleus fission barrier on angular momentum (RFRM). Regarding Fig. 4.2, this dependency decreases the fission barrier height and according to Eq. (3.6), it increases the fission cross section. The final effect of considering angular momentum dependent fission barrier on the fission cross section is shown in Figs. 5.1, 5.2 and 5.3. The significant difference in the fission cross sections (blue lines) in the left and right columns of Figs. 5.1, 5.2 and 5.3 is a result of the decrease in fission barrier height as a consequence of angular momentum. This result illustrates how the transferred angular momenta of the reactions increase the fission cross sections due to the decrease of fission barrier heights.

The CRISP results of fission cross sections are achieved with the parameters shown in Tables 5.1, 5.2 and 5.3 using the multimodal fission model. Although very subtle, it is possible to see small shoulders in the experimental data of ${ }^{11} B+{ }^{197} A u$ reaction at 137.5 MeV (asymmetric fission), but not for the reactions of ${ }^{11} B+{ }^{197} A u$ at $255.5 \mathrm{MeV}$ and ${ }^{11} B+{ }^{181} \mathrm{Ta}$ at 125.7 and $245.4 \mathrm{MeV}$ energies (symmetric fission). The shoulders of 
Table 5.1: Parameter values of Eq. (3.13) used to determine experimental and calculated mass distributions; and fission $\left(\sigma_{f}\right)$ and total $\left(\sigma_{t o t}\right)$ cross sections of ${ }^{11} B+{ }^{181} \mathrm{Ta}$ reaction at 245.4 and 125.7 MeV energies.

\begin{tabular}{lccccc}
\hline \hline \multirow{2}{*}{ Parameter } & \multicolumn{2}{c}{${ }^{11} \boldsymbol{B}+{ }^{181} \boldsymbol{T a}(\mathbf{2 4 5 . 4} \mathbf{~ M e V})$} & & \multicolumn{2}{c}{$\boldsymbol{B}+{ }^{181} \boldsymbol{T a}(\mathbf{1 2 5 . 7} \mathbf{~ M e V})$} \\
\cline { 2 - 3 } \cline { 5 - 6 } & Experiment & CRISP & & Experiment & CRISP \\
\hline$K_{S}$ & $73.15 \pm 3.7$ & 73.0 & & $24.0 \pm 1.2$ & 24.0 \\
$\Gamma_{S}$ & $13.3 \pm 0.51$ & 13.5 & & $12.61 \pm 0.48$ & 12.8 \\
$K_{A S}$ & - & - & - & - & - \\
$\Gamma_{A S}$ & - & - & & - & - \\
$D_{A S}$ & - & & & & - \\
$\sigma_{f}(\mathrm{mb})$ & $35.8 \pm 5.4$ & 33.66 & & $12.1 \pm 1.8$ & 13.08 \\
$\sigma_{t o t}(\mathrm{mb})$ & $1981.0 \pm 300.0$ & 1687.4 & & $1875.0 \pm 280.0$ & 2175.17 \\
\hline \hline
\end{tabular}

asymmetry are more obvious in the reactions of ${ }^{11} \mathrm{~B}+{ }^{209} \mathrm{Bi}$ at $146 \mathrm{MeV}$ and ${ }^{7} \mathrm{Li}+{ }^{208} \mathrm{~Pb}$ at $245 \mathrm{MeV}$ energy. Hence, in CRISP model, the fission cross section as a function of mass number in the reaction of $146 \mathrm{MeV}^{11} \mathrm{~B}$ ions with ${ }^{209} \mathrm{Bi}, 137.5 \mathrm{MeV}^{11} \mathrm{~B}$ ions with ${ }^{197} \mathrm{Au}$ and $245 \mathrm{MeV}^{7} \mathrm{Li}$ ions with ${ }^{208} \mathrm{~Pb}$ target are well described by the sum of three Gaussian functions, corresponding to symmetric and asymmetric fission modes (Eq. (3.13)), but in the reactions of ${ }^{11} \mathrm{~B}$ ions with ${ }^{181} \mathrm{Ta}$ target at 245.4 and $125.7 \mathrm{MeV}$ energies and with ${ }^{197} \mathrm{Au}$ at $255.5 \mathrm{MeV}$, only the symmetric fission component of the mass distribution is considered. The experimental total reaction cross section is computed by summing the cross section of fission, spallation and light nuclide production. The total reaction cross sections calculated by CRISP are also tabulated in Tables 5.1, 5.2 and 5.3 summing the fission and spallation cross sections. The mass-yield distributions calculated by the CRISP code reproduce the shape of the experimental distributions. The total fission cross sections computed by CRISP are also in a good agreement with the experimental ones.

In addition to the fission, we calculated the spallation products mass distribution through the spallation process in which only one heavy fragment with a mass close to the target mass is formed. The results are shown in Figs. 5.1, 5.2 and 5.3, where we observe that at higher mass numbers, the CRISP computations show a fair agreement with 
Table 5.2: Parameter values of Eq. (3.13) used to determine experimental and calculated mass distributions; and fission $\left(\sigma_{f}\right)$ and total $\left(\sigma_{t o t}\right)$ cross sections of ${ }^{11} B+{ }^{197} A u$ reaction at 255.5 and 137.5 MeV energies.

\begin{tabular}{lccccc}
\hline \hline Parameter & \multicolumn{2}{c}{${ }^{11} \boldsymbol{B}+{ }^{197} \boldsymbol{A} \boldsymbol{u}(\mathbf{2 5 5 . 5} \mathbf{M e V})$} & & \multicolumn{2}{c}{$\boldsymbol{B}+{ }^{197} \boldsymbol{A u}(\mathbf{1 3 7 . 5} \mathbf{M e V})$} \\
\cline { 2 - 3 } \cline { 6 - 6 } & Experiment & CRISP & & Experiment & CRISP \\
\hline$K_{S}$ & $308.75 \pm 15.1$ & 308.0 & & $119.7 \pm 11.2$ & 250.0 \\
$\Gamma_{S}$ & $12.61 \pm 0.89$ & 12.5 & & $11.57 \pm 0.67$ & 11.5 \\
$K_{A S}$ & - & - & & $1.77 \pm 0.02$ & 0.1 \\
$\Gamma_{A S}$ & - & - & & $2.8 \pm 0.1$ & 2.8 \\
$D_{A S}$ & - & - & & $40.0 \pm 1.6$ & 40.0 \\
$\sigma_{f}(\mathrm{mb})$ & $785.0 \pm 118.0$ & 633.17 & & $601.8 \pm 90.3$ & 626.2 \\
$\sigma_{t o t}(\mathrm{mb})$ & $1749.0 \pm 263.0$ & 1674.6 & & $1651.02 \pm 248.0$ & 1645.4 \\
\hline \hline
\end{tabular}

Table 5.3: Parameter values of Eq. (3.13) used to determine experimental and calculated mass distributions; and fission $\left(\sigma_{f}\right)$ and total $\left(\sigma_{t o t}\right)$ cross sections of ${ }^{7} \mathrm{Li}+{ }^{208} \mathrm{~Pb}$ reaction at $245 \mathrm{MeV}$ and ${ }^{11} B+{ }^{209} B i$ reaction at $146 \mathrm{MeV}$ energy.

\begin{tabular}{lccccc}
\hline \hline \multirow{2}{*}{ Parameter } & \multicolumn{2}{c}{${ }^{7} \boldsymbol{L} \boldsymbol{i}+{ }^{208} \boldsymbol{P b}(\mathbf{2 4 5} \mathbf{M e V})$} & & \multicolumn{2}{c}{$\boldsymbol{1 1} \boldsymbol{B}+{ }^{209} \boldsymbol{B i}(\mathbf{1 4 6} \mathbf{M e V})$} \\
\cline { 2 - 3 } \cline { 5 - 6 } & Experiment & CRISP & & Experiment & CRISP \\
\hline$K_{S}$ & 1220.0 & 1215 & & $177.0 \pm 5.9$ & 730.0 \\
$\Gamma_{S}$ & 12.74 & 12.72 & & $12.90 \pm 0.50$ & 12.7 \\
$K_{A S}$ & 0.048 & 28 & & $8.60 \pm 0.09$ & 5.0 \\
$\Gamma_{A S}$ & 10.0 & 9.5 & & $2.86 \pm 0.11$ & 6.0 \\
$D_{A S}$ & 52.5 & 53.5 & & $39.0 \pm 1.5$ & 37.5 \\
$\sigma_{f}(\mathrm{mb})$ & $634.6 \pm 95.0$ & 557.9 & & $1263.0 \pm 189.0$ & 1135.16 \\
$\sigma_{t o t}(\mathrm{mb})$ & $1118.4 \pm 167.9$ & 1820 & & $2754.0 \pm 413.0$ & 2934.72 \\
\hline \hline
\end{tabular}


the experimental data in the spallation region. While as the spallation product mass number decreases, the CRISP model calculations take distance from the experimental results, which shows that CRISP still needs to be modified for spallation process.

Also comparison between the left and right columns of Figs. 5.1, 5.2 and 5.3 confirms that for heavy-ion induced fusion-fission reactions, the fission barrier is dependent on the angular momentum, and by the increase of projectile energy, the transferred angular momentum increases and as a result, the fission barrier decreases which consequently results in the increase of fission cross section. As aforementioned, BARFIT code uses fitting method to calculate fission-barrier height at zero angular momentum, $B_{f}^{0}(A, Z)$. To have a more precise calculation, we used the Nix model to compute $B_{f}^{0}(A, Z)$ and merged it with BARFIT code and implemented in CRISP model. As it is obvious from Figs. 5.1, 5.2 and 5.3, the angular momentum dependent fission barrier height, $B_{f}(A, Z, L)$, and accordingly the fission cross section are well reproduced in the rotating liquid drop framework by applying RFRM and Nix model in the CRISP code leading to an acceptable explanation of experimental data by CRISP model.

\subsection{Pre- and post-scission nucleons}

The Monte Carlo CRISP code simulates the entire process of a nuclear reaction from the first interaction of projectile with target nucleus up to the production of fission fragments and spallation. When a nucleus fissions, two unstable fission fragments are produced which can evaporate by the Weisskopf statistical evaporation model. At the end, the final fission and spallation products shall be compared to the experimental data. Therefore, contrary to experiment, using CRISP model, we can calculate fissioning nuclei $\left(A_{f}\right)$ at the moment of fissioning, and the summation of binary-fission fragments $\left(A_{f f}\right)$ after postscission evaporations, where $A_{H}+A_{L}=A_{f f}$ and $A_{S}=A_{f f} / 2$.

Pre-scission nucleons are emitted during the cascade and after the formation of equilibrated compound nucleus at the evaporation-fission competition. After the system approaches the scission point, post-scission nucleons are emitted by evaporation from the primary fragments to the fission products. With the CRISP model, we are able to calculate the average number of pre- and post-scission emitted neutrons and protons, separately 
Table 5.4: Mean mass number $\left(\bar{A}_{S}\right)$; mean mass of the fissioning nucleus $\left(\bar{A}_{f}\right)$ after evaporation of pre-scission nucleons from the compound nucleus; mean mass of the summation of binary-fission fragments $\left(\bar{A}_{f f}\right)$ after evaporation of post-scission nucleons from the fragments; average number of pre-scission neutrons $\left(\nu_{\text {pre_n }}\right)$, pre-scission protons $\left(\nu_{\text {pre_p }}\right)$, post-scission neutrons $\left(\nu_{\text {post_n }}\right)$, and post-scission protons $\left(\nu_{\text {post_p }}\right)$; and the average number of total emitted nucleons $\left(\nu_{T}\right)$ of ${ }^{11} \mathrm{~B}+{ }^{181} \mathrm{Ta}$ reaction at 245.4 and $125.7 \mathrm{MeV}$ energies.

\begin{tabular}{lccccc}
\hline \hline \multirow{2}{*}{ Parameter } & \multicolumn{2}{c}{${ }^{11} \boldsymbol{B}+{ }^{181} \mathbf{T a}(\mathbf{2 4 5 . 4} \mathbf{~ M e V})$} & & \multicolumn{2}{c}{$\boldsymbol{B}+{ }^{181} \mathbf{T a}(\mathbf{1 2 5 . 7} \mathbf{~ M e V})$} \\
\cline { 2 - 3 } \cline { 6 - 6 } & Experiment & CRISP & & Experiment & CRISP \\
\hline $\bar{A}_{S}$ & $88.7 \pm 0.6$ & 89.07 & & $90.5 \pm 0.3$ & 90.06 \\
$\bar{A}_{f}$ & - & 179.9 & & - & 181.5 \\
$\bar{A}_{f f}$ & $177.4 \pm 1.2$ & 178.14 & & $181.0 \pm 0.6$ & 180.12 \\
$\nu_{\text {pre_n }}$ & - & 10.09 & & - & 8.42 \\
$\nu_{\text {pre_p }}$ & - & 2.01 & & - & 2.08 \\
$\nu_{\text {post_n }}$ & - & 1.75 & & - & 1.38 \\
$\nu_{\text {post_p }}$ & - & 0.01 & & - & 0 \\
$\nu_{T}$ & $14.6 \pm 2.0$ & 13.86 & & $11.0 \pm 1.5$ & 11.88 \\
\hline \hline
\end{tabular}

(reported in Tables 5.4, 5.5 and 5.6). The comparison between the pre- and post-scission nucleons shows that the average number of pre-scission nucleons is higher than the average number of post-scission ones indicating that the pre-scission evaporation chain is longer. Besides, the neutron evaporation of the systems indicates a large contribution for the production of neutron-deficient nuclides. Also the sum of these contributions gives the average number of total emitted nucleons $\left(\nu_{T}\right)$. This analysis shows that CRISP model describes these experimental data fairly well as a result of well describing the mechanism of the nucleons emission in the pre- and post-scission stages of the nuclear reaction (also shown in Tables 5.4, 5.5 and 5.6). 
Table 5.5: Mean mass number $\left(\bar{A}_{S}\right)$; mean mass of the fissioning nucleus $\left(\bar{A}_{f}\right)$ after evaporation of pre-scission nucleons from the compound nucleus; mean mass of the summation of binary-fission fragments $\left(\bar{A}_{f f}\right)$ after evaporation of post-scission nucleons from the fragments; average number of pre-scission neutrons $\left(\nu_{\text {pre_n }}\right)$, pre-scission protons $\left(\nu_{\text {pre_p }}\right)$, post-scission neutrons $\left(\nu_{\text {post_n }}\right)$, and post-scission protons $\left(\nu_{\text {post_p }}\right)$; and the average number of total emitted nucleons $\left(\nu_{T}\right)$ of ${ }^{11} B+{ }^{197} A u$ reaction at 255.5 and $137.5 \mathrm{MeV}$ energies.

\begin{tabular}{lccccc}
\hline \hline \multirow{2}{*}{ Parameter } & \multicolumn{2}{c}{${ }^{11} \boldsymbol{B}+{ }^{197} \boldsymbol{A u}(\mathbf{2 5 5 . 5} \mathbf{M e V})$} & & \multicolumn{2}{c}{$\boldsymbol{1 1}+{ }^{197} \boldsymbol{A u}(\mathbf{1 3 7 . 5} \mathbf{M e V})$} \\
\cline { 2 - 3 } \cline { 5 - 6 } & Experiment & CRISP & & Experiment & CRISP \\
\hline $\bar{A}_{S}$ & $99.0 \pm 0.5$ & 98.63 & & $97.0 \pm 0.7$ & 97.48 \\
$\bar{A}_{f}$ & - & 198 & & - & 199.9 \\
$\bar{A}_{f f}$ & $198 \pm 1.0$ & 197.26 & & $194 \pm 1.4$ & 194.96 \\
$\nu_{\text {pre_n }}$ & - & 5.75 & & - & 5.34 \\
$\nu_{\text {pre_p }}$ & - & 4.25 & & - & 2.76 \\
$\nu_{\text {post_n }}$ & - & 0.73 & & - & 4.84 \\
$\nu_{\text {post_p }}$ & - & 0.01 & & - & 0.10 \\
$\nu_{T}$ & $10.0 \pm 1.5$ & 10.74 & & $14.0 \pm 2.1$ & 13.04 \\
\hline \hline
\end{tabular}


Table 5.6: Mean mass number $\left(\bar{A}_{S}\right)$; mean mass of the fissioning nucleus $\left(\bar{A}_{f}\right)$ after evaporation of pre-scission nucleons from the compound nucleus; mean mass of the summation of binary-fission fragments $\left(\bar{A}_{f f}\right)$ after evaporation of post-scission nucleons from the fragments; average number of pre-scission neutrons $\left(\nu_{\text {pre }} n\right)$, pre-scission protons $\left(\nu_{\text {pre_p }}\right)$, post-scission neutrons $\left(\nu_{\text {post_n }}\right)$, and post-scission protons $\left(\nu_{\text {post_p }}\right)$; and the average number of total emitted nucleons $\left(\nu_{T}\right)$ of ${ }^{7} \mathrm{Li}+{ }^{208} \mathrm{~Pb}$ reaction at $245 \mathrm{MeV}$ and ${ }^{11} \mathrm{~B}+{ }^{209} \mathrm{Bi}$ reaction at $146 \mathrm{MeV}$ energy.

\begin{tabular}{|c|c|c|c|c|}
\hline \multirow[t]{2}{*}{ Parameter } & \multicolumn{2}{|c|}{${ }^{7} \mathrm{Li}+{ }^{208} \mathrm{~Pb}(245 \mathrm{MeV})$} & \multicolumn{2}{|c|}{${ }^{11} B+{ }^{209} B i(146 \mathrm{MeV})$} \\
\hline & Experiment & CRISP & Experiment & CRISP \\
\hline $\bar{A}_{S}$ & $103 \pm 0.9$ & 102.1 & $104.0 \pm 1.4$ & 104.3 \\
\hline $\bar{A}_{f}$ & - & 208.7 & - & 212.8 \\
\hline $\bar{A}_{f f}$ & 206 & 204.2 & $208.0 \pm 2.8$ & 208.6 \\
\hline$\nu_{p r e \_n}$ & - & 5.15 & - & 5.59 \\
\hline$\nu_{\text {pre_p }}$ & - & 1.15 & - & 1.61 \\
\hline$\nu_{\text {post_n}}$ & - & 4.31 & - & 4.15 \\
\hline$\nu_{\text {post_p }}$ & - & 0.19 & - & 0.05 \\
\hline$\nu_{T}$ & $9.0 \pm 2.0$ & 10.8 & $12.0 \pm 1.6$ & 11.4 \\
\hline
\end{tabular}




\subsection{Fissility parameter}

Chung et al. [81, 82] showed that it is possible to use an empirical expression to estimate the probability of symmetric and asymmetric fission cross sections. Based on Chung et al.'s studies, there is a close relation between the contribution of symmetric and asymmetric fission modes and the fissility parameter. The empirical formula suggested by Chung et al. [82] for the critical value of the fissility parameter is:

$$
\left(Z_{f}^{2} / A_{f}\right)_{c r .}=35.5+0.4\left(Z_{f}-90\right)
$$

where $Z_{f}$ and $A_{f}$ are the charge and mass number of the fissioning nucleus, respectively. According to Chung et al. [81, 82], for reactions with a higher abundance of fissioning nuclei above the critical fissility parameter, the symmetric fission mode is dominant, while for the predominance of fissioning systems below the critical fissility line, the main fission channel would be asymmetric. The more population of fissioning systems above critical fissility line implies the higher probability of obtaining a symmetric mass distribution.

Comparing fission fragment mass distributions presented in Figs. 5.1, 5.2 and 5.3, we see that for the reactions of ${ }^{11} \mathrm{~B}$ ions on ${ }^{181} \mathrm{Ta}$ target at 245.4 and $125.7 \mathrm{MeV}$ energies and on ${ }^{197} \mathrm{Au}$ target at $255.5 \mathrm{MeV}$, the symmetric fission mode is the only dominant channel, while for the reactions of ${ }^{11} \mathrm{~B}$ ions with ${ }^{197} \mathrm{Au}$ at $137.5 \mathrm{MeV}$ and ${ }^{209} \mathrm{Bi}$ at $146 \mathrm{MeV}$, and ${ }^{7} \mathrm{Li}$ ions with ${ }^{208} \mathrm{~Pb}$ at $245 \mathrm{MeV}$ energy, the asymmetric fission mode is also evident. The contribution of fissioning systems above and below the critical fissility parameter can be analyzed with the help of CRISP model. For each simulation case $i$ in the CRISP code, we calculate the charge $Z_{f}(i)$ and mass $A_{f}(i)$ of the fissioning nucleus, and obtain the fissility paramter $\left(Z_{f}^{2} / A_{f}\right)_{i}$ for that bin. The average mass and charge number of the fissioning systems, and the average and critical fissility parameters are brought in Table 5.7. For the reactions of ${ }^{11} \mathrm{~B}$ ions with ${ }^{209} \mathrm{Bi}$ target at $146 \mathrm{MeV}$ and with ${ }^{197} \mathrm{Au}$ at $137.5 \mathrm{MeV}$ and ${ }^{7} \mathrm{Li}$ ions with ${ }^{208} \mathrm{~Pb}$ at $245 \mathrm{MeV}$, the difference between $Z_{f}^{2} / A_{f}$ and $\left(Z_{f}^{2} / A_{f}\right)_{c r}$. is less than the three other reactions, as a result of the narrow shoulders of asymmetric fission channel; while for the reactions of ${ }^{11} \mathrm{~B}$ ions with ${ }^{181} \mathrm{Ta}$ at 245.4 and $125.7 \mathrm{MeV}$ energies and with ${ }^{197} \mathrm{Au}$ at $255.5 \mathrm{MeV}$, this difference is higher, asserting the pure symmetric fission mode of the three latter reactions. 
Table 5.7: Charge and mass number of the fissioning system and the average fissility parameter $\left(Z_{f}^{2} / A_{f}\right)$ calculated by CRISP for the ${ }^{11} B+{ }^{181} \mathrm{Ta}$ reaction at 245.4 and $125.7 \mathrm{MeV}$ energies, ${ }^{11} \mathrm{~B}+{ }^{197} \mathrm{Au}$ reaction at 255.5 and $137.5 \mathrm{MeV},{ }^{7} \mathrm{Li}+{ }^{208} \mathrm{~Pb}$ reaction at $245 \mathrm{MeV}$ and ${ }^{11} \mathrm{~B}+{ }^{209} \mathrm{Bi}$ reaction at $146 \mathrm{MeV}$ energy. The critical fissility parameter $\left(Z_{f}^{2} / A_{f}\right)_{c r}$. is also presented.

\begin{tabular}{ccccc}
\hline \hline Reaction & Energy $(\mathbf{M e V})$ & $\left(\boldsymbol{Z}_{\boldsymbol{f}}, \boldsymbol{A}_{\boldsymbol{f}}\right)$ & $\boldsymbol{Z}_{\boldsymbol{f}}^{\mathbf{2}} / \boldsymbol{A}_{\boldsymbol{f}}$ & $\left(\boldsymbol{Z}_{\boldsymbol{f}}^{\mathbf{2}} / \boldsymbol{A}_{\boldsymbol{f}}\right)_{\boldsymbol{c}} \mathbf{.}$ \\
\hline${ }^{11} \mathrm{~B}+{ }^{181} \mathrm{Ta}$ & 245.4 & $(76,180)$ & 32.09 & 29.9 \\
${ }^{11} \mathrm{~B}+{ }^{181} \mathrm{Ta}$ & 125.7 & $(76,182)$ & 31.74 & 29.9 \\
${ }^{11} \mathrm{~B}+{ }^{197} \mathrm{Au}$ & 255.5 & $(81,200)$ & 32.8 & 31.9 \\
${ }^{11} \mathrm{~B}+{ }^{197} \mathrm{Au}$ & 137.5 & $(80,198)$ & 32.3 & 31.5 \\
${ }^{7} \mathrm{Li}+{ }^{208} \mathrm{~Pb}$ & 245 & $(84,211)$ & 33.44 & 33.1 \\
${ }^{11} \mathrm{~B}+{ }^{209} \mathrm{Bi}$ & 146 & $(86,213)$ & 34.72 & 33.9 \\
\hline \hline
\end{tabular}

Fig. 5.4 illustrates the distribution of the fissility parameter, $Z_{f}^{2} / A_{f}$, of the fissioning nuclei as a function of $Z_{f}$. Its abundance is also given by the right column, where the darker color denotes the larger counts of fissility parameter in that region and vice versa. As can also be seen from Fig. 5.4, while there is roughly a complete distribution of fissioning systems above the critical fissility line for the reactions of ${ }^{11} \mathrm{~B}+{ }^{181} \mathrm{Ta}$ at 245.4 and $125.7 \mathrm{MeV}$ energies and ${ }^{11} B+{ }^{197} A u$ at $255.5 \mathrm{MeV}$ (due to pure symmetric distribution), a small distribution of fissioning systems below the critical fissility line is obvious for the reactions of ${ }^{11} \mathrm{~B}+{ }^{197} \mathrm{Au}$ at $137.5 \mathrm{MeV},{ }^{7} \mathrm{Li}+{ }^{208} \mathrm{~Pb}$ at $245 \mathrm{MeV}$ and ${ }^{11} \mathrm{~B}+{ }^{209} \mathrm{Bi}$ at $146 \mathrm{MeV}$, affirming the presence of asymmetric fission channel for these reactions. Besides, the most contribution of the fissioning systems for all six reactions are located above the critical fissility line, confirming that the predominant fission mode is symmetric. This is due to the fact that the process of symmetric or asymmetric fission depends on shell effects that decrease in its intensity as the excitation energy of the fissioning system increases. As can be observed in the experimental data and also CRISP results, asymmetric fission is negligible for all cases studied here, indicating that the shell effects can be disregarded in the calculations. 


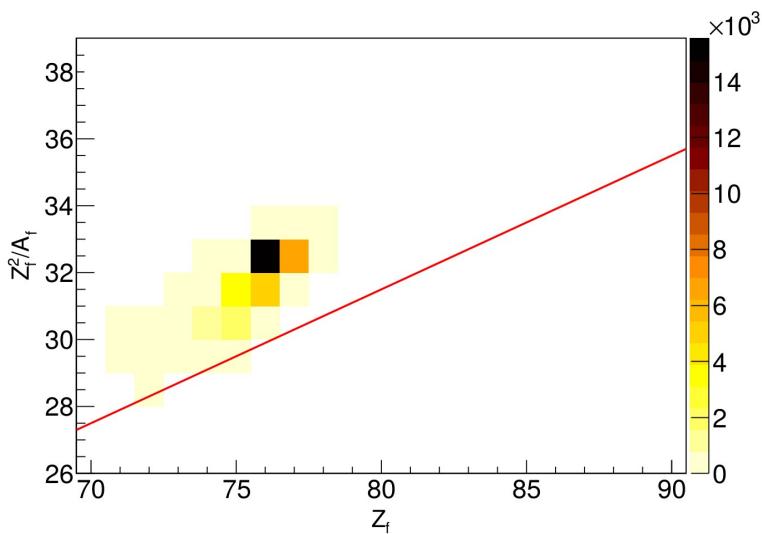

(a) ${ }^{181} \mathrm{Ta}+245.4 \mathrm{MeV}^{11} \mathrm{~B}$

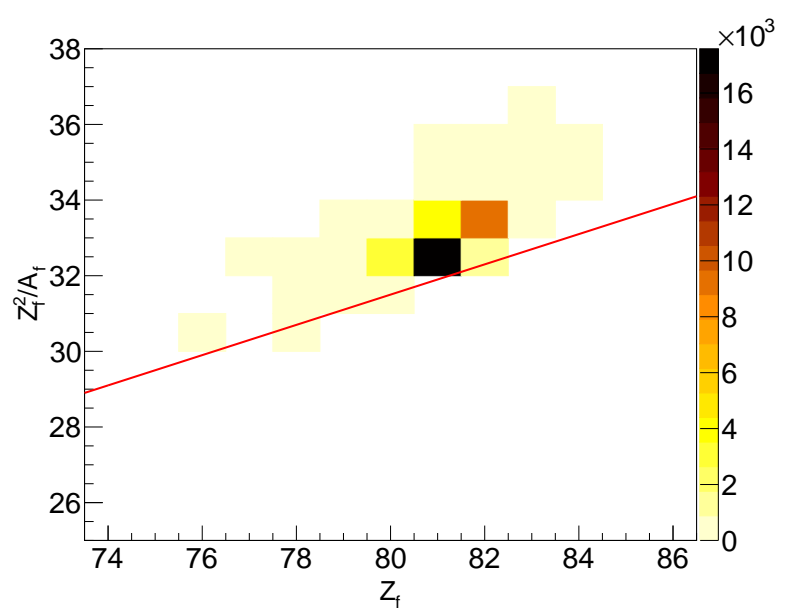

(c) ${ }^{197} \mathrm{Au}+255.5 \mathrm{MeV}^{11} \mathrm{~B}$

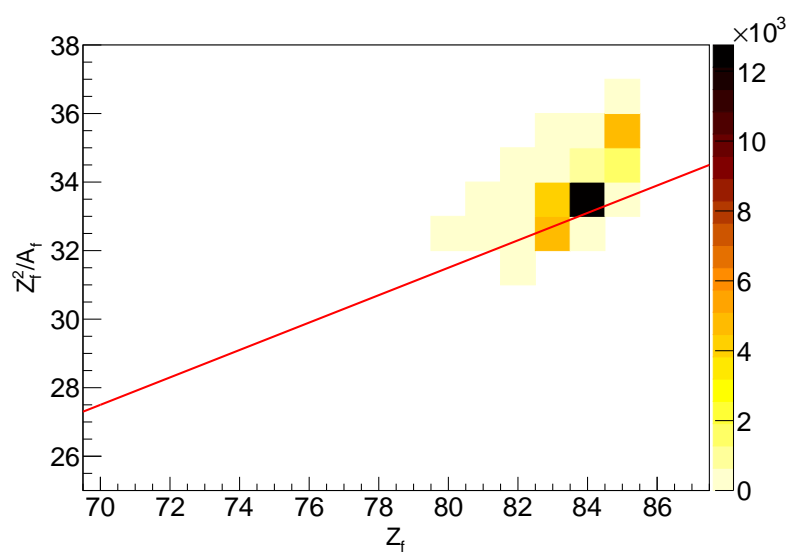

(e) ${ }^{208} \mathrm{~Pb}+245 \mathrm{MeV}^{7} \mathrm{Li}$

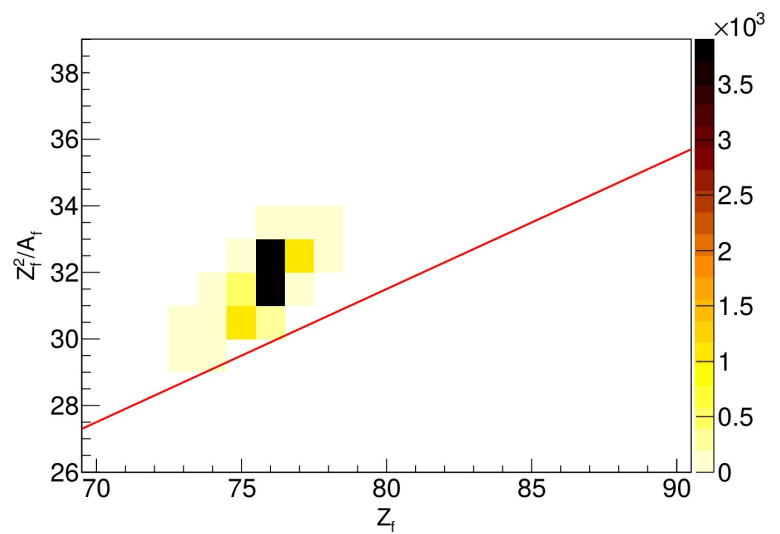

(b) ${ }^{181} \mathrm{Ta}+125.7 \mathrm{MeV}{ }^{11} \mathrm{~B}$

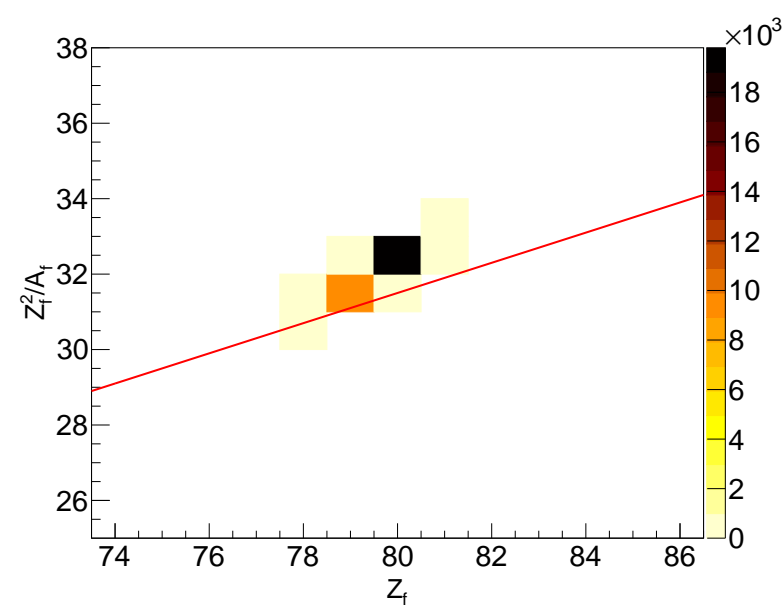

(d) ${ }^{197} \mathrm{Au}+137.5 \mathrm{MeV}^{11} \mathrm{~B}$

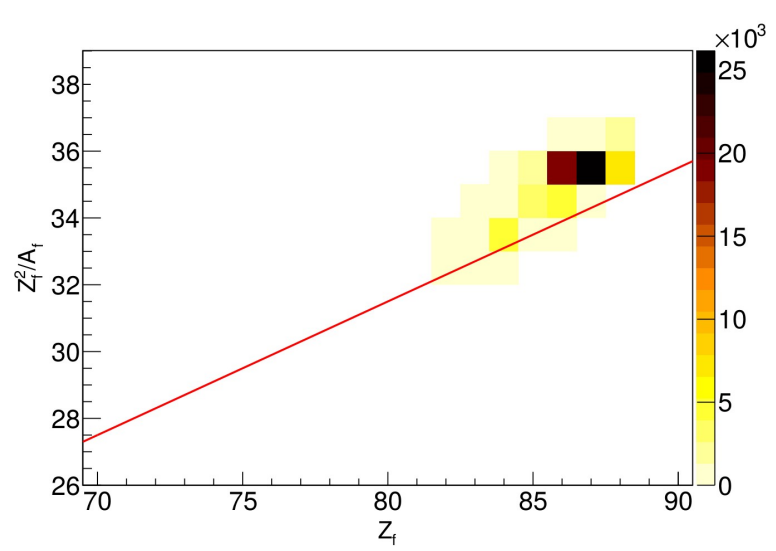

(f) ${ }^{209} \mathrm{Bi}+146 \mathrm{MeV}^{11} \mathrm{~B}$

Figure 5.4: Color map distribution of the fissility parameter $Z_{f}^{2} / A_{f}$ for the fissioning systems of the reactions ${ }^{11} B+{ }^{181} \mathrm{Ta}$ at 245.4 and $125.7 \mathrm{MeV}$ energies, ${ }^{11} \mathrm{~B}+{ }^{197} \mathrm{Au}$ at 255.5 and 137.5 $\mathrm{MeV},{ }^{7} \mathrm{Li}+{ }^{208} \mathrm{~Pb}$ at $245 \mathrm{MeV}$ and ${ }^{11} \mathrm{~B}+{ }^{209} \mathrm{Bi}$ at $146 \mathrm{MeV}$. The solid red line corresponds to the critical fissility parameter as a function of $Z_{f}[81,82]$. 


\subsection{Fissility}

Another interesting parameter which is worth calculating is the fission probability (fissility) derived by the ratio of fission cross section $\left(\sigma_{f}\right)$ to the total reaction cross section $\left(\sigma_{t o t}\right)$. As can be seen in Table 5.8, the calculated values of fissility by CRISP model lie successfully within the uncertainties of the experimental determination, except for the ${ }^{7} \mathrm{Li}+{ }^{208} \mathrm{~Pb}$ reaction where the computed fissility by CRISP has a lower value. Also comparing the fissilities taken by CRISP and experiment for heavy-ion induced collisions with the ones for proton-induced fissions [83] shows that the fission probabilities induced by ${ }^{11} \mathrm{~B}$ ions is about one order higher than the fissilities induced by protons at the same intermediate energy ranges. It confirms that the higher angular momenta which are transferred by heavy-ions to the pre-actinide ${ }^{181} \mathrm{Ta}$ and ${ }^{209} \mathrm{Bi}$ targets lead to higher rotational energies transferred to the compound nuclei which consequently increase the fission cross sections and fissilities (compared to the nucleon-induced collisions). 
Table 5.8: Fissility of the ${ }^{11} B+{ }^{181} \mathrm{Ta}$ and $p+{ }^{181} \mathrm{Ta}$ reactions at 245.4 and $125.7 \mathrm{MeV}$ energies, ${ }^{11} \mathrm{~B}+{ }^{197} \mathrm{Au}$ and $p+{ }^{197} \mathrm{Au}$ reactions at 255.5 and $137.5 \mathrm{MeV}$ energies, ${ }^{7} \mathrm{Li}+{ }^{208} \mathrm{~Pb}$ reaction at $245 \mathrm{MeV}$ and the fission probability of the ${ }^{11} \mathrm{~B}+{ }^{209} \mathrm{Bi}$ and $p+{ }^{209} \mathrm{Bi}$ reactions at $146 \mathrm{MeV}$.

\begin{tabular}{cccc}
\hline \hline Reaction & Energy (MeV) & \multicolumn{2}{c}{ Fissility } \\
\cline { 3 - 4 } & & Experiment & CRISP \\
\hline${ }^{11} \mathrm{~B}+{ }^{181} \mathrm{Ta}$ & 245.4 & $0.018 \pm 0.003$ & 0.020 \\
$p+{ }^{181} \mathrm{Ta}$ & 245.4 & $0.0025[83]$ & - \\
${ }^{11} \mathrm{~B}+{ }^{181} \mathrm{Ta}$ & 125.7 & $0.0065 \pm 0.0010$ & 0.0060 \\
$p+{ }^{181} \mathrm{Ta}$ & 125.7 & - & - \\
${ }^{11} \mathrm{~B}+{ }^{197} \mathrm{Au}$ & 255.5 & $0.45 \pm 0.09$ & 0.38 \\
$p+{ }^{197} \mathrm{Au}$ & 255.5 & $0.025[83]$ & - \\
${ }^{11} \mathrm{~B}+{ }^{197} \mathrm{Au}$ & 137.5 & $0.36 \pm 0.07$ & 0.38 \\
$p+{ }^{197} \mathrm{Au}$ & 137.5 & $0.016[83]$ & - \\
${ }^{7} \mathrm{Li}+{ }^{208} \mathrm{~Pb}$ & 245 & $0.57 \pm 0.17$ & 0.31 \\
$p+{ }^{208} \mathrm{~Pb}$ & 245 & - & - \\
${ }^{11} \mathrm{~B}+{ }^{209} \mathrm{Bi}$ & 146.0 & $0.46 \pm 0.07$ & 0.39 \\
$p+{ }^{209} \mathrm{Bi}$ & 146.0 & $0.075[83]$ & - \\
\hline \hline
\end{tabular}




\subsubsection{Possible mechanisms to the formation of IMFs}

As described in Sect. 5.1, IMFs are produced by the emission of light elements through different processes during the nucleus-nucleus reaction. As can be observed in Figs. 5.1 and 5.3, there is a large range of light fragments with $A<40 \mathrm{u}$ that are not explained by CRISP, as well as part of the heavy fragments with $A \sim 140-170 \mathrm{u}$ for ${ }^{181} \mathrm{Ta}$ target and with $A \sim 170-200 \mathrm{u}$ for ${ }^{209} \mathrm{Bi}$ target. The discrepancies between data and calculations can have their origins in two points: (i) there are some missing channels in the CRISP model; (ii) the spallation process with high angular momentum is not correctly described by CRISP. We will see that these two factors shall contribute to the partial disagreement between calculation and experiment.

Initially, we observe the remarkable agreements in the fission fragment mass regions after the effects of angular momentum are included in the fission potential. Hence, we consider that the fission mechanism is correctly described in our model, as well as all the mechanisms happening before fission takes place, namely, the initial interaction between the nuclei and the intranuclear cascade. From here, we conclude that the disagreement observed in other regions of the fragment mass distribution is due to a bad description of the spallation mechanism or due to some mechanisms competing with spallation that is missing in our model. Our intention here is not to include the missing channel in our model, but rather try to evaluate what this mechanism could be.

We noticed some similarities between IMF and spallation regions and tried to give an explanation about the possible mechanisms that could lead to the formation of these light and heavy fragments. The idea is that there is a mechanism that competes with fission and spallation which can produce heavy fragments by emission of clusters with $A<40$ u. At this point, we can make further considerations about the possible mechanism that is producing the excess of light fragments clearly observed in the experimental data: we see that this mechanism does not interfere with fission, which is correctly reproduced, but competes with spallation. The possible explanation for this behavior is that fission will happen only in the first steps of the evaporation chain, while the hypothetical mechanism would be a decay channel that mostly occurs after several steps in the evaporation, when a few nucleons and alpha particles were already evaporated. This can be a consequence of the fact that the fission probability decreases fast as the nuclear mass decreases. Also, 
this mechanism is likely to be a binary process, with a light fragment with mass up to 40 $\mathrm{u}$ being emitted and leaving a heavier counterpart with mass in the range between that of fission fragments and that of spallation.

Two possible hypothesis can be drawn from the above considerations: either most of the excitation energy is spent in the emission of the light fragment in the binary process; or the large fragment still has enough energy to evaporate more nucleons. Let us suppose that this process leads to the formation of two cold fragments, so no more nucleons can be emitted. In this case, being $A_{l}$ and $A_{h}$ the mass numbers of the light and heavy fragments, respectively, the distribution of the light fragment mass is related to the distribution of the heavy fragment, since their masses are related by $A_{l}+A_{h}=A_{0}$, where $A_{0}$ is the mass of the nucleus that is undergoing the hypothetical binary process. We will assume that the distribution of the light fragment mass is given by the Gaussian function:

$$
P\left(A_{l}\right)=p_{0} \exp \left[-0.5\left(\frac{A_{l}-p_{1}}{p_{2}}\right)^{2}\right]
$$

where $p_{0}, p_{1}$ and $p_{2}$ are fitting parameters shown in Table 5.9. The distribution of the heavy fragment will be, therefore, given by $P\left(A_{0}-A_{l}\right)$, where $A_{0}$ is to be determined by the following method: we observe that for small values of $A_{l}$, the mass of the heavy fragment is close to $A_{0}$ and therefore is in the region where the spallation peak gives a significant contribution to the total fragment mass distribution. On the other hand, we know from the considerations made above that $A_{0}$ should be somewhat smaller than the spallation peak, in order to not interfere significatively with the fission process. Therefore, we adjust the parameter $A_{0}$ in order to best fit the mass region of the smaller heavy fragments produced through the hypothetical mechanism, that is, the mass range just above the fission fragment mass region.

Since the binary process should compete with spallation, the total fragment mass distribution is obtained by summing the contribution from this mechanism to the spallation distribution. The results are presented in Fig. 5.5 and show that the introduction of this hypothetical mechanism could, indeed, improve the results, as the light fragment region can be accurately described and the mass region between the fission and spallation regions has also improved. While applying this mechanism for the reaction of ${ }^{11} \mathrm{~B}$ ion with ${ }^{209} \mathrm{Bi}$ at $146 \mathrm{MeV}$ results in a fairly good agreement between the CRISP and experimental data, 
Table 5.9: Parameters used to determine the distribution of the light fragment mass.

\begin{tabular}{|c|c|c|c|}
\hline \multirow[t]{2}{*}{ Parameter } & $\begin{array}{c}{ }^{11} B+{ }^{181} \mathrm{Ta} \\
(245.4 \mathrm{MeV})\end{array}$ & $\begin{array}{c}{ }^{11} B+{ }^{181} \mathrm{Ta} \\
(125.7 \mathrm{MeV})\end{array}$ & $\begin{array}{c}{ }^{11} B+{ }^{209} B i \\
(146 \mathrm{MeV})\end{array}$ \\
\hline & Value & Value & Value \\
\hline$p_{0}$ & 3.99 & 2.66 & 25.9 \\
\hline$p_{1}$ & 4.34 & 5.65 & -1.12 \\
\hline$p_{2}$ & 10.65 & 9.85 & 13.1 \\
\hline$A_{0}$ & 176 & 173 & 204 \\
\hline
\end{tabular}

some disagreement still persists in the region close to the spallation peak for the reactions of ${ }^{11} \mathrm{~B}+{ }^{181} \mathrm{Ta}$ at 245.4 and $125.7 \mathrm{MeV}$ energies, and it can indicate that the spallation process is not described in our model with the necessary accuracy. The CRISP model can give accurate results for spallation induced by photons, electrons or protons [32, 35, 84], but it was not tested before for the case of nucleus-nucleus reactions, when the high angular momentum can introduce important effects, as we observed in the case of fission. Therefore, we can suppose that, in the same way that the introduction of angular momentum dependency of the fission barrier is fundamental to obtain accurate results for fission cross section, similarly the effects of angular momentum in the evaporation of neutrons, protons and alpha particles may be relevant. If these effects enlarge the spallation peak at the left side which corresponds to lighter spallation products, the agreement with experiment could be improved. We will investigate this possibility, as well as the possible additional binary mechanism, in a future work. 


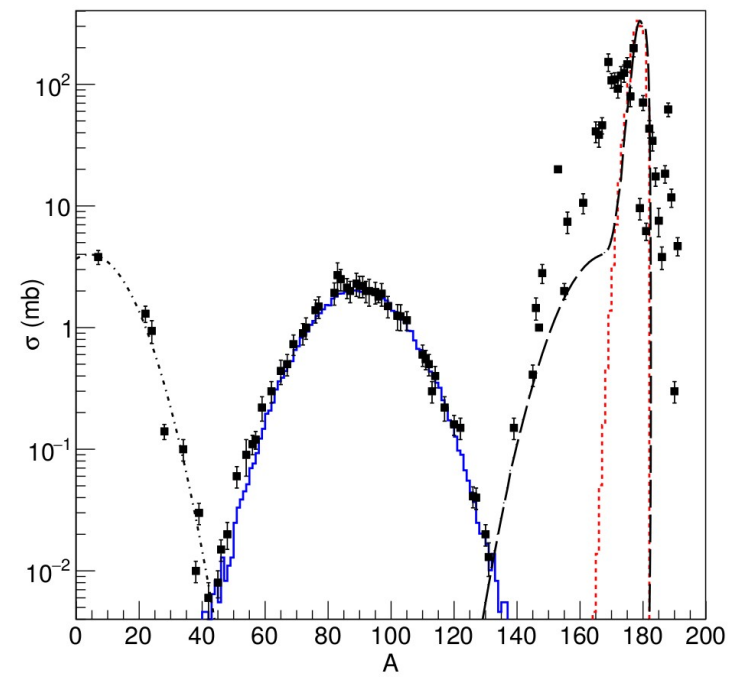

(a) ${ }^{181} \mathrm{Ta}+245.4 \mathrm{MeV}{ }^{11} \mathrm{~B}$

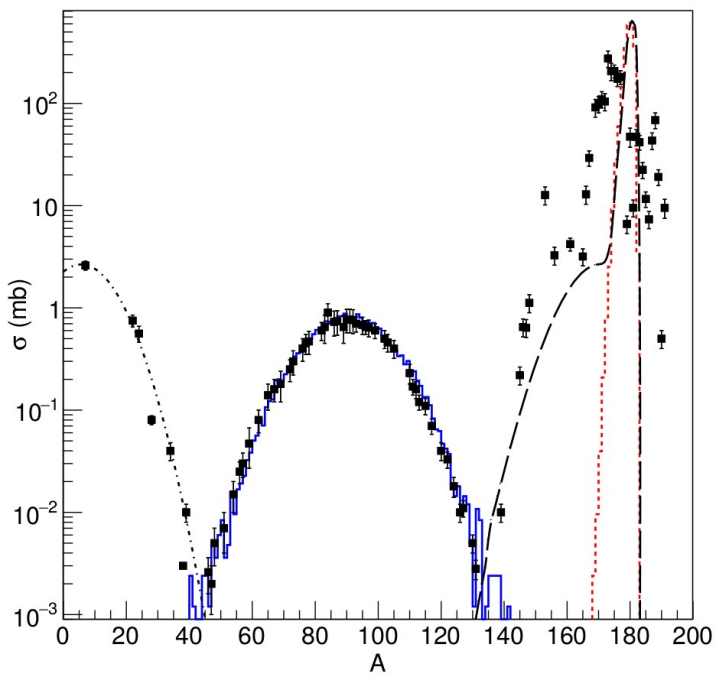

(b) ${ }^{181} \mathrm{Ta}+125.7 \mathrm{MeV}{ }^{11} \mathrm{~B}$

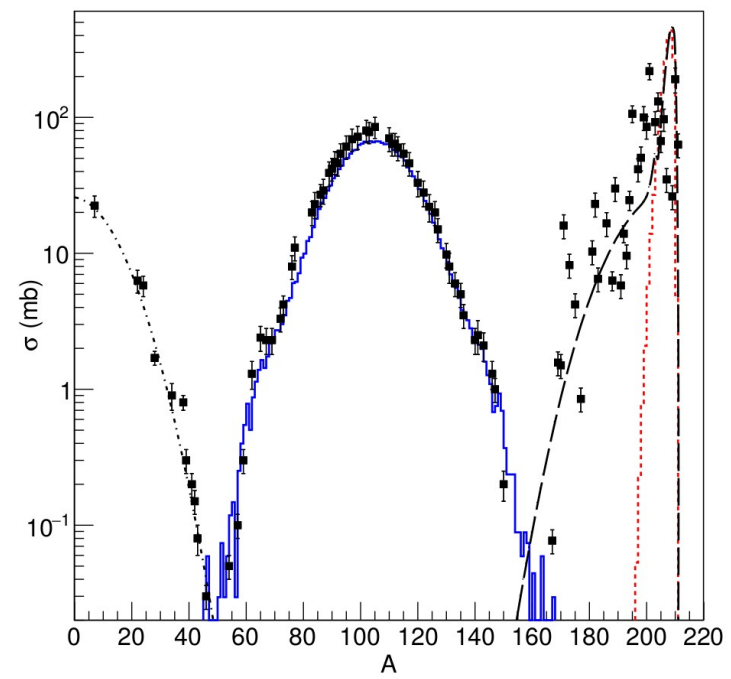

(c) ${ }^{209} \mathrm{Bi}+146 \mathrm{MeV}{ }^{11} \mathrm{~B}$

Figure 5.5: The same as the right column of Figs. 5.1 and 5.3. The black dash-dotted line corresponds to the results of the Gaussian fit to the IMF experimental data and the black dashed line is obtained by summing contribution from this mechanism to spallation distribution and indicates the addition of IMF cross section to the counterpart pair of products in spallation region. 


\section{Chapter 6}

\section{Conclusion}

In this work, a systematic analysis of the fragment mass distributions is performed by comparing the results of a developed branch of Monte Carlo CRISP model for nucleusnucleus reactions extended in the framework of the rotating liquid drop model. Since in heavy-ion induced reactions, a significant amount of angular momentum is transferred to the system, compared to the nucleon-nucleus and photonuclear collisions, fissionbarrier heights and consequently fission cross sections are calculated by implementation of RFRM and Nix model.

A qualitative analysis of fissioning systems is done by estimating the probability of different fission modes using the $Z_{f}^{2} / A_{f}$ criterion proposed by Chung et al. [81, 82]. The contribution of fissioning systems above and below the critical fissility parameter, and the pre- and post-scission emitted nucleons are described well using CRISP model.

Furthermore, heavy-ion induced fission, mass yield distribution of fission fragments and the effects associated with transferred angular momentum such as the angular momentum dependent fission barrier height and the fissility of the fissioning nuclei are well described by the new extended CRISP model. In conclusion, the results obtained after the implementation of rotating finite range model into the CRISP code are in good agreement with experimental data.

Besides, a possible mechanism is proposed for the formation of IMFs and the mass fragments between the fission and spallation regions. It is seen that the introduced hypothetical mechanism is able to describe the aformentioned regions fairly well. This work has opened the possibility to further studies on the new proposed mechanism and on the 
spallation by including angular momentum effects in evaporation of nucleons.

Just as fission-barrier heights are necessary for the calculation of fission and spallation cross sections, so are moments of inertia necessary for calculations of fission-fragment angular distributions. To calculate the three principal-axis moments of inertia of the saddle-point shape of a nucleus with given values of $Z, A$, and $L$ using RFRM model, a FORTRAN 77 computer subroutine called MOMFIT is written by Sierk [55]. To be able to use this code in CRISP, I have converted it from Fortran 77 into $\mathrm{C}++$ language. The next step in the future study can be the implementation of MOMFIT code into CRISP model to be able to calculate the rotational energy of the compound nucleus and describe the heavy-ion reaction better. 


\section{Chapter 7}

\section{Appendix}

\subsection{Appendix A}

Converted BARFIT subroutine into C++ language and implemented into CRISP code is brought here. 
1 \#include <iostream>

2 \#include <cstdlib>// for exit()

3 \#include <math. $h>$

4 using namespace std;

5 using std: :cerr;

6

7 int $\mathrm{A} ; / /$ mass number

8 int $\mathrm{Z} ; / /$ atomic number

9 double l; // angular momentum.

10 int $\mathrm{i}, \mathrm{j}, \mathrm{k}, \mathrm{m}, \mathrm{n} ; \mathrm{/}$ variables for loops

11

12 double bfis, bfise, egs, segs:

14 double aa, zz, a1, a2, aj, ak, amax, amax2, amin, amin2, q, qa, qb, x, y, z;

15 double aa, zz, al, a2, aj, ak, amax, amax2, amin, amin2,

16

18 double pl[10], pz[10], pa[10]

19

20 double* lpoly(double $\mathrm{x}$, int $\mathrm{n})\{/ /$ To return an array from function, it must be defined as a pointer.

22 static double temp[10]; // We define static to have a specific location in memory for array temp[10].

$23 \quad$ temp $[0]=1.0$;

$25 \operatorname{temp}[1]=\mathrm{x}$;

27 for (int $i=2 ; i<n ; i++)\{$

28 int $i \mathrm{i}=\mathrm{i}+1$

30

31

$\begin{array}{ll}33 & 3 \\ 34 & 3\end{array}$

35 return temp

$\left.\begin{array}{l}36 \\ 37\end{array}\right\}$

38

39 int main(int argc, char ** argv) \{

40

//double $2 \times 2$ = AngularMomentum().X()*AngularMomentum().X();

$42 \quad / /$ double $2 y 2=$ AngularMomentum(), $Y() *$ AngularMomentum(), $Y()$

$43 \quad / /$ double $l z 2$ = AngularMomentum(), $Z$ ()*AngularMomentum().Z();

$45 \quad / / 2=\operatorname{sqrt}(2 \times 2+l y 2+l z 2)$;

46 const double egsi[5][7] $=\{$

$\{-1.781665232 \mathrm{e} 6,4.358113622 \mathrm{e} 6,-4.804291019 \mathrm{e} 6,3.505397297 \mathrm{e} 6,-1.740990985 \mathrm{e} 6,5.492532874 \mathrm{e} 5,-9.229576432 \mathrm{e} 4\}$

$\{-2.849020290 \mathrm{e} 6,6.960182192 \mathrm{e} 6,-7.666333374 \mathrm{e} 6,5.586825123 \mathrm{e} 6,-2.759325148 \mathrm{e} 6,8.598827288 \mathrm{e} 5,-1.431344258 \mathrm{e} 5\}$

$\{9.546305856 \mathrm{e} 5,-2.381941132 \mathrm{e} 6,2.699742775 \mathrm{e} 6,-2.024820713 \mathrm{e} 6,1.036253535 \mathrm{e} 6,-3.399809581 \mathrm{e} 5,5.896521547 \mathrm{e} 4\}$,

$\{2.453904278 \mathrm{e} 5,-6.262569370 \mathrm{e} 5,7.415602390 \mathrm{e} 5,-5.818008462 \mathrm{e} 5,3.035749715 \mathrm{e} 5,-9.852362945 \mathrm{e} 4,1.772385043 \mathrm{e} 4\}$, \} ;

$\{3.656148926 \mathrm{e} 5,-9.026606463 \mathrm{e} 5,1.006008724 \mathrm{e} 6,-7.353683218 \mathrm{e} 5,3.606919356 \mathrm{e} 5,-1.108872347 \mathrm{e} 5,1.845424227 \mathrm{e}\}$,

const double egs2[5][7] $=\{$

$\{4.679351387 \mathrm{e} 6,-1.137635233 \mathrm{e} 7,1.237627138 \mathrm{e} 7,-8.854155353 \mathrm{e} 6,4.290642787 \mathrm{e} 6,-1.314924218 \mathrm{e} 6,2.131536582 \mathrm{e} 5\}$,

$\{7.707630513 \mathrm{e} 6,-1.870617878 \mathrm{e} 7,2.030222826 \mathrm{e} 7,-1.446966194 \mathrm{e} 7,6.951223648 \mathrm{e} 6,-2.095971932 \mathrm{e} 6,3.342907992 \mathrm{e}\}$,

$\{-2.718115276 \mathrm{e} 6,6.669154225 \mathrm{e} 6,-7.334289876 \mathrm{e} 6,5.295832834 \mathrm{e} 6,-2.601557110 \mathrm{e} 6,8.193066795 \mathrm{e} 5,-1.365390745 \mathrm{e} 5\}$

$\{-9.845252314 \mathrm{e} 5,2.413451470 \mathrm{e} 6,-2.656357635 \mathrm{e} 6,1.909275233 \mathrm{e} 6,-9.129731614 \mathrm{e} 5,2.716279969 \mathrm{e} 5,-4.417841315 \mathrm{e} 4\}$, \} ;

$\{-1.107173456 \mathrm{ee}, 2.691480439 \mathrm{e} 6,-2.912593917 \mathrm{e} 6,2.048899787 \mathrm{e} 6,-9.627344865 \mathrm{e} 5,2.823297853 \mathrm{e} 5,-4.427025540 \mathrm{e} 4\}$,

const double egs3[5][7] $=\{$

$\{-3.600471364 \mathrm{e} 6,8.829126250 \mathrm{e} 6,-9.781712604 \mathrm{e} 6,7.182555931 \mathrm{e} 6,-3.579820035 \mathrm{ee}, 1.122573403 \mathrm{e} 6,-1.839672155 \mathrm{e} 5\}$

$\{-5.805932202 \mathrm{e} 6,1.422377198 \mathrm{e} 7,-1.575666314 \mathrm{e} 7,1.156915972 \mathrm{e} 7,-5.740079339 \mathrm{e} 6,1.777161418 \mathrm{e} 6,-2.871137706 \mathrm{e} 5\}$

$\{1.773029253 \mathrm{e} 6,-4.473342834 \mathrm{e} 6,5.161226883 \mathrm{e} 6,-3.941330542 \mathrm{e} 6,2.041827680 \mathrm{e} 6,-6.714631146 \mathrm{e} 5,1.153532734 \mathrm{e} 5\}$,

$\{4.064280430 \mathrm{e} 5,-1.073350611 \mathrm{e} 6,1.341287330 \mathrm{e} 6,-1.108259560 \mathrm{e} 6,5.981648181 \mathrm{e} 5,-1.952833263 \mathrm{e} 5,3.423868607 \mathrm{e} 4\}$, \} ;

$\{7.419581557 \mathrm{e} 5,-1.845960521 \mathrm{e} 6,2.083994843 \mathrm{e} 6,-1.543982755 \mathrm{e} 6,7.629263278 \mathrm{e} 5,-2.328129775 \mathrm{e} 5,3.738902942 \mathrm{e} 4\}$,

const double egs4[5][7] $=\{$

$\{2.421750735 \mathrm{e} 6,-5.883394376 \mathrm{e} 6,6.387411818 \mathrm{e} 6,-4.550695232 \mathrm{e} 6,2.182540324 \mathrm{e} 6,-6.518758807 \mathrm{e} 5,9.952777968 \mathrm{e} 4\}$,

$\{4.107929841 \mathrm{e} 6,-9.964568970 \mathrm{e} 6,1.079547152 \mathrm{e} 7,-7.665548805 \mathrm{e} 6,3.646532772 \mathrm{e} 6,-1.070414288 \mathrm{e} 6,1.594230613 \mathrm{e} 5\}$,

$\{-1.302310290 \mathrm{e} 6,3.198405768 \mathrm{e} 6,-3.517981421 \mathrm{e} 6,2.530844204 \mathrm{e} 6,-1.228378318 \mathrm{e} 6,3.772592079 \mathrm{e} 5,-6.029082719 \mathrm{e} 4\}$

$\{-5.267906237 \mathrm{e}, 1.293156541 \mathrm{e} 6,-1.424705631 \mathrm{e}, 1.021187317 \mathrm{e},-4.813626449 \mathrm{e} 5,1.372024952 \mathrm{e} 5,-2.023689807 \mathrm{e} 4\}$, \} ;

$\{-6.197966854 \mathrm{e} 5,1.506909314 \mathrm{e} 6,-1.629099740 \mathrm{e} 6,1.141553709 \mathrm{e} 6,-5.299974544 \mathrm{e} 5,1.505359294 \mathrm{e} 5,-2.176008230 \mathrm{e} 4\}$

const double egs5[5][7] $=\{$

$\{-4.902668827 \mathrm{e} 5,1.231673941 \mathrm{e} 6,-1.429330809 \mathrm{e} 6,1.114306796 \mathrm{e} 6,-5.873353309 \mathrm{e} 5,1.895325584 \mathrm{e} 5,-2.969272274 \mathrm{e} 4\}$,

$\{-8.089034293 \mathrm{e} 5,2.035989814 \mathrm{e} 6,-2.376692769 \mathrm{e} 6,1.868800148 \mathrm{e} 6,-9.903614817 \mathrm{e} 5,3.184776808 \mathrm{e} 5,-4.916872669 \mathrm{e} 4\}$

$\{1.282510910 \mathrm{e} 5,-3.727491110 \mathrm{e} 5,5.216954243 \mathrm{e} 5,-4.718718351 \mathrm{e} 5,2.742543392 \mathrm{e} 5,-9.500485442 \mathrm{e} 4,1.596305804 \mathrm{e} 4\}$,

$\{-1.704435174 \mathrm{e} 4,4.071377327 \mathrm{e} 3,7.268703575 \mathrm{e} 4,-1.215904582 \mathrm{e} 5,9.055579135 \mathrm{e} 4,-3.406036086 \mathrm{e} 4,5.741228836 \mathrm{e}\}\}$,

\};

$\{8.876109934 \mathrm{e} 4,-2.375344759 \mathrm{e} 5,3.008350125 \mathrm{e} 5,-2.510379590 \mathrm{e} 5,1.364869036 \mathrm{e} 5,-4.380685984 \mathrm{e} 4,6.669912421 \mathrm{e}\}$,

// cout $\ll$ "egs1: " $<$ egsI[0][0] $\ll$ endl;

$/ /$ const double egscof[5][7][5] $=\{$

// $\{$ egs1[5][7], egs2[5][7], egs3[5][7], egs4[5][7], egs5[5][7]\}

$/ /\}$

double egscof[5][7][5];

for (int $i=0 ; i<5$; ++i)

\{

for (int $j=0 ; j<7 ;++j$ )

\}

$\operatorname{egscof}[i][j][0]=\operatorname{egs} 1[i][j] ;$

for (int $i=0 ; i<5 ;++i)$

for (int $j=0 ; j<7 ;++j$ )

\}

$\operatorname{egscof}[i][j][1]=\operatorname{egs} 2[i][j] ;$

for (int $i=0 ; i<5 ;++i)$ 


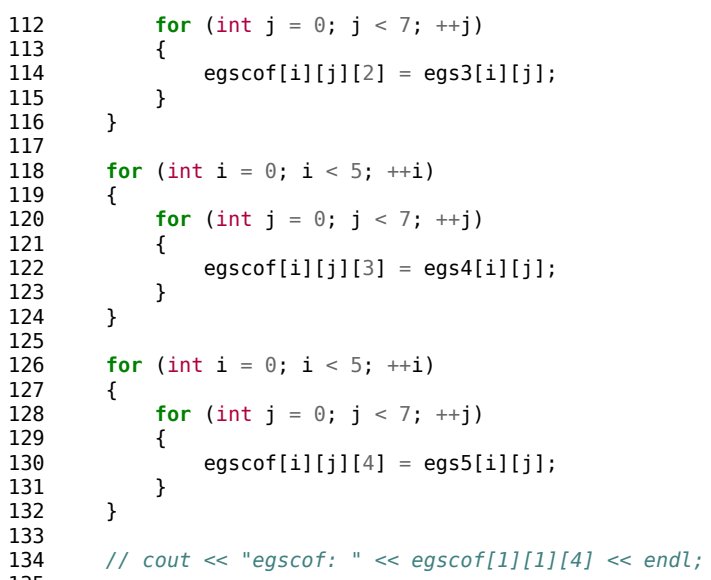

const double emncof[5][4] $=\{$

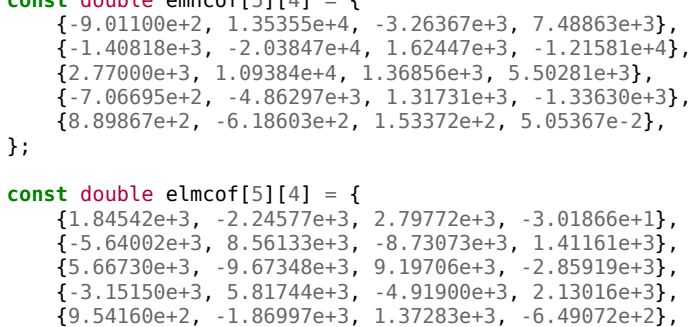

$\{-4.10652732 \mathrm{e} 6,1.08763330 \mathrm{e} 7,-8.76530903 \mathrm{e} 6,6.30258954 \mathrm{e} 6,-1.45539891 \mathrm{e} 6\}$,

$\{1.00064947 \mathrm{e} 7,-2.63758245 \mathrm{e} 7,2.14250513 \mathrm{e} 7,-1.52999004 \mathrm{e} 7,3.64961835 \mathrm{e} 6\}$,

$\{-1.09533751 \mathrm{e} 7,2.85472400 \mathrm{e} 7,-2.35799595 \mathrm{e}, 1.65640200 \mathrm{e} 7,-4.21267423 \mathrm{e} 6\}$

$\{7.84797252 \mathrm{e} 6,-2.01107467 \mathrm{e} 7,1.70161347 \mathrm{e} 7,-1.16695776 \mathrm{e} 7,3.24312555 \mathrm{e} 6\}$,

$\{-3.78574926 \mathrm{e} 6,9.48373641 \mathrm{e} 6,-8.23738190 \mathrm{e} 6,5.47369153 \mathrm{e} 6,-1.67927904 \mathrm{e} 6\}$

$\{1.12237945 \mathrm{e} 6,-2.73438528 \mathrm{e} 6,2.42447957 \mathrm{e} 6,-1.54986342 \mathrm{e} 6,-5.23795062 \mathrm{e} 5\}$, \};

$\{-1.77561170 \mathrm{e} 5,4.13247256 \mathrm{ee},-3.65427239 \mathrm{e} 5,2.15409246 \mathrm{e} 5,-7.66576599 \mathrm{e} 4\}$,

const double elzcof[7][7] $=\{$

$\{5.11819909 \mathrm{e}+5,-1.13269453 \mathrm{e}+6,1.37543304 \mathrm{e}+6,-8.56559835 \mathrm{e}+5,3.28723311 \mathrm{e}+5,4.15850238 \mathrm{e}+4,-1.82751044 \mathrm{e}+5\}$,

$\{-1.30303186 e+6,2.97764590 e+6,-3.65808988 e+6,2.48872266 e+6,-1.09892175 e+6,7.29653408 e+4,3.91386300 e+5\}$,

$\{1.90119870 \mathrm{e}+6,-4.54326326 \mathrm{e}+6,5.47798999 \mathrm{e}+6,-4.07349128 \mathrm{e}+6,2.03997269 \mathrm{e}+6,-4.93776346 \mathrm{e}+5,-3.03639248 \mathrm{e}+5\}$,

$\{-1.20628242 \mathrm{e}+6,3.00464870 \mathrm{e}+6,-3.78109283 \mathrm{e}+6,3.12835899 \mathrm{e}+6,-1.77185718 \mathrm{e}+6,6.01254680 \mathrm{e}+5,1.15782417 \mathrm{e}+5\}$,

$\{5.68208488 \mathrm{e}+5,-1.44989274 \mathrm{e}+6,1.84131765 \mathrm{e}+6,-1.62394090 \mathrm{e}+6,9.96051545 \mathrm{e}+5,-4.01308292 \mathrm{e}+5,-4.24399280 \mathrm{e}+3\}$

$\{5.48346483 e+4,-1.02026610 e+5,1.53669695 e+4,1.19797378 e+5,-1.53305699 e+5,9.65968391 e+4,-6.11477247 e+3\}$,

\} ;

$\{-2.45883052 \mathrm{e}+4,6.27959815 \mathrm{e}+4,-6.96817834 \mathrm{e}+4,4.25737058 \mathrm{e}+4,-1.12982954 \mathrm{e}+4,-3.49596027 \mathrm{e}+3,3.66982647 \mathrm{e}+2\}$

$\mathrm{Z}=65$

$l=50$

if $(Z<19$ || $Z>111)\{$

segs $=0.0$;

selmax $=0.0 ;$

std: : cout $\ll " * * * *$ barfit is called with $\mathrm{Z}$ less than 19 or greater than 111 . bfis is set to 0.0 . $* * * * \|<$ endl

\}

return bfis, segs, selmax;

if $(Z>102 \& \& \quad l>0)\{$

bfis $=0.0$

selmax $=0.0$;

std: : cout $<" * * * *$ barfit is called with Z greater than 102 and $\mathrm{L}$ not equal to zero. bfis is set to 0.0 . $* * * * "<$ end

\}

return bfis, segs, selmax

$A=153 ;$
el $=1 ;$

amin $=1.2 * Z+0.01 * Z * Z$;

$\operatorname{amax}=5.8 * Z-0.024 * Z * Z$;

if $(A<\operatorname{amin}|| A>\operatorname{amax})\{$

bfis $=0.0$

selmax $=0.0$

std: : cout $\ll " * * * *$ barfit is called with A outside the allowed values $* * * * 1<$ endl;

return bfis, segs, selmax;

$\mathrm{aa}=\mathrm{A} / 400.0$;

$\mathrm{ZZ}=\mathrm{Z} / 100.0$

bfis $\theta=0.0$

$/ /$ double $* p l=$ lpoly $(0.28,3): / / p l$ is defined as a pointer

//Since the function lpoly returns an array or a pointer, we can assign lpoly to the pointer pl.

// cout $\ll p l[1] \ll$ endl;

// cout $\ll *(p l+1) \ll$ endl; // another way to return the arrays.

double $*$ temppz $=\operatorname{lpoly}(z z, 7)$

for (int $i=0 ; i<7 ; i++$ ) 


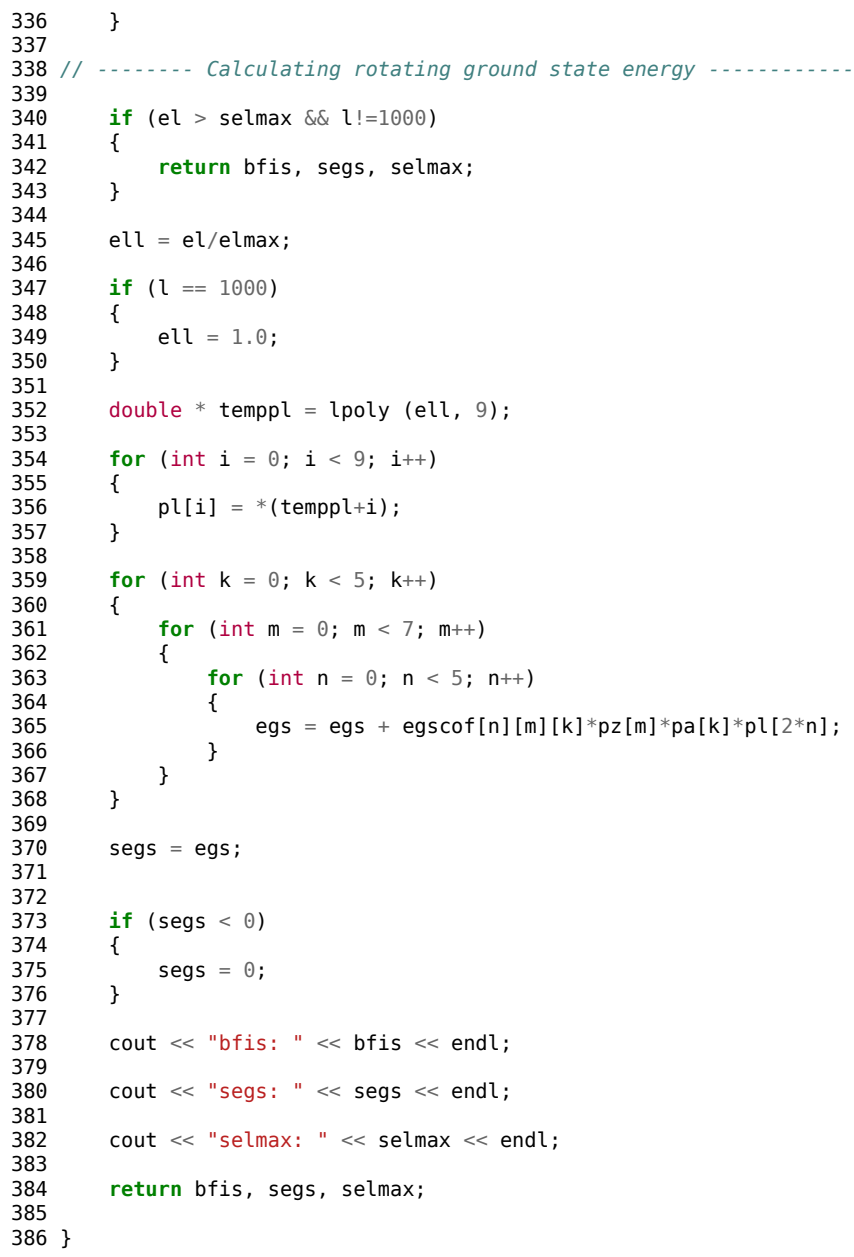




\section{Bibliography}

[1] G. S. Karapetyan, A. Deppman, V. Guimarães, A. Balabekyan, and N. A. Demekhina. Interaction of ${ }^{11} \mathrm{~B}$ with ${ }^{209} \mathrm{Bi}$ and ${ }^{181} \mathrm{Ta}$ targets at intermediate energies. Phys. Rev. C, 94:024618, Aug 2016.

[2] Robert Vandenbosch and John R Huizenga. Nuclear Fission. Academic Press., New York, 1973.

[3] D. Hilscher, I. I. Gontchar, and H. Rossner. Fission dynamics of hot nuclei and nuclear dissipation. Physics of Atomic Nuclei, 57:1187-1199, July 1994.

[4] Wikipedia contributors. Nuclear fission - Wikipedia, the free encyclopedia, 2020. [Online; accessed 6-September-2020].

[5] A Deppman, S B Duarte, G Silva, O A P Tavares, S Anéfalos, J D T Arruda-Neto, and $\mathrm{T}$ E Rodrigues. The crisp package for intermediate- and high-energy photonuclear reactions. Journal of Physics G: Nuclear and Particle Physics, 30(12):1991, 2004.

[6] Davide Mancusi, Alain Boudard, Joseph Cugnon, Jean-Christophe David, Pekka Kaitaniemi, and Sylvie Leray. Extension of the liege intranuclear-cascade model to reactions induced by light nuclei. Phys. Rev. C, 90:054602, Nov 2014.

[7] A. Boudard, J. Cugnon, J.-C. David, S. Leray, and D. Mancusi. New potentialities of the liège intranuclear cascade model for reactions induced by nucleons and light charged particles. Phys. Rev. C, 87:014606, Jan 2013.

[8] L. Audirac, A. Obertelli, P. Doornenbal, D. Mancusi, S. Takeuchi, N. Aoi, H. Baba, S. Boissinot, A. Boudard, A. Corsi, A. Gillibert, T. Isobe, A. Jungclaus, V. Lapoux, 
J. Lee, S. Leray, K. Matsui, M. Matsushita, T. Motobayashi, D. Nishimura, S. Ota, E. C. Pollacco, G. Potel, H. Sakurai, C. Santamaria, Y. Shiga, D. Sohler, D. Steppenbeck, R. Taniuchi, and H. Wang. Evaporation-cost dependence in heavy-ion fragmentation. Phys. Rev. C, 88:041602, Oct 2013.

[9] S Leray, D Mancusi, P Kaitaniemi, J C David, A Boudard, B Braunn, and J Cugnon. Extension of the liège intra nuclear cascade model to light ion-induced collisions for medical and space applications. Journal of Physics: Conference Series, 420:012065, mar 2013.

[10] A. Boudard, J. Cugnon, S. Leray, and C. Volant. Intranuclear cascade model for a comprehensive description of spallation reaction data. Phys. Rev. C, 66:044615, Oct 2002.

[11] K. Mahata, S. Kailas, and S. S. Kapoor. Fission barrier, damping of shell correction, and neutron emission in the fission of $a \sim 200$. Phys. Rev. C, 92:034602, Sep 2015.

[12] Ernest M. Henley and Alejandro Garcia. Subatomic Physics. World Scientific Publishing Co. Pte. Ltd., 3rd edition, 2007.

[13] Amos DeShalit and Herman Feshbach. Theoretical Nuclear Physics: Nuclear structure. Wiley, 1st edition, 1974.

[14] C. H. Townes, H. M. Foley, and W. Low. Nuclear quadrupole moments and nuclear shell structure. Phys. Rev., 76:1415-1416, Nov 1949.

[15] James Rainwater. Nuclear energy level argument for a spheroidal nuclear model. Phys. Rev., 79:432-434, Aug 1950.

[16] Aage Bohr. On the quantization of angular momenta in heavy nuclei. Phys. Rev., 81:134-138, Jan 1951.

[17] S. G. Nilsson. Binding states of individual nucleons in strongly deformed nuclei. Kgl. Danske Videnskab, Selskab. Mat.-fys. Medd., 29:1 - 69, 1955. 
[18] William D. Myers and Wladyslaw J. Swiatecki. Anomalies in nuclear masses. Lawrence Radiation Laboratory, University of California, Berkeley, California, 1966.

[19] William D. Myers and Wladyslaw J. Swiatecki. Nuclear masses and deformations. Nuclear Physics, 81(1):1 - 60, 1966.

[20] Ulrich Brosa, Siegfried Grossmann, and Andreas Müller. Nuclear scission. Physics Reports, 197(4):167 - 262, 1990.

[21] T. Kodama, S. B. Duarte, K. C. Chung, and R. A. M. S. Nazareth. Cluster approach to intranuclear cascade for relativistic heavy-ion collisions. Phys. Rev. Lett., 49:536539, Aug 1982.

[22] M. Gonçalves, S. de Pina, D.A. Lima, W. Milomen, E.L. Medeiros, and S.B. Duarte. Many-body cascade calculation for photonuclear reactions. Physics Letters $B, 406(1): 1-6,1997$.

[23] A. Deppman, O.A.P. Tavares, S.B. Duarte, E.C. de Oliveira, J.D.T. Arruda-Neto, S.R. de Pina, V.P. Likhachev, O. Rodriguez, J. Mesa, and M. Gonçalves. The mcef code for nuclear evaporation and fission calculations. Computer Physics Communications, 145(3):385 - 394, 2002.

[24] I Gonzalez, C Barbero, A Deppman, S B Duarte, F Krmpotić, and O Rodriguez. Many-body cascade calculation of final state interactions in ${ }_{\lambda}^{12} \mathrm{c}$ nonmesonic weak decay. Journal of Physics G: Nuclear and Particle Physics, 38(11):115105, 2011.

[25] A. Deppman, O.A.P. Tavares, S.B. Duarte, J.D.T. Arruda-Neto, M. Gonçalves, V.P. Likhachev, J. Mesa, E.C. de Oliveira, S.R. de Pina, and O. Rodriguez. A monte carlo method for nuclear evaporation and fission at intermediate energies. Nuclear Instruments and Methods in Physics Research Section B: Beam Interactions with Materials and Atoms, 211(1):15 - 21, 2003.

[26] E Andrade-II, J C M Menezes, S B Duarte, F Garcia, P C R Rossi, O A P Tavares, and A Deppman. Fragment mass distributions in the fission of heavy nuclei by 
intermediate- and high-energy probes. Journal of Physics G: Nuclear and Particle Physics, 38(8):085104, 2011.

[27] A. Deppman, O. A. P. Tavares, S. B. Duarte, E. C. de Oliveira, J. D. T. ArrudaNeto, S. R. de Pina, V. P. Likhachev, O. Rodriguez, J. Mesa, and M. Gonçalves. Photofissility of actinide nuclei at intermediate energies. Phys. Rev. Lett., 87:182701, Oct 2001 .

[28] A. Deppman, G. Silva, S. Anefalos, S. B. Duarte, F. García, F. H. Hisamoto, and O. A. P. Tavares. Photofission and total photoabsorption cross sections in the energy range of shadowing effects. Phys. Rev. C, 73:064607, Jun 2006.

[29] A. Deppman, O. A. P. Tavares, S. B. Duarte, J. D. T. Arruda-Neto, M. Gonçalves, V. P. Likhachev, and E. C. de Oliveira. Photofissility of heavy nuclei at intermediate energies. Phys. Rev. C, 66:067601, Dec 2002.

[30] E Andrade-II, J C M Menezes, S B Duarte, F Garcia, P C R Rossi, O A P Tavares, and A Deppman. Effects of fissioning nuclei distributions on fragment mass distributions for high energy fission. EPJ Web of Conferences, 21:10001, 2012.

[31] A. Deppman, E. Andrade-II, V. Guimarães, G. S. Karapetyan, A. R. Balabekyan, and N. A. Demekhina. Proton-induced fission on ${ }^{241} \mathrm{am},{ }^{238} \mathrm{u}$, and ${ }^{237} \mathrm{np}$ at intermediate energies. Phys. Rev. C, 88:024608, Aug 2013.

[32] A. Deppman, E. Andrade-II, V. Guimarães, G. S. Karapetyan, O. A. P. Tavares, A. R. Balabekyan, N. A. Demekhina, J. Adam, F. Garcia, and K. Katovsky. Superasymmetric fission of heavy nuclei induced by intermediate-energy protons. Phys. Rev. C, 88:064609, Dec 2013.

[33] E. Andrade-II, G. S. Karapetyan, A. Deppman, A. R. Balabekyan, N. A. Demekhina, and V. Guimarães. Photon and proton induced fission on heavy nuclei at intermediate energies. EPJ Web of Conferences, 69:00017, 2014.

[34] A. Deppman, G. S. Karapetyan, V. Guimarães, C. Gonzales, A. R. Balabekyan, and N. A. Demekhina. Bremsstrahlung-induced fission and spallation of the pre-actinide nucleus ${ }^{181}$ Ta. Phys. Rev. C, 91:024620, Feb 2015. 
[35] E Andrade-II, G S Karapetyan, A Deppman, J L Bernal-Castillo, Valdir Guimarães, A R Balabekyan, J Adam, F Garcia, and F Guzmán. Systematic study of fission modes in the proton-induced fission of actinide and pre-actinide. Journal of Physics G: Nuclear and Particle Physics, 45(1):015105, dec 2017.

[36] A. Deppman, E. Andrade-II, V. Guimarães, G. S. Karapetyan, and N. A. Demekhina. Photofission of ${ }^{232}$ th and ${ }^{238} \mathrm{u}$ at intermediate energies. Phys. Rev. C, 87:054604, May 2013.

[37] A. Deppman, E. Andrade-II, P. C. R. Rossi, F. Garcia, and J. R. Maiorino. Research article, monte carlo calculation of fragment distributions in nuclear reactions. Science and Technology of Nuclear Installations, 73:480343, March 2012.

[38] S. Anefalos, A. Deppman, Gilson da Silva, J. R. Maiorino, A. dos Santos, and F. Garcia. Development of the crisp package for spallation studies and accelerator-driven systems. Nuclear Science and Engineering, 151(1):82-87, 2005.

[39] S. Anefalos, A. Deppman, J. D. T. Arruda-Neto, V.P. Likhachev, A. dos Santos, P. R. P. Coelho, S. B. Duarte, and O. A. P. Tavares. Developement of crisp package for spallation reaction studies and its utilization in ads (accelerator driven system). Brazilian Journal of Physics, 34:966-968, 092004.

[40] S. Anefalos, A. Deppman, G. Silva, J. R. Maiorino, A. dos Santos, S. B. Duarte, O. A. P. Tavares, and F. Garcia. The utilization of crisp code in hybrid reactor studies. Brazilian Journal of Physics, 35:912 - 914, 092005.

[41] S. Anáfalos Pereira, A. Deppman, G. Silva, J. R. Maiorino, A. dos Santos, S. B. Duarte, O. A. P. Tavares, and F. Garcia. Spallation product distributions and neutron multiplicities for accelerator-driven system using the crisp code. Nuclear Science and Engineering, 159(1):102-105, 2008.

[42] V. Weisskopf. Statistics and nuclear reactions. Phys. Rev., 52:295-303, Aug 1937.

[43] Niels Bohr and John Archibald Wheeler. The mechanism of nuclear fission. Phys. Rev., 56:426-450, Sep 1939. 
[44] J. M. Pearson. The quest for a microscopic nuclear mass formula. Hyperfine Interactions, 132(1):59-74, Jan 2001.

[45] I. Dostrovsky, P. Rabinowitz, and R. Bivins. Monte carlo calculations of high-energy nuclear interactions. i. systematics of nuclear evaporation. Phys. Rev., 111:16591676, Sep 1958.

[46] O A P Tavares and M L Terranova. Analysis of ${ }^{209} \mathrm{Bi}$ and ${ }^{238} \mathrm{U}$ photofission cross section in the quasi-deuteron region of photonuclear absorption. Z. Physik A - Hadrons and Nuclei, 343:407-416, 1992.

[47] V.V. Pashkevich. On the asymmetric deformation of fissioning nuclei. Nuclear Physics A, 169(2):275 - 293, 1971.

[48] B. D. Wilkins, E. P. Steinberg, and R. R. Chasman. Scission-point model of nuclear fission based on deformed-shell effects. Phys. Rev. C, 14:1832-1863, Nov 1976.

[49] W. Younes, J. A. Becker, L. A. Bernstein, P. E. Garrett, C. A. McGrath, D. P. McNabb, R. O. Nelson, W. S. Johns, G. D. amd Wilburn, and D. M. Drake. Transition from asymmetric to symmetric fission in the $235 \mathrm{u}(\mathrm{n}, \mathrm{f})$ reaction. AIP Conference Proceedings, 610(1):673-677, 2002.

[50] N. A. Demekhina and G. S. Karapetyan. Multimode approximation for 238u photofission at intermediate energies. Physics of Atomic Nuclei, 71(1):27-35, Jan 2008.

[51] G. S. Karapetyan, A. R. Balabekyan, N. A. Demekhina, and J. Adam. Multimode approach to $241 \mathrm{am}$ and $237 \mathrm{np}$ fission induced by $660 \mathrm{-mev}$ protons. Physics of Atomic Nuclei, 72(6):911-916, Jun 2009.

[52] H. Kudo, M. Maruyama, M. Tanikawa, T. Shinozuka, and M. Fujioka. Most probable charge of fission products in 24 mev proton induced fission of ${ }^{238} \mathrm{U}$. Phys. Rev. C, 57:178-188, Jan 1998.

[53] M. C. Duijvestijn, A. J. Koning, J. P. M. Beijers, A. Ferrari, M. Gastal, J. van Klinken, and R. W. Ostendorf. Proton-induced fission at $190 \mathrm{mev}$ of ${ }^{\text {nat }} \mathrm{W},{ }^{197} \mathrm{Au}$, ${ }^{\text {nat }} \mathrm{Pb},{ }^{208} \mathrm{~Pb}$, and ${ }^{232}$ Th. Phys. Rev. C, 59:776-788, Feb 1999. 
[54] M. G. Mustafa, P. A. Baisden, and H. Chandra. Equilibrium shapes and fission barriers of rotating nuclei with a macroscopic two-center model. Phys. Rev. C, 25:25242533, May 1982.

[55] Arnold J. Sierk. Macroscopic model of rotating nuclei. Phys. Rev. C, 33:2039-2053, Jun 1986.

[56] M. Abbasi, H. Panjeh, R. Perez, A. Deppman, E. Andrade-II, F. Velasco, and F. Guzman. Heavy-ion-induced fission of ${ }^{181} \mathrm{Ta}$ and ${ }^{209} \mathrm{Bi}$ at intermediate energies by crisp model. Eur. Phys. J. Plus, 135(10):845, 2020.

[57] G. L. Kotkin and V. G. Serbo. Collection of Problems in Classical Mechanics. International Series of Monographs in Natural Philosophy. Pergamon Press, 1st edition, 1971.

[58] P. Frobrich and I.I. Gontchar. Langevin description of fusion, deep-inelastic collisions and heavy-ion-induced fission. Physics Reports, 292(3):131 - 237, 1998.

[59] J. Wilczyński. Calculations of the critical angular momentum in the entrance reaction channel. Nuclear Physics A, 216(2):386 - 394, 1973.

[60] O. A. Capurro, D. E. DiGregorio, S. Gil, D. Abriola, M. di Tada, J. O. Fernández Niello, A. O. Macchiavelli, G. V. Martí, A. J. Pacheco, J. E. Testoni, D. Tomasi, and I. Urteaga. Average angular momentum in compound nucleus reactions deduced from isomer ratio measurements. Phys. Rev. C, 55:766-774, Feb 1997.

[61] Stanley Cohen and W.J Swiatecki. The deformation energy of a charged drop: Iv. evidence for a discontinuity in the conventional family of saddle point shapes. Annals of Physics, 19(1):67 - 164, 1962.

[62] Stanley Cohen and Wladyslaw J Swiatecki. The deformation energy of a charged drop: Part v: Results of electronic computer studies. Annals of Physics, 22(3):406 437, 1963.

[63] G. A. PIK-PICHAK. Fission of rotating nuclei. Soviet Physics JETP, 7:238-241, Aug 1958. 
[64] G. A. PIK-PICHAK. Fission barrier of a rotating nucleus. Soviet Physics JETP, 15:897-902, November 1962.

[65] Robert Beringer and W. J. Knox. Liquid-drop nuclear model with high angular momentum. Phys. Rev., 121:1195-1200, Feb 1961.

[66] S. Cohen, F. Plasil, and W.J. Swiatecki. Equilibrium configurations of rotating charged or gravitating liquid masses with surface tension. ii. Annals of Physics, 82(2):557 - 596, 1974.

[67] M. Beckerman and M. Blann. Fission barriers at high angular momenta. Phys. Rev. Lett., 38:272-275, Feb 1977.

[68] F. Plasil, R. L. Ferguson, R. L. Hahn, Felix E. Obenshain, Frances Pleasonton, and G. R. Young. Fission barrier of ${ }^{153}$ Tb. Phys. Rev. Lett., 45:333-336, Aug 1980.

[69] D.J. Hinde, J.R. Leigh, J.O. Newton, W. Galster, and S. Sie. Fission and evaporation competition in 200pb. Nuclear Physics A, 385(1):109 - 132, 1982.

[70] D.J. Hinde, J.O. Newton, J.R. Leigh, and R.J. Charity. Fission barriers of pb nuclei at high angular momentum. Nuclear Physics A, 398(2):308 - 324, 1983.

[71] C. Cabot1, H. Gauvin, Y. Le Beyec, H. Delagrange, J.P. Dufour, A. Fleury, Y. Llabador, and J.M. Alexander. Evaporation residue and fission cross-section for the system : ${ }^{40} \mathrm{Ar}+{ }^{110} \mathrm{Pd}$. J. Phys. Colloques, 41(12):C10-234-C10-238, 1980.

[72] J. R. Leigh, D. J. Hinde, J. O. Newton, W. Galster, and S. H. Sie. Fission-imposed limits to the angular momentum carried by evaporation residues from the compound nucleus ${ }^{200}$ Pb. Phys. Rev. Lett., 48:527-530, Feb 1982.

[73] B. Sikora, W. Scobel, M. Beckerman, J. Bisplinghoff, and M. Blann. Measurement and statistical analyses of fission and fusion excitation functions for ${ }^{35} \mathrm{Cl}$ on ${ }^{62} \mathrm{Ni}$, ${ }^{116} \mathrm{Sn}$, and ${ }^{141} \mathrm{Pr}$ up to $E_{\text {lab }}=215$ mev. Phys. Rev. C, 25:1446-1459, Mar 1982.

[74] G. Guillaume, J. P. Coffin, F. Rami, P. Engelstein, B. Heusch, P. Wagner, P. Fintz, J. Barrette, and H. E. Wegner. Fissionlike component in the ${ }^{32} \mathrm{~S}+{ }^{76} \mathrm{Ge}$ reaction. Phys. Rev. C, 26:2458-2469, Dec 1982. 
[75] Hiroshi Baba, Atsushi Shinohara, Tadashi Saito, Naruto Takahashi, and Akihiko Yokoyama. Specific fission j-window and angular momentum dependence of the fission barrier. Journal of the Physical Society of Japan, 66(4):998-1009, 1997.

[76] H. J. Krappe, J. R. Nix, and A. J. Sierk. Unified nuclear potential for heavy-ion elastic scattering, fusion, fission, and ground-state masses and deformations. Phys. Rev. C, 20:992-1013, Sep 1979.

[77] M. Blann. Observations on rotating finite-range fission barriers derived from barriers for nonrotating nuclei. Phys. Rev. C, 34:2215-2216, Dec 1986.

[78] Nicolae Carjan and Morton Kaplan. Asymmetric fission of ${ }^{149} \mathrm{~Tb}^{*}$ from the finiterange, rotating-liquid-drop model: Mean total kinetic energies for binary fragmentation. Phys. Rev. C, 45:2185-2195, May 1992.

[79] James Rayford Nix. Further studies in the liquid-drop theory on nuclear fission. Nuclear Physics A, 130(2):241 - 292, 1969.

[80] Y. Yariv and Z. Fraenkel. Intranuclear cascade calculation of high-energy heavy-ion interactions. Phys. Rev. C, 20:2227-2243, Dec 1979.

[81] Chien Chung and James J. Hogan. Fission of ${ }^{232}$ Th at energies up to 90 mev. Phys. Rev. C, 24:180-191, Jul 1981.

[82] Chien Chung and James J. Hogan. ${ }^{238} \mathrm{U}$ fission at energies up to 100 mev. Phys. Rev. C, 25:899-908, Feb 1982.

[83] Tokio Fukahori, Osamu Iwamoto, and Satoshi Chiba. Unified description of neutron-, proton- and photon-induced fission cross sections in intermediate energy region. Proceedings of the Seventh International Conference on Nuclear Criticality Safety, ICNC2003, JAERI-conf, 2003-019-PT2, Japan, 36(50):603-608, 2003.

[84] A. R. Balabekyan, G. S. Karapetyan, E. Andrade-II, A. Deppman, S. V. Gaginyan, E. Melyan, J. R. Drnoyan, V. I. Zhemenik, J. Adam, L. Zavorka, A. A. Solnyshkin, V. M. Tsoupko-Sitnikov, J. Khushvaktov, and E. B. Hernández. Fragment production in proton-induced reaction on ${ }^{209} \mathrm{Bi}$ target at intermediate energy range. Phys. Rev. C, 100:024616, Aug 2019. 